\title{
INFERENCE ON DISTRIBUTION FUNCTIONS UNDER MEASUREMENT ERROR
}

\author{
KARUN ADUSUMILLI, DAISUKE KURISU, TAISUKE OTSU, AND YOON-JAE WHANG
}

ABstract. This paper is concerned with inference on the cumulative distribution function (cdf) $F_{X^{*}}$ in the classical measurement error model $X=X^{*}+\epsilon$. We consider the case where the density of the measurement error $\epsilon$ is unknown and estimated by repeated measurements, and show validity of a bootstrap approximation for the distribution of the deviation in the sup-norm between the deconvolution cdf estimator and $F_{X^{*}}$. We allow the density of $\epsilon$ to be ordinary or super smooth. We also provide several theoretical results on the bootstrap and asymptotic Gumbel approximations of the sup-norm deviation for the case where the density of $\epsilon$ is known. Our approximation results are applicable to various contexts, such as confidence bands for $F_{X^{*}}$ and its quantiles, and for performing various cdf-based tests such as goodness-of-fit tests for parametric models of $X^{*}$, two sample homogeneity tests, and tests for stochastic dominance. Simulation and real data examples illustrate satisfactory performance of the proposed methods.

\section{INTRODUCTION}

This paper is concerned with inference on the cumulative distribution function (cdf) $F_{X^{*}}$ in the classical measurement error model $X=X^{*}+\epsilon$. Here, we observe $X$ instead of $X^{*}$, and $\epsilon$ is a measurement error. There is a rich literature on using density deconvolution for estimating the probability density function (pdf) $f_{X^{*}}$ (see, Meister, 2009, for a review). By contrast, the literature on estimation and inference for the cdf $F_{X^{*}}$ is relatively thin. Fan (1991) proposed a cdf estimator by integrating the deconvolution density estimator with some truncation. This truncation for the integral is circumvented in Hall and Lahiri (2008) (for the case where the pdf $f_{\epsilon}$ of $\epsilon$ is symmetric) and Dattner, Goldenshluger and Juditsky (2011) (for the case where $f_{\epsilon}$ is possibly asymmetric). Hall and Lahiri (2008) studied the $L_{2}$-risk properties of the cdf estimator. Dattner, Goldenshluger and Juditsky (2011) considered minimax rate optimal estimation of $F_{X^{*}}$. Both Hall and Lahiri (2008) and Dattner, Goldenshluger and Juditsky (2011) focused on the risk properties of the estimator $\tilde{F}_{X^{*}}\left(t_{0}\right)$ at a given $t_{0}$ and assumed known ordinary smooth densities for $f_{\epsilon}$. These papers demonstrate that, in contrast to the no measurement error case, the cdf estimator $\tilde{F}_{X^{*}}\left(t_{0}\right)$ typically converges to $F_{X^{*}}\left(t_{0}\right)$ at a nonparametric rate. On the other hand, Söhl and Trabs (2012) established a uniform central limit theorem for linear functionals of the

The authors would like to thank seminar participants at Aarhus, Durham, Queen Mary, Toulouse, UBC, Yale, and Econometric Society European Meeting at Lisbon for helpful comments. The authors also acknowledge helpful comments from an associate editor and anonymous referees. Financial support from the ERC Consolidator Grant (SNP 615882) is gratefully acknowledged (Otsu). 
deconvolution estimator that can be applied to derive a Donsker-type theorem, i.e., the weak convergence of $\sqrt{n}\left\{\tilde{F}_{X^{*}}(\cdot)-F_{X^{*}}(\cdot)\right\}$ to a Gaussian process. Söhl and Trabs (2012) considered the case of known ordinary smooth $f_{\epsilon}$, and for the Donsker-type result obtained therein, it is demanded that the Fourier transform $f_{\epsilon}^{\mathrm{ft}}$ satisfies $\left|f_{\epsilon}^{\mathrm{ft}}(\cdot)\right| \leq C|\cdot|^{-\beta}$ for some $\beta<1 / 2$ and $C>0$. The latter excludes the Laplace distribution, for instance. It must be emphasized that (except for Fan, 1991, on the truncated estimator) all these papers concentrate on the case of ordinary smooth and known $f_{\epsilon}$, so the cases of super smooth and/or unknown $f_{\epsilon}$ (with repeated measurements) are not covered.

In this paper, we investigate asymptotic and bootstrap approximations for the distributions of the maximal deviations $S_{n}=\sup _{t \in \mathcal{T}}\left|\tilde{F}_{X^{*}}(t)-F_{X^{*}}(t)\right|$ and $T_{n}=\sup _{t \in \mathcal{T}}\left|\hat{F}_{X^{*}}(t)-F_{X^{*}}(t)\right|$ in the sup-norm over some set $\mathcal{T}$, where $\tilde{F}_{X^{*}}$ is the deconvolution cdf estimator by Hall and Lahiri (2008) for known symmetric $f_{\epsilon}$ or Dattner, Goldenshluger and Juditsky (2011) for known possibly asymmetric $f_{\epsilon}$, and $\hat{F}_{X^{*}}$ is its adaptation to the case of unknown $f_{\epsilon}$ estimated by repeated measurements. Our analysis allows $f_{\epsilon}$ to be ordinary or super smooth, or to be unknown and estimated by repeated measurements. If $f_{\epsilon}$ is known to be symmetric, $\hat{F}_{X^{*}}$ is constructed by using Delaigle, Hall and Meister's (2008) estimator on the Fourier transform of $f_{\epsilon}$. If $f_{\epsilon}$ is possibly asymmetric, $\hat{F}_{X^{*}}$ is constructed by using Comte and Kappus' (2015) estimator, which is a regularized version of Li and Vuong's (1998) estimator based on the Kotlarski identity.

More specifically, our main contributions are described as follows: First, we propose a bootstrap method to approximate the distribution of $T_{n}$ and establish its asymptotic validity. This is not a trivial extension from the existing results. Compared to Hall and Lahiri (2008) and Dattner, Goldenshluger and Juditsky (2011), which studied pointwise estimation on $F_{X^{*}}$, we investigate uniform inference problems. Even for the same estimator, theoretical developments to analyze the pointwise and uniform properties of nonparametric estimators are very different (see, e.g., Giné and Nickl, 2016, for a general discussion on uniform inference). Furthermore, while these papers (and Söhl and Trabs (2012) mentioned above) focus on the case of known and ordinary smooth $f_{\epsilon}$, our analysis also covers the case of unknown and/or super smooth $f_{\epsilon}$. As is known in the literature, the analysis on the super smooth case is very different from the ordinary smooth case. Indeed, to the best of our knowledge, the limiting distributions of $S_{n}$ and $T_{n}$ for super smooth $f_{\epsilon}$ are still open questions. In this paper, we argue that by applying a novel idea in Chernozhukov, Chetverikov and Kato (2014) for constructing bootstrap-based confidence bands to our measurement error setup, we can establish the asymptotic validity of our bootstrap approximation for the distribution of $T_{n}$ without deriving its limiting distribution. Our bootstrap approximation allows $f_{\epsilon}$ to be possibly asymmetric at the cost of discarding some data to estimate $F_{X^{*}}$ (see, Section 3.1). 
Second, for the case of known $f_{\epsilon}$, we characterize the convergence rate of the bootstrap approximation error and find that it is of polynomial order under ordinary smooth errors, and logarithmic order under super smooth errors. Furthermore, for the case of known ordinary smooth $f_{\epsilon}$, we show that after suitable normalization, the asymptotic distribution of $S_{n}$ is characterized by the Gumbel distribution. Although the assumption of known $f_{\epsilon}$ is less realistic in empirical economic analyses, these results provide building blocks to establish our main results for the case of unknown $f_{\epsilon}$, and are also new in the literature of nonparametric deconvolution methods. In the context of density deconvolution, Bissantz, Dümbgen, Holzmann and Munk (2007) extended Bickel and Rosenblatt's (1973) construction of uniform confidence bands for densities to the classical measurement error model with the ordinary smooth $f_{\epsilon}$. A recent paper by Kato and Sasaki (2018) considered confidence bands of the pdf $f_{X^{*}}$ with unknown $f_{\epsilon}$. In contrast to these papers, we are concerned with inference on the cdf $F_{X^{*}} \cdot{ }^{1}$

Third, our approximation results on the distributions of $T_{n}$ and $S_{n}$ are applicable to various contexts, such as confidence bands for $F_{X^{*}}$ and its quantiles, and for performing various cdfbased tests such as goodness-of-fit tests for parametric models of $F_{X^{*}}$, two sample homogeneity tests, and tests for stochastic dominance. We emphasize that some inference problems, such as testing for stochastic dominance, are cumbersome to be handled by density-based methods. Also, even in cases where density-based methods are applicable (e.g., goodness-of-fit testing), the cdf-based methods are expected to have more desirable power properties. All these applications are new in the literature. For example, to the best of our knowledge, our stochastic dominance test is the first test in the literature to be robust to the presence of measurement errors. Dattner, Reiß and Trabs (2016) proposed a quantile estimator of $X^{*}$ and obtained its uniform convergence rate. This paper provides a confidence band for the quantile function of $X^{*}$.

This paper is organized as follows. In Section 2, we introduce our setup (Section 2.1) and present the main result, bootstrap approximation for the distribution of $T_{n}$ in the case of unknown symmetric $f_{\epsilon}$ (Section 2.2). In Section 3, we extend the results to the cases where $f_{\epsilon}$ is unknown and possibly asymmetric (Section 3.1) and direct observations from $f_{\epsilon}$ are available (Section 3.2). Section 4 presents additional theoretical results on the asymptotic properties of $S_{n}$ in the case of known $f_{\epsilon}$. Section 5 contains four applications of the main results: a confidence band for quantiles (Section 5.1), goodness-of-fit test for parametric models of $F_{X^{*}}$ (Section 5.2),

\footnotetext{
$\overline{{ }^{1} \text { In the context }}$ of nonparametric errors-in-variables regression analysis, Delaigle, Hall and Jamshidi (2015) proposed a bootstrap-based pointwise confidence interval of the regression function for the case of known $f_{\epsilon}$. A recent paper by Kato and Sasaki (2017) developed a bootstrap-based uniform confidence band for the case of unknown $f_{\epsilon}$. Also in the context of nonparametric inverse regression analysis, which also employs deconvolution methods, Birke, Bissantz and Holzmann (2010) and Proksh, Bissantz and Dette (2015) proposed confidence bands for the regression function. We refer Kato and Sasaki (2017) for an extensive survey on this literature. Finally, Kato, Sasaki and Ura (2019) proposed an alternative approach to construct a confidence band for $f_{X} *$ by utilizing linear moment restrictions obtained from Kotlarski's identity.
} 
homogeneity test for two samples (Section 5.3), and test for stochastic dominance (Section 5.4). Section 6 presents some simulation evidence. In Section 7, we consider a real data example. In particular, we employ the new test of stochastic dominance to study welfare changes of different population sub-groups using potentially mis-measured income data from Korea. All proofs are contained in the appendix.

\section{MAIN RESUlT}

2.1. Setup and estimator. We first introduce our basic setup. Suppose we observe a random sample $\left\{X_{i}\right\}_{i=1}^{n}$ generated from

$$
X=X^{*}+\epsilon
$$

where $X^{*}$ is an unobservable variable of interest and $\epsilon$ is its measurement error. Throughout the paper, $\epsilon$ is assumed to be independent of $X^{*}$ (i.e., $\epsilon$ is the classical measurement error). Let $\mathrm{i}=\sqrt{-1}$ and $f^{\mathrm{ft}}$ be the Fourier transform of a function $f$. If the pdf $f_{\epsilon}$ of $\epsilon$ is known, the pdf $f_{X^{*}}$ of $X^{*}$ can be estimated by the so-called deconvolution kernel density estimator (see, e.g., Stefanski and Carroll, 1990)

$$
\tilde{f}_{X^{*}}(t)=\frac{1}{n h} \sum_{i=1}^{n} \mathbb{K}\left(\frac{t-X_{i}}{h}\right), \quad \text { where } \mathbb{K}(u)=\frac{1}{2 \pi} \int_{-1}^{1} e^{-\mathrm{i} \omega u} \frac{K^{\mathrm{ft}}(\omega)}{f_{\epsilon}^{\mathrm{ft}}(\omega / h)} d \omega,
$$

where $h$ is a bandwidth and $K$ is a kernel function with $K^{\mathrm{ft}}$ supported on $[-1,1]$.

This section focuses on the case where $f_{\epsilon}$ is symmetric. The general case of possibly asymmetric $f_{\epsilon}$ will be discussed in Section 3.1. If $f_{\epsilon}$ is symmetric, integration of $\tilde{f}_{X^{*}}$ yields the following estimator for the $\operatorname{cdf} F_{X^{*}}$ of $X^{*}$ (see, Hall and Lahiri, 2008)

$$
\tilde{F}_{X^{*}}(t)=\frac{1}{2}+\frac{1}{n} \sum_{i=1}^{n} \mathbb{L}\left(\frac{t-X_{i}}{h}\right), \quad \text { where } \mathbb{L}(u)=\frac{1}{2 \pi} \int_{-1}^{1} \frac{\sin (\omega u)}{\omega} \frac{K^{\mathrm{ft}}(\omega)}{f_{\epsilon}^{\mathrm{ft}}(\omega / h)} d \omega .
$$

The estimator $\tilde{F}_{X^{*}}$ requires knowledge of the distribution of $\epsilon$, which is unrealistic in many applications. In general, however, $f_{\epsilon}$ cannot be identified by a single measurement. Identification of $f_{\epsilon}$ can be restored if we have two or more independent noisy measurements of the variable $X^{*}$. More specifically, suppose that we observe

$$
X_{i, j}=X_{i}^{*}+\epsilon_{i, j} \quad \text { for } j=1, \ldots, N_{i} \text { and } i=1, \ldots, n,
$$

where $X_{i}^{*}$ is the error-free variable and $\epsilon_{i, j}$ 's are independently distributed measurement errors from the density $f_{\epsilon}$. We thus have $N_{i}$ repeated measurements of each variable $X_{i}^{*}$. We shall assume that the number of repeated observations is bounded above (i.e., $N_{i} \leq C<\infty$ for all $i$ ). This assumption is not critical for our theory but allows us to simplify the proofs considerably. Since in practice the number of repeated measurements is small anyway, we do not pursue the 
generalization to growing $C$. Under the assumption that $f_{\epsilon}$ is symmetric, its Fourier transform $f_{\epsilon}^{\mathrm{ft}}$ can be estimated by (Delaigle, Hall and Meister, 2008)

$$
\hat{f}_{\epsilon}^{\mathrm{ft}}(\omega)=\left|\frac{1}{N} \sum_{i=1}^{n} \sum_{\left(j_{1}, j_{2}\right) \in \mathcal{J}_{i}}^{N_{i}} \cos \left\{\omega\left(X_{i, j_{1}}-X_{i, j_{2}}\right)\right\}\right|^{1 / 2}
$$

where $N=\frac{1}{2} \sum_{i=1}^{n} N_{i}\left(N_{i}-1\right), \mathcal{J}_{i}$ is the set of $\frac{1}{2} N_{i}\left(N_{i}-1\right)$ distinct pairs $\left(j_{1}, j_{2}\right)$ with $1 \leq j_{1}<$ $j_{2} \leq N$, and we ignore all the observations with $N_{i}=1$. By plugging this estimator into (2.3), we can estimate the cdf $F_{X^{*}}$ by

$$
\hat{F}_{X^{*}}(t)=\frac{1}{2}+\frac{1}{N} \sum_{i=1}^{n} \sum_{j=1}^{N_{i}} \hat{\mathbb{L}}\left(\frac{t-X_{i, j}}{h}\right), \quad \text { where } \hat{\mathbb{L}}(u)=\frac{1}{2 \pi} \int_{-1}^{1} \frac{\sin (\omega u)}{\omega} \frac{K^{\mathrm{ft}}(\omega)}{\hat{f}_{\epsilon}^{\mathrm{ft}}(\omega / h)} d \omega .
$$

In this section, we are concerned with approximation for the distribution of the maximal deviation

$$
T_{n}=\sup _{t \in \mathcal{T}}\left|\hat{F}_{X^{*}}(t)-F_{X^{*}}(t)\right|,
$$

under the sup-norm, where $\mathcal{T}$ is a compact interval specified by the researcher. A direct use of such approximation is construction of the confidence band for $F_{X^{*}}$ over $\mathcal{T}$. Several other ways to use this approximation are presented in Section 5.

2.2. Bootstrap approximation. In this section, we consider a bootstrap approximation for the distribution of $T_{n}$. To construct the bootstrap counterpart of $T_{n}$, we suggest resampling from the set of observed variables $\left\{X_{i, j}\right\}$ while keeping the estimated measurement error density $\hat{f}_{\epsilon}^{\mathrm{ft}}$ the same. More precisely, the bootstrap version of $\hat{F}_{X^{*}}$ is given by

$$
\hat{F}_{X^{*}}^{\#}(t)=\frac{1}{2}+\frac{1}{N} \sum_{i=1}^{n} \sum_{j=1}^{N_{i}} \hat{\mathbb{L}}\left(\frac{t-X_{i, j}^{\#}}{h}\right),
$$

where $X_{i, j}^{\#}$ is randomly drawn from the pooled observations $\left\{X_{i, j}\right\}$ with equal weights. Then the bootstrap counterpart of $T_{n}$ is obtained as $T_{n}^{\#}=\sup _{t \in \mathcal{T}}\left|\hat{F}_{X^{*}}^{\#}(t)-\hat{F}_{X^{*}}(t)\right|$.

To establish validity of the bootstrap approximation, we impose the following assumptions.

Assumption C. (i) $\left\{X_{i, j}\right\}_{i=1}^{n}$ is an i.i.d. sample from $X_{i, j}=X_{i}^{*}+\epsilon_{i, j}$ for $j=1, \ldots, N_{i}$, where $N_{i} \leq C<\infty$ for all $i$. $X_{i}^{*}$ and $\epsilon_{i, j}$ are independent for all $i$ and $j$. (ii) The densities $f_{X}, f_{X^{*}}$, and $f_{\epsilon}$ are bounded and continuous on $\mathbb{R}$, and $\inf _{t \in \mathcal{T}^{\delta}} f_{X}(t)>c$ for some $c>0$ and $\delta$-expansion $\mathcal{T}^{\delta}$ of $\mathcal{T}$. Also, $E\left|X^{*}\right|<\infty$ and $E|\epsilon|<\infty$. (iii) $\sup _{\omega \in \mathbb{R}}\left\{(1+|\omega|)^{\gamma}\left|f_{X^{*}}^{\mathrm{ft}}(\omega)\right|\right\}<C$ for some $\gamma, C>0$. (iv) $f_{\epsilon}^{\mathrm{ft}}(\omega) \neq 0$ for all $\omega \in \mathbb{R}, f_{\epsilon}^{\mathrm{ft}}(\omega)$ is differentiable at all $\omega \in \mathbb{R}$, and $f_{\epsilon}$ is an even function. (v) There exist $c \in(0,1)$ and $C>0$ such that $P\{|\epsilon| \geq M\} \leq C(\log M)^{-1 / c}$ for all $M>0$. 
Assumption C (i) is on the setup wherein we assume that $\epsilon$ is a classical measurement error. ${ }^{2}$ Assumption C (ii) is mild but excludes the Cauchy measurement error. This assumption is required for characterizing the bias of the estimator (see, e.g., Hall and Lahiri, 2008). The Cauchy measurement error is also ruled out in van Es and Uh (2005) who show pointwise asymptotic normality of the deconvolution density estimator. Assumption C (iii), analogous to the so-called Sobolev condition, is also used to characterize the rate for the bias term. Assumption C (iv) contains conditions on $f_{\epsilon}$. The first condition is common in the density deconvolution literature but may be relaxed by taking a ridge approach as in Hall and Meister (2007). The last condition, symmetry of $f_{\epsilon}$, is used not only to derive the cdf estimator in (2.3) as in Hall and Lahiri (2008) but also to obtain the estimator of $\hat{f}_{\epsilon}^{\mathrm{ft}}$ in (2.5). This condition will be relaxed in Section 3.1. Assumption $\mathrm{C}(\mathrm{v})$ is an additional regularity condition on the tail behavior of $\epsilon$. If $f_{\epsilon}$ is known, this assumption can be dropped.

We now present two classes of assumptions on the tail behavior of $f_{\epsilon}$. The first class of measurement error densities, called the ordinary smooth densities, are presented as follows.

Assumption OS. (i) There exist $\beta>1 / 2$ and $c, C, \omega_{0}>0$ such that

$$
c|\omega|^{-\beta} \leq\left|f_{\epsilon}^{\mathrm{ft}}(\omega)\right| \leq C|\omega|^{-\beta}
$$

for all $|\omega| \geq \omega_{0}$. (ii) $K$ is an even function with $K^{\mathrm{ft}}(\omega)=\left(1-\omega^{q}\right)^{r} \mathbb{I}\{|\omega| \leq 1\}$ for some $q, r \geq 2$. There exist $c_{1}, C_{1}>0$ such that

$$
n^{-1 / 4} h^{\beta-1 / 2} \int|\mathbb{K}(u)| d u<C_{1} n^{-c_{1}}
$$

for all $n$ large enough. Also, letting $\overline{\mathbb{K}}(u)=\frac{1}{\pi} \int_{0}^{1} \cos (\omega u) \frac{K^{\mathrm{ft}}(\omega)}{f_{\epsilon}^{\mathrm{ft}}(\omega / h)} \mathbb{I}\left\{|\omega| \geq h \omega_{0}\right\} d \omega$, it holds that

$$
h^{\beta-1 / 2} \int|\mathbb{K}(u)-\overline{\mathbb{K}}(u)| d u=O\left(h^{s}\right),
$$

for some $s>0$. (iii) As $n \rightarrow \infty$, it holds $h \rightarrow 0$, $\sqrt{n} h^{\beta-1 / 2} \rightarrow \infty, n^{\nu} h \rightarrow 0$ for some $\nu \in(0,1 / 2)$, and $n^{1+2 \xi} h^{2(\beta+\gamma)-1} \rightarrow 0$ for some $\xi>0$. (iv) As $n \rightarrow \infty$, it holds $\log n /\left(n h^{4 \beta}\right) \rightarrow 0$ and $n h^{4 \beta+1} \rightarrow \infty$. Also, $\gamma>\beta+1$.

Assumption OS (i) is a standard condition to characterize ordinary smooth densities. Note that we focus on the case of $\beta>1 / 2$, where the cdf estimator $\hat{F}_{X^{*}}$ converges at a nonparametric rate (Dattner, Goldenshluger and Juditsky, 2011). For example, the Laplace distribution corresponds to the case of $\beta=2$. Also it is known that if $\beta>1 / 2$, then $f_{\epsilon}$ is bounded and continuous. For the case of $\beta<1 / 2$, the estimator $\hat{F}_{X^{*}}$ typically converges at the $\sqrt{n}$-rate and a

\footnotetext{
${ }^{2}$ The independence assumption between $X_{i}^{*}$ and $\epsilon_{i, j}$ is standard but can be relaxed to the sub-independence assumption (see, Schennach, 2013). In the context of errors-in-variables regression analysis with repeated measurements, Schennach (2004) and $\mathrm{Hu}$ and Sasaki (2015) relaxed the independence assumption for the second and subsequent measurement errors $\epsilon_{i, j}$ with $j \geq 2$.
} 
Donsker-type theorem applies (Söhl and Trabs, 2012). Assumption OS (ii) contains conditions for the kernel function. The first condition specifies a particular form for $K$ that is commonly used in the literature (e.g., Delaigle and Hall, 2006). The second condition ensures that the deconvolution kernel $\mathbb{K}$ is $L_{1}$-integrable. The term $n^{-1 / 4}$ in (2.9) is required to ensure that the bootstrap counterpart $T_{n}^{\#}$ convergences to a Gaussian process at a polynomial rate in $n$ (see, Lemma 2 in the appendix). If $f_{\epsilon}^{\mathrm{ft}}$ is twice differentiable, applying the integration by parts formula twice gives

$$
\mathbb{K}(u)=\frac{1}{u^{2}} \int_{0}^{1} \cos (\omega u)\left\{\frac{K^{\mathrm{ft}}(\omega)}{f_{\epsilon}^{\mathrm{ft}}(\omega / h)}\right\}^{\prime \prime} d \omega,
$$

and a sufficient condition for $(2.9)$ is

$$
n^{-1 / 4} h^{\beta-1 / 2} \sup _{|\omega| \leq 1}\left|\left\{\frac{K^{\mathrm{ft}}(\omega)}{f_{\epsilon}^{\mathrm{ft}}(\omega / h)}\right\}^{\prime \prime}\right|=O\left(n^{-c_{1}}\right),
$$

for some $c_{1}>0$. The third condition in Assumption OS (ii) assures that $\mathbb{K}$ is well approximated by its trimmed version $\overline{\mathbb{K}}$. Since

$$
\int|\mathbb{K}(u)-\overline{\mathbb{K}}(u)| d u=\frac{1}{\pi} \int\left|\int_{0}^{h \omega_{0}} \cos (\omega u) \frac{K^{\mathrm{ft}}(\omega)}{f_{\epsilon}^{\mathrm{ft}}(\omega / h)} d \omega\right| d u,
$$

applying the integration by parts formula twice again implies that a sufficient condition for (2.10) is given by

$$
h^{\beta+1 / 2} \sup _{|\omega| \leq h \omega_{0}} \max \left\{\left|\left(\frac{K^{\mathrm{ft}}(\omega)}{f_{\epsilon}^{\mathrm{ft}}(\omega / h)}\right)^{\prime}\right|,\left|\left(\frac{K^{\mathrm{ft}}(\omega)}{f_{\epsilon}^{\mathrm{ft}}(\omega / h)}\right)^{\prime \prime}\right|\right\}=O\left(h^{s}\right),
$$

for some $s>0$. Based on the above sufficient conditions, it is possible to show that Assumption OS (ii) is satisfied by a large class of ordinary smooth error distributions including Laplace and its convolutions. Intuitively, these conditions mean that $f_{\epsilon}^{\mathrm{ft}}$ should not oscillate too wildly around its trend implied by the ordinary smooth density.

Assumption OS (iii) contains conditions for the bandwidth $h$. The first condition, $h \rightarrow 0$, is a standard requirement for controlling the smoothing bias. The second condition, $\sqrt{n} h^{\beta-1 / 2} \rightarrow \infty$, is imposed to ensure the variance goes to zero. The third condition, $n^{\nu} h \rightarrow 0$, is a technical condition on the bandwidth to ensure that it is not too large; this is easily satisfied for the undersmoothing bandwidth choice. Finally the last condition, $n^{1+2 \xi} h^{2(\beta+\gamma)-1} \rightarrow 0$, ensures that the bandwidth is undersmoothed relative to the MSE optimal choice, which is $h \propto n^{1 / 2(\beta+\gamma)-1}$ (see, Hall and Lahiri, 2008). For example, if $h \propto n^{-a}$, then these conditions are satisfied with $a \in\left(\max \left\{\frac{1+2 \xi}{2 \beta-1+2 \gamma}, \nu\right\}, \frac{1}{2 \beta-1}\right)$, which typically holds true by choosing $\xi$ and $\nu$ small enough.

Finally, Assumption OS (iv) lists additional conditions to deal with the case of unknown $f_{\epsilon}$. The requirement $\gamma>\beta+1$ says that $f_{X^{*}}$ is smoother than $f_{\epsilon}$ by up to a derivative. As 
shown in Lemma 2 in the appendix, this ensures that the error from estimating $f_{\epsilon}^{\mathrm{ft}}$ is asymptotically negligible. Also, we note that the conditions $n h^{4 \beta+1} \rightarrow \infty$ (Assumption C (iv)) and $n^{1+2 \xi} h^{2(\beta+\gamma)-1} \rightarrow 0$ for some $\xi>0$ (Assumption OS (iii)) hold simultaneously only if $\gamma>\beta+1$. Delaigle, Hall and Meister (2008) imposed a similar condition to obtain the MSE optimal estimator of $f_{X^{*}}$ under repeated measurements. They also showed that when the converse holds (i.e, $\gamma<\beta+1$ ), the estimation error of $f_{\epsilon}^{\mathrm{ft}}$ dominates that of $f_{X}^{*}$, and the estimator $\hat{f}_{\epsilon}^{\mathrm{ft}}$ for $f_{\epsilon}^{\mathrm{ft}}$ given in (2.5) is no longer optimal (see, p. 673 of Delaigle, Hall and Meister, 2008). The same comment applies when $f_{X}^{*}$ is ordinary smooth but $f_{\epsilon}$ is super smooth.

The second class of measurement error densities, called the super smooth densities, are presented as follows.

Assumption SS. (i) There exist $\mu, c, C, \omega_{0}, \lambda>0$ and $\lambda_{0} \in \mathbb{R}$ such that

$$
c|\omega|^{\lambda_{0}} \exp \left(-|\omega|^{\lambda} / \mu\right) \leq\left|f_{\epsilon}^{\mathrm{ft}}(\omega)\right| \leq C|\omega|^{\lambda_{0}} \exp \left(-|\omega|^{\lambda} / \mu\right)
$$

for all $|\omega| \geq \omega_{0}$. (ii) $K$ is an even function with $K^{\mathrm{ft}}(\omega)=\left(1-\omega^{q}\right)^{r} \mathbb{I}\{|\omega| \leq 1\}$ for some $q, r \geq 2$. There exist $\mu_{1}>2 \mu$ and $c_{1}, C_{1}<\infty$ such that

$$
\frac{1}{\varsigma(h)} \int|\mathbb{K}(u)| d u<C_{1} h^{-c_{1}} \exp \left(\frac{1}{\mu_{1} h^{\lambda}}\right)
$$

for all $n$ large enough, where

$$
\varsigma(h)=h^{\vartheta} \exp \left(\frac{1}{\mu h^{\lambda}}\right)
$$

with $\vartheta=\lambda(r+1 / 2)+\lambda_{0}+1 / 2$. Also, letting $\overline{\mathbb{K}}(u)=\frac{1}{\pi} \int_{0}^{1} \cos (\omega u) \frac{K^{\mathrm{ft}}(\omega)}{f_{\epsilon}^{\mathrm{ft}}(\omega / h)} \mathbb{I}\left\{|\omega| \geq h \omega_{0}\right\} d \omega$, it holds that

$$
\frac{1}{\varsigma(h)} \int|\mathbb{K}(u)-\overline{\mathbb{K}}(u)| d u=O\left(n^{-s}\right),
$$

for some $s>0$. (iii) $h=\left(\frac{\mu}{2} \log n+\mu \vartheta_{1} \log \log n\right)^{-1 / \lambda}$ for some $\vartheta_{1} \in((\vartheta-\gamma) / \lambda+1, \vartheta / \lambda)$. (iv) $\vartheta_{1}<2 \lambda_{0} / \lambda$ and $\int\left|\omega^{a} f_{X^{*}}^{\mathrm{ft}}(\omega) / f_{\epsilon}^{\mathrm{ft}}(\omega)\right|^{2} d \omega<\infty$ for some $a>\vartheta-\lambda_{0}+1$.

Assumption SS (i) is a standard condition to characterize super smooth densities. Assumption SS (ii) contains conditions on the kernel function, and similar comments apply as for the ordinary smooth case. The condition $\mu_{1}>2 \mu$ is required to guarantee that the bootstrap counterpart $T_{n}^{\#}$ convergences to a Gaussian process at a polynomial rate in $n$ (see Lemma 9 in the appendix). If $f_{\epsilon}^{\mathrm{ft}}$ is twice differentiable, a sufficient condition for $(2.11)$ is

$$
\frac{1}{\varsigma(h)} \sup _{|\omega| \leq 1}\left|\left(\frac{K^{\mathrm{ft}}(\omega)}{f_{\epsilon}^{\mathrm{ft}}(\omega / h)}\right)^{\prime \prime}\right|=O\left(h^{-a} \exp \left(\frac{1}{\mu_{1} h^{\lambda}}\right)\right),
$$


for some $a>0$. Also, a sufficient condition for (2.13) is

$$
\exp \left(-\frac{1}{\mu h^{\lambda}}\right) \sup _{|\omega| \leq h \omega_{0}} \max \left\{\left|\left(\frac{K^{\mathrm{ft}}(\omega)}{f_{\epsilon}^{\mathrm{ft}}(\omega / h)}\right)^{\prime}\right|,\left|\left(\frac{K^{\mathrm{ft}}(\omega)}{f_{\epsilon}^{\mathrm{ft}}(\omega / h)}\right)^{\prime \prime}\right|\right\}=O\left(n^{-a_{1}}\right),
$$

for some $a_{1}>0$. For instance, these conditions are satisfied if

$$
\sup _{|\omega| \leq 1} \max \left\{\left|A^{\prime}(\omega / h)\right|,\left|A^{\prime \prime}(\omega / h)\right|\right\}=O\left(h^{-a_{1}} \exp \left(\frac{1}{\mu_{1} h^{\lambda}}\right)\right),
$$

for some $a_{1}>0$, where $A(\omega)=\frac{\exp \left(-|\omega|^{\lambda} / \mu\right)}{f_{\epsilon}^{\mathrm{ft}}(\omega)}$. Based on (2.14), we can see that Assumption SS (ii) is satisfied by a large class of super smooth error distributions including Gaussian and its convolutions. Since the function $A$ inherits the differentiability properties of $f_{\epsilon}^{\mathrm{ft}}$, the condition (2.14) intuitively means that $f_{\epsilon}^{\mathrm{ft}}$ should not oscillate too wildly around its trend implied by the super smooth density.

Assumption SS (iii) is on the bandwidth $h$. Compared to the ordinary smooth case, we need stricter conditions on the bandwidth to ensure the bias decays to 0 faster than the variance. Note that the MSE optimal bandwidth choice in this instance is $h \propto(\log n)^{-1 / \lambda}$; the additional constants and the $(\log \log n)$ term are needed to ensure undersmoothing. We also note that the condition on $\vartheta_{1}$ implicitly demands that $\gamma>\lambda$ to ensure that the interval for $\vartheta_{1}$ is non-empty. The lower bound for $\vartheta_{1}$ is derived from the condition for undersmoothing (see discussion above Lemma 11 in the appendix), which demands that the bias decay to 0 at a rate faster than $\log n$, i.e., $\frac{\sqrt{n} h^{\gamma}}{\varsigma(h)}=O(\log n)^{-c}$ for some $c>1$. Indeed, by the definition of $\varsigma(h)$ and the expression of $h$ in Assumption SS (iii), we have $\frac{\sqrt{n} h^{\gamma}}{\varsigma(h)}(\log n)^{c} \sim\left(\frac{\mu}{2}\right)^{\frac{\gamma-\vartheta}{\lambda}}(\log n)^{\frac{\gamma-\vartheta}{\lambda}+\vartheta_{1}+c}$, which implies the lower bound on $\vartheta_{1}$. The upper bound on $\vartheta_{1}$ is obtained by the requirement that $\sqrt{n} / \varsigma(h) \rightarrow 0$ so as to ensure the variance decays to 0 .

Finally, Assumption SS (iv) lists additional conditions to deal with the case of unknown $f_{\epsilon}$.

Let $\hat{c}_{\alpha}$ denote the $(1-\alpha)$-th quantile of the bootstrap statistic $T_{n}^{\#}$. Under the above assumptions, asymptotic validity of the bootstrap approximation is established as follows. ${ }^{3}$

Theorem 1. Suppose that Assumptions $C$ and either $O S$ or SS hold. Then

$$
P\left\{T_{n} \leq \hat{c}_{\alpha}\right\} \geq 1-\alpha-o(1)
$$

Remark 1. Based on this theorem, we can construct an asymptotic confidence band for $F_{X^{*}}$ over $\mathcal{T}$ with level $\alpha$ as $\left[\hat{F}_{X^{*}}(t) \pm \hat{c}_{\alpha}\right]$ for $t \in \mathcal{T}$. To implement the bootstrap approximation in this theorem, we need to choose the bandwidth $h$. For estimation of the $\operatorname{cdf} F_{X^{*}}\left(t_{0}\right)$ at a

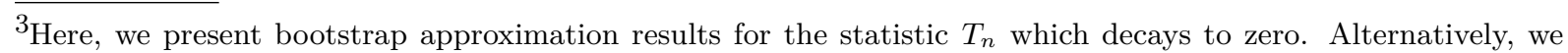
could have normalized $T_{n}$ without affecting any of the conclusions. This is analogous to whether we present the bootstrap approximation for the non-normalized object $\hat{\theta}-\theta$ or the normalized one $\sqrt{n}(\hat{\theta}-\theta)$, where $\theta$ is some parameter and $\hat{\theta}$ its estimator.
} 
given $t_{0}$, Hall and Lahiri (2008) suggested choosing $h$ to minimize the approximate integrated MSE based on the normal reference distribution on $X^{*}$. For estimation of the quantile function of $X^{*}$, Dattner, Reiß and Trabs (2016) developed an adaptive method to choose $h$ based on Lepski's (1990) method. In Section 6 for simulations, we suggest a bandwidth selection rule based on Bissantz, Dümbgen, Holzmann and Munk (2007). The basic idea is to estimate the ideal bandwidth that minimizes the maximal deviation between $\hat{F}_{X^{*}}$ and $F_{X^{*}}$ under the supnorm by utilizing a series of estimates $\hat{F}_{X^{*}}$ based on different values of $h$.

Remark 2. Theorem 1 says that the approximation error on the right hand side of (2.15) is of order $o(1)$. On the other hand, Theorem 3 below for the case of known $f_{\epsilon}$ provides more accurate orders. The main obstacle for obtaining an accurate order of the approximation error in the present setup is that the contrast $\hat{F}_{X^{*}}(t)-\tilde{F}_{X^{*}}(t)$ arising from the estimation of $f_{\epsilon}$ is currently bounded by a stochastic term (see Lemma 2) rather than a deterministic one.

\section{Extensions}

3.1. Possibly asymmetric measurement error distribution. In this section, we extend our bootstrap inference method in Section 2 to the case where the measurement error density $f_{\epsilon}$ is unknown and possibly asymmetric around zero. In particular, by using repeated measurements, we estimate $f_{\epsilon}^{\mathrm{ft}}$ by a nonparametric deconvolution estimator in Comte and Kappus (2015), which is a modified version of Li and Vuong's (1998) estimator based on Kotlarski's identify. Then we argue that an adjusted version of our bootstrap approach using subsamples can be applied to conduct valid inference. Although this method works under more general situations than the one in the last section, we need to discard some data to estimate $F_{X^{*}}$ to achieve the asymptotic validity of our bootstrap method.

Let us introduce some notation. For possibly asymmetric $f_{\epsilon}$, integration of $\tilde{f}_{X *}$ in $(2.2)$ yields the following estimator for $F_{X^{*}}$ (Dattner, Goldenshluger and Juditsky, 2011)

$$
\tilde{F}_{X^{*}}^{A}(t)=\frac{1}{2}-\frac{1}{n} \sum_{i=1}^{n} \mathbb{L}^{A}\left(\frac{t-X_{i}}{h}\right), \quad \text { where } \mathbb{L}^{A}(u)=\frac{1}{\pi} \int_{0}^{1} \frac{1}{\omega} \operatorname{Im}\left[\frac{e^{-\mathrm{i} \omega u}}{f_{\epsilon}^{\mathrm{ft}}(\omega / h)}\right] K^{\mathrm{ft}}(\omega) d \omega
$$

where $\operatorname{Im}[\cdot]$ stands for the imaginary part. If $f_{\epsilon}$ is symmetric, this definition coincides with the one in (2.3). We consider a feasible version of $\tilde{F}_{X^{*}}^{A}$ by estimating $f_{\epsilon}^{\mathrm{ft}}$ in (3.1).

To simplify the presentation, we concentrate on the case where two repeated measurements on $X^{*}$ are available, i.e., set $N_{i}=2$ in (2.1). Let $\hat{\psi}\left(\omega_{1}, \omega_{2}\right)=\frac{1}{n} \sum_{i=1}^{n} e^{\mathrm{i} \omega_{1} X_{i, 1}+\mathrm{i} \omega_{2} X_{i, 2}}$ be the empirical characteristic function of the observables. Li and Vuong (1998) proposed to estimate $f_{\epsilon}^{\mathrm{ft}}$ by

$$
\hat{f}_{\epsilon, L V}^{\mathrm{ft}}(\omega)=\frac{\hat{\psi}(\omega, 0)}{\hat{\varphi}_{L V}(\omega)}, \quad \text { where } \quad \hat{\varphi}_{L V}(\omega)=\exp \int_{0}^{\omega} \frac{\partial \hat{\psi}\left(0, \omega_{2}\right) / \partial \omega_{1}}{\hat{\psi}\left(0, \omega_{2}\right)} d \omega_{2}
$$


Although the uniform convergence rate of $\hat{f}_{\epsilon, L V}^{\mathrm{ft}}$ is known in the literature (Li and Vuong, 1998, for the bounded support case, and Kurisu and Otsu, 2019, for a general case), our theoretical development for the validity of bootstrap inference also requires certain $L_{2}$ convergence results (as in Lemmas 4 and 5 in the appendix). The $L_{2}$ convergence rate of Li and Vuong's (1998) estimator is an open problem, and here we consider a modified version of $\mathrm{Li}$ and Vuong (1998) proposed by Comte and Kappus (2015) (say, $\check{f}_{\epsilon}^{\mathrm{ft}}$ ). The basic idea of Comte and Kappus (2015) is to regularize $\hat{\varphi}_{L V}(\omega)$ as

$$
\hat{\varphi}_{K P}(\omega)=\exp \int_{0}^{\omega} \frac{\partial \hat{\psi}\left(0, \omega_{2}\right) / \partial \omega_{1}}{\check{\psi}\left(0, \omega_{2}\right)} d \omega_{2}, \quad \text { with } \quad \check{\psi}\left(0, \omega_{2}\right)=\frac{\hat{\psi}\left(0, \omega_{2}\right)}{\min \left\{1, \sqrt{n}\left|\hat{\psi}\left(0, \omega_{2}\right)\right|\right\}},
$$

where the term, $\min \left\{1, \sqrt{n}\left|\hat{\psi}\left(0, \omega_{2}\right)\right|\right\}$, circumvents unfavorable effects caused by small values of the denominator of $\hat{f}_{\epsilon, L V}^{\mathrm{ft}}$. Based on this, Comte and Kappus' (2015) estimator $f_{\epsilon}^{\mathrm{ft}}$ is defined as

$$
\check{f}_{\epsilon}^{\mathrm{ft}}(\omega)=\frac{\hat{\psi}(\omega, 0)}{\check{\varphi}(\omega)}
$$

where $\check{\varphi}(\omega)=\frac{\check{\varphi}_{1}(\omega)}{\min \left\{1, \sqrt{n}\left|\check{\varphi}_{1}(\omega)\right|\right\}}$ and $\check{\varphi}_{1}(\omega)=\frac{\hat{\varphi}_{K P}(\omega)}{\max \left\{1,\left|\hat{\varphi}_{K P}(\omega)\right|\right\}}$. The additional regularization term, $\max \left\{1,\left|\hat{\varphi}_{K P}(\omega)\right|\right\}$, is introduced to improve the quality of the estimator by imposing the fact that the estimand is a characteristic function, which should not take values larger than one. Comte and Kappus (2015) and Kurisu and Otsu (2019) derived the $L_{2}$ and uniform convergence rates of $\check{f}_{\epsilon}^{\mathrm{ft}}$, respectively, under mild conditions allowing unbounded support for $X^{*}$ and $\epsilon$.

We first consider the estimator of the cdf $F_{X^{*}}$ by plugging $\check{f}_{\epsilon}^{\mathrm{ft}}$ into $\tilde{F}_{X^{*}}^{A}$ in (3.1):

$$
\check{F}_{X^{*}}(t)=\frac{1}{2}-\frac{1}{n} \sum_{i=1}^{n} \frac{1}{2} \sum_{j=1}^{2} \check{\mathbb{L}}\left(\frac{t-X_{i, j}}{h}\right), \quad \text { where } \check{\mathbb{L}}(u)=\frac{1}{\pi} \int_{0}^{1} \frac{1}{\omega} \operatorname{Im}\left[\frac{e^{-\mathrm{i} \omega u}}{\check{f}_{\epsilon}^{\mathrm{ft}}(\omega / h)}\right] K^{\mathrm{ft}}(\omega) d \omega .
$$

To extend our bootstrap approach in Section 2, we utilize the uniform convergence results of $\check{f}_{\epsilon}^{\mathrm{ft}}$ in Kurisu and Otsu (2019), and impose the following conditions.

Assumption C'. (i) $f_{\epsilon}^{\mathrm{ft}}(\omega) \neq 0$ for all $\omega \in \mathbb{R}, E[\epsilon]=0$, and $E\left[\left|X_{1,1}\right|^{2+\zeta}\right]<\infty$ for some $\zeta>0$.

Assumption OS'. (i) There exist $\gamma>1 / 2$ and $c_{x}, C_{x}, \omega_{x}>0$ such that

$$
c_{x}|\omega|^{-\gamma} \leq\left|f_{X^{*}}^{\mathrm{ft}}(\omega)\right| \leq C_{x}|\omega|^{-\gamma},
$$

for all $|\omega| \geq \omega_{x}$.

Assumption SS'. (i) There exist $\mu_{x}, c_{x}, C_{x}, \omega_{x}, \lambda_{x}>0$ and $\lambda_{0 x} \in \mathbb{R}$ such that

$$
c_{x}|\omega|^{\lambda_{0 x}} \exp \left(-|\omega|^{\lambda_{x}} / \mu_{x}\right) \leq\left|f_{X^{*}}^{\mathrm{ft}}(\omega)\right| \leq C_{x}|\omega|^{\lambda_{0 x}} \exp \left(-|\omega|^{\lambda_{x}} / \mu_{x}\right),
$$

for all $|\omega| \geq \omega_{x}$. 
The assumption $E[\epsilon]=0$ is employed to identify $f_{\epsilon}^{\mathrm{ft}}$ by Kotlarski's identity (Li and Vuong, 1998), and $E\left[\left|X_{1,1}\right|^{2+\zeta}\right]<\infty$ is an additional condition to apply the results in Kurisu and Otsu (2019). Since the estimator $\check{f}_{\epsilon}^{\mathrm{ft}}$ is defined by the ratios of the (regularized) empirical averages, we introduce the lower and upper bounds of the characteristic function $f_{X^{*}}^{\mathrm{ft}}$, as in Assumptions OS' (i) and SS' (i), to control the estimation error in $\check{f}_{\epsilon}^{\mathrm{ft}}$. By applying Kurisu and Otsu (2019), the uniform convergence rates of $\check{f}_{\epsilon}^{\mathrm{ft}}$ are obtained as follows.

Lemma 1. [Adaptation of Kurisu and Otsu (2019)]

(i): Under Assumptions C(i)-(ii), $C^{\prime}(i)$, OS (i), and $O S^{\prime}(i)$, it holds

$$
\sup _{\omega \in\left[-h^{-1}, h^{-1}\right]}\left|\check{f}_{\epsilon}^{\mathrm{ft}}(\omega)-f_{\epsilon}^{\mathrm{ft}}(\omega)\right|=O_{p}\left(n^{-1 / 2} h^{-3 \gamma-2 \beta-1} \log h^{-1}\right) .
$$

(ii): Under Assumptions C (i)-(ii), $C^{\prime}(i), S S$ (i), and $S S^{\prime}(i)$, it holds

$$
\sup _{\omega \in\left[-h^{-1}, h^{-1}\right]}\left|\check{f}_{\epsilon}^{\mathrm{ft}}(\omega)-f_{\epsilon}^{\mathrm{ft}}(\omega)\right|=O_{p}\left(n^{-1 / 2} h^{-1+3 \lambda_{0 x}+2 \lambda_{0}}\left(\log h^{-1}\right) \exp \left(\frac{3 h^{-\lambda_{x}}}{\mu_{x}}+\frac{2 h^{-\lambda}}{\mu}\right)\right) .
$$

Note that this lemma does not require $f_{\epsilon}$ to be symmetric. The above rates are typically faster than the ones in Li and Vuong (1998) or Bonhomme and Robin (2010). However, they are still too slow to adapt the bootstrap approach in Section 2. For example, for the ordinary smooth case, the main term $\sup _{t \in \mathcal{T}}\left|\tilde{F}_{X^{*}}^{A}(t)-F_{X^{*}}(t)\right|$ is of order $O_{p}\left(n^{-1 / 2} h^{-\beta+1 / 2}\right)$ (see Lemma 6 in the appendix), which is faster than the uniform rate in Lemma 1 (i). Thus, the bootstrap approach proposed in the last section cannot be directly applied to the object $\sup _{t \in \mathcal{T}}\left|\check{F}_{X^{*}}(t)-F_{X^{*}}(t)\right|$.

To deal with this issue, we consider the following modified version of $\check{F}_{X^{*}}$ :

$$
\check{F}_{X^{*}}^{A}(t)=\frac{1}{2}-\frac{1}{m} \sum_{i=1}^{m} \frac{1}{2} \sum_{j=1}^{2} \check{\mathbb{L}}\left(\frac{t-X_{i, j}}{h}\right),
$$

where $\left\{X_{i, 1}, X_{i, 2}\right\}_{i=1}^{m}$ is a subsample of size $m<n$ from the original one. Note that $\check{f}_{\epsilon}^{\mathrm{ft}}$ appearing in the construction of $\check{\mathbb{L}}(\cdot)$ is computed based on the full sample of size $n$. By suitably choosing $m$, the main term $\sup _{t \in \mathcal{T}}\left|\tilde{F}_{X^{*}}^{A}(t)-F_{X^{*}}(t)\right|$ using the subsample will dominate the estimation error of $\check{f}_{\epsilon}^{\mathrm{ft}}$ using the full sample.

In this subsection, we use the following assumptions. For any (complex-valued) function on $\mathbb{R}$, let $\|f\|_{p}=\left(\int_{\mathbb{R}}|f(x)|^{p} d x\right)^{1 / p}$ for $p>0$.

Assumption C'. (ii) $f_{\epsilon}^{\mathrm{ft}}(\omega)$ is differentiable at all $\omega \in \mathbb{R}$. (iii) There exists a positive integer $p>2$ such that

$$
\left(\left\|f_{X^{*}}^{\mathrm{ft}^{\prime \prime}} f_{\epsilon}^{\mathrm{ft}}\right\|_{1}+\left\|E\left[\epsilon^{2}\right] f_{X^{*}}^{\mathrm{ft}} f_{\epsilon}^{\mathrm{ft}}\right\|_{1}+\left\|f_{X^{*}}^{\mathrm{ft}} f_{\epsilon}^{\mathrm{ft}}\right\|_{2}^{2}\right)^{p}+E\left[\left|X_{1,1}\right|^{4 p}\right] \leq C_{1},
$$


for some $C_{1}>0$, where $f_{X^{*}}^{\mathrm{ft}^{\prime}}$ and $f_{X^{*}}^{\mathrm{ft}^{\prime \prime}}$ are the first and second derivatives of $f_{X^{*}}^{\mathrm{ft}}$. (iv) The characteristic function $\psi\left(\omega_{1}, \omega_{2}\right)=E\left[e^{\mathrm{i}\left(\omega_{1} X_{1,1}+\omega_{2} X_{1,2}\right)}\right]$ satisfies $\left\|\partial \log \psi(0, \cdot) / \partial \omega_{1}\right\|_{2} \leq C_{2}$ for some $C_{2}>0$.

The (square) integrability conditions on $f_{X^{*}}^{\mathrm{ft}}, f_{\epsilon}^{\mathrm{ft}}$ and $\partial \log \psi(0, \cdot) / \partial \omega_{1}$ are mild. Additional conditions, also assumed in Comte and Kappus (2015), are imposed to control the weighted $L_{2}$ risk for $\check{f}_{\epsilon}^{\mathrm{ft}}$ (see Lemma 4 in the appendix).

Assumption OS'. (ii) Assumption OS (ii) holds true after replacing $n$ with $m$ in (2.9), (iii) As $m \rightarrow \infty$, it holds $h \rightarrow 0, \sqrt{m} h^{\beta-1 / 2} \rightarrow \infty, m^{\nu} h \rightarrow 0$ for some $\nu \in(0,1 / 2)$, and $m^{1+2 \xi} h^{2(\beta+\gamma)-1} \rightarrow 0$ for some $\xi>0$, (iv) There exists a positive integer $p>2$ such that $n^{p-2} h^{(2 \beta+2 \gamma+1)(p-1)+2 \gamma} \rightarrow \infty$ as $n \rightarrow \infty$. (v) $n^{-1 / 2} h^{-3 \gamma-3 \beta-1} \log h^{-1} \rightarrow 0$ as $n \rightarrow \infty$.

Assumption SS'. (ii) Assumption SS (ii) holds true after replacing $n$ with $m$ in (2.13). Also, Assumption SS (iii)-(iv) hold true. (iii) There exists a positive integer $p>2$ such that

$$
n^{p-2} h^{-2 \lambda_{0 x}-(p-1)\left(2 \lambda_{0 x}+2 \lambda_{0}-[(1-\lambda) \vee 0]\right)} \exp \left(\frac{(-4(p-1)+2) h^{-\lambda}}{\mu_{x} \wedge \mu}\right) \rightarrow \infty \text { as } n \rightarrow \infty .
$$

(iv) $n^{-1 / 2} h^{-1+3 \lambda_{0 x}+3 \lambda_{0}}\left(\log h^{-1}\right) \exp \left(\frac{3 h^{-\lambda_{x}}}{\mu_{x}}+\frac{3 h^{-\lambda}}{\mu}\right) \rightarrow 0$ as $n \rightarrow \infty$.

These assumptions are modifications of Assumptions OS and SS for the subsample-based statistic $\check{F}_{X^{*}}^{A}$. For example, if $m=n^{b}$ with $0<b<1$ and $h=n^{-a}$ with $a>0$, Assumption OS' (iii) is satisfied when $0<a<\frac{1}{2(3 \gamma+3 \beta+1)}$ and $0<b<\frac{2 \gamma+2 \beta-1}{2(3 \gamma+3 \beta+1)}$. We use Assumptions OS' (iv) and SS' (iii) to simplify the results of Lemmas 4 and 5 in the appendix, which provide the wighted $L^{2}$ risk of $\check{f}_{\epsilon}^{\mathrm{ft}}$. Assumptions OS' (v) and SS' (iv) are imposed to show the uniform consistency of $\left|f_{\epsilon}^{\mathrm{ft}}(\omega) / \check{f}_{\epsilon}^{\mathrm{ft}}(\omega)\right|$ on $\left[-h^{-1}, h^{-1}\right]$.

Let $T_{n}^{A}=\sup _{t \in \mathcal{T}}\left|\check{F}_{X^{*}}^{A}(t)-F_{X^{*}}(t)\right|$, and $\hat{c}_{\alpha}^{A}$ denote the $(1-\alpha)$-th quantile of the bootstrap statistic $T_{n}^{A \#}=\sup _{t \in \mathcal{T}}\left|\check{F}_{X^{*}}^{A \#}(t)-\check{F}_{X^{*}}^{A}(t)\right|$, where

$$
\check{F}_{X^{*}}^{A \#}(t)=\frac{1}{2}-\frac{1}{m} \sum_{i=1}^{m} \frac{1}{2} \sum_{j=1}^{2} \check{\mathbb{L}}\left(\frac{t-X_{i, j}^{\#}}{h}\right) .
$$

The validity of our bootstrap procedure is presented as follows.

Theorem 2. Suppose

(i): Assumptions $C$ (i)-(ii) C', OS (i), and $O S^{\prime}$ hold true, and $m n^{\zeta_{0}-1} h^{2 \beta-2 \gamma-1} \rightarrow 0$ as $n \rightarrow \infty$ for some $0<\zeta_{0}<1$, or

(ii): Assumptions $C$ (i)-(ii) $C^{\prime}, S S($ i $)$, and $S S^{\prime}$ hold true, and $m n^{\zeta_{1}-1}(\log n)^{2 r+1}(\log \log n)^{2} \rightarrow$ 0 as $n \rightarrow \infty$ for some $0<\zeta_{1}<1$.

Then

$$
P\left\{T_{n}^{A} \leq \hat{c}_{\alpha}^{A}\right\} \geq 1-\alpha-o(1)
$$


The same comments to Theorem 2 apply. For example, we can construct an asymptotic confidence band for $F_{X^{*}}$ over $\mathcal{T}$ with level $\alpha$ as $\left[\check{F}_{X^{*}}^{A}(t) \pm \hat{c}_{\alpha}^{A}\right]$ for $t \in \mathcal{T}$.

3.2. Case of directly observable measurement errors. In some applications, a separate independent experiment may give us direct observations from $f_{\epsilon}$ (see, e.g., Efromovich, 1997, and Neumann, 1997). If we have $\ell$ independent observations $\left(\epsilon_{1}, \ldots, \epsilon_{\ell}\right)$ from $f_{\epsilon}$, the Fourier transform $f_{\epsilon}^{\mathrm{ft}}$ can be estimated as

$$
\bar{f}_{\epsilon}^{\mathrm{ft}}(t)=\frac{1}{\ell} \sum_{i=1}^{\ell} \exp \left(\mathrm{i} t \epsilon_{i}\right) .
$$

We may use this estimator to obtain an estimator for $F_{X^{*}}$ by replacing $f_{\epsilon}^{\mathrm{ft}}$ in $(2.3)$ with $\bar{f}_{\epsilon}^{\mathrm{ft}}$. A bootstrap counterpart can be constructed as in (2.8) by replacing $\hat{f}_{\epsilon}^{\mathrm{ft}}$ with $\bar{f}_{\epsilon}^{\mathrm{ft}}$. The asymptotic validity of such bootstrap approximation can be established by replacing Assumption OS (iv) with the following one.

Assumption OS. (iv)' As $n \rightarrow \infty$, it holds $\ell \rightarrow \infty, \sqrt{(n / \ell)} h^{\beta-1 / 2} \log (1 / h) \rightarrow 0$ and $\log n /\left(\ell h^{4 \beta}\right) \rightarrow$ 0 .

For the case of super smooth $f_{\epsilon}$, we can supplement Assumption SS with the following condition on $\ell$.

Assumption SS. (v) As $n \rightarrow \infty$, it holds $\ell / n \rightarrow$ a for some $a \in(0,1)$.

The formal proof for validity of the resulting bootstrap follows by analogous arguments as in the proof of Theorem 1, and is therefore omitted.

\section{Case of Known measurement error Distribution}

In this section, we present additional theoretical results on the asymptotic properties of the cdf estimator $\tilde{F}_{X^{*}}$ in (2.3) for the case of known measurement error distribution. The results in this section are building blocks for our main result in Theorem 1. Also, the results here are new in the statistics literature on nonparametric deconvolution methods, and are of interest in their own right.

This section is concerned with approximation for the distribution of the maximal deviation

$$
S_{n}=\sup _{t \in \mathcal{T}}\left|\tilde{F}_{X^{*}}(t)-F_{X^{*}}(t)\right|,
$$

under the sup-norm, where $\mathcal{T}$ is a compact interval specified by the researcher.

We first establish asymptotic validity of a bootstrap approximation for $S_{n}$. Consider a nonparametric bootstrap resample $\left\{X_{i}^{\#}\right\}_{i=1}^{n}$ from $\left\{X_{i}\right\}_{i=1}^{n}$ with equal weights. The bootstrap counterpart of $S_{n}$ is given by $S_{n}^{\#}=\sup _{t \in \mathcal{T}}\left|\tilde{F}_{X^{*}}^{\#}(t)-\tilde{F}_{X^{*}}(t)\right|$, where $\tilde{F}_{X^{*}}^{\#}$ is defined as in (2.3) using 
$X_{i}^{\#}$. Let $\tilde{c}_{\alpha}$ denote the $(1-\alpha)$-th quantile of the bootstrap statistic $T_{n}^{\#}$. The validity of the bootstrap approximation is established as follows.

Theorem 3. Suppose that Assumption C (i)-(iv) holds true. Then

$$
P\left\{S_{n} \leq \tilde{c}_{\alpha}\right\} \geq 1-\alpha-\delta_{n}
$$

for some positive sequence $\delta_{n}=O\left(n^{-c}\right)$ (under Assumption $O S(i)$-(iii)) or $\delta_{n}=O\left((\log n)^{-c}\right)$ (under Assumption SS (i)-(iii)) with $c>0$.

Remark 3. Based on this theorem, we can construct an asymptotic confidence band for $F_{X^{*}}$ over $\mathcal{T}$ with level $\alpha$ as $\mathcal{C}_{n}(t)=\left[\tilde{F}_{X^{*}}(t) \pm \tilde{c}_{\alpha}\right]$ for $t \in \mathcal{T}$, which satisfies

$$
P\left\{F_{X^{*}}(t) \in \mathcal{C}_{n}(t) \text { for all } t \in \mathcal{T}\right\} \geq 1-\alpha-\delta_{n},
$$

for $\delta_{n}=O\left(n^{-c}\right)$ (under Assumption OS (i)-(iii)) or $\delta_{n}=O\left((\log n)^{-c}\right)$ (under Assumption SS (i)-(iii)) with some $c>0$. The approximation error $\delta_{n}$ provides bounds on the extent of the discrepancy in size (from a nominal level of $\alpha$ ) when using the bootstrap critical values. We note that this is distinct from the convergence rate of the cdf estimator $\tilde{F}_{X^{*}}$. We can see that $\delta_{n}$ is of polynomial order under Assumption OS (the ordinary smooth case), and of logarithmic order under Assumption SS (the super smooth case). We also note from the proof of the theorem that the slower approximation rate of $\delta_{n}$ for the super-smooth case is solely due to the estimation bias for $F_{X^{*}}(t)$; if bias correction were possible, the bootstrap approximation error would be of polynomial order in both cases. This is so even as the variance of the cdf estimator $\tilde{F}_{X^{*}}$ itself goes to 0 at a logarithmic rate.

4.1. Asymptotic Gumbel approximation for ordinary smooth case. For the ordinary smooth case, it is also possible to characterize the asymptotic distribution of the standardized object

$$
s_{n}=\sqrt{n h^{2 \beta-1}} \sup _{t \in \mathcal{T}}\left|f_{X}(t)^{-1 / 2}\left\{\tilde{F}_{X^{*}}(t)-F_{X^{*}}(t)\right\}\right|,
$$

using the Gumbel distribution. Note that to obtain $s_{n}$, we normalize the discrepancy $\tilde{F}_{X^{*}}(t)-$ $F_{X^{*}}(t)$ by $f_{X}(t)^{-1 / 2}$, the density function of the mismeasured data $X .{ }^{4}$ Such normalization by $f_{X}(t)^{-1 / 2}$ also appears in the case of the deconvolution density estimator for $f_{X^{*}}$ over $t \in \mathcal{T}$ (Bissantz, Dümbgen, Holzmann and Munk, 2007). Under additional assumptions, listed in Assumption G in Appendix C, we can follow similar steps as in Bickel and Rosenblatt (1973) and Bissantz, Dümbgen, Holzmann and Munk (2007) to show the following result.

\footnotetext{
${ }^{4}$ The reason for this is that the empirical distribution, which is used to sum up the observations to compute $\tilde{F}_{X^{*}}$, is based on the cdf $F_{X}$ of the observed data $X$.
} 
Theorem 4. Suppose that Assumptions C (i)-(iv), OS (i)-(iii), and G hold, and $(n h)^{-1}(\log n)^{3} \rightarrow$ 0 as $n \rightarrow \infty$. Then

$$
P\left\{(-2 \log h)^{1 / 2}\left(B^{-1 / 2} s_{n}-b_{n}\right) \leq c\right\} \rightarrow \exp (-2 \exp (-c))
$$

for all $c \in \mathbb{R}$, where the constant $B$ and sequence $b_{n}$ are defined in Appendix $C$ (eq. (C.2)).

See Appendix C for a detailed discussion of Assumption G and for the proof of this theorem.

Remark 4. As shown in (4.4), the limiting behavior of $s_{n}$ is characterized by the Gumbel distribution. Also based on this theorem, we can see that the convergence rate of $\sup _{t \in \mathcal{T}} \mid \tilde{F}_{X^{*}}(t)-$ $F_{X^{*}}(t) \mid$ is of order $n^{-1 / 2} h^{-\beta+1 / 2} \sqrt{\log h^{-1}}$. Note that in analogous setups, the convergence rate of $\sup _{t \in \mathcal{T}}\left|\tilde{f}_{X^{*}}(t)-f_{X^{*}}(t)\right|$ for the deconvolution pdf estimator in (2.2) is of order $n^{-1 / 2} h^{-\beta-1 / 2} \sqrt{\log h^{-1}}$ (see, Theorem 1 in Bissantz, Dümbgen, Holzmann and Munk, 2007, and Corollary 1 in Kato and Sasaki, 2018). Thus, the uniform convergence rate of the cdf estimator is faster than that of the pdf estimator by the factor of $h$. See Dattner, Goldenshluger and Juditsky (2011) for analogous results on the minimax rate for pointwise estimation problems for a given $t$.

Remark 5. Based on (4.4), and the conventional kernel density estimator $\hat{f}_{X}$ for $f_{X}$, we can also obtain an asymptotically valid critical value to conduct inference. For example, the asymptotic confidence band at level $\alpha$ for $F_{X^{*}}$ is given by

$$
\mathcal{C}_{n}^{G}(t)=\left[\tilde{F}_{X^{*}}(t) \pm \sqrt{\frac{B \hat{f}_{X}(t)}{n h^{2 \beta-1}}}\left\{c_{\alpha}^{G}(-2 \log h)^{-1 / 2}+b_{n}\right\}\right],
$$

for $t \in \mathcal{T}$, where $c_{\alpha}^{G}$ solves $\exp \left(-2 \exp \left(-c_{\alpha}^{G}\right)\right)=\alpha$. However, as discussed in the next remark, the asymptotic Gumbel approximation requires additional assumptions and tends to be less accurate than the bootstrap approximation.

Remark 6. Compared to the bootstrap approximation, the asymptotic Gumbel approximation has two drawbacks. First, the Gumbel approximation requires an additional assumption (Assumption G). Second, as indicated by Bissantz, Dümbgen, Holzmann and Munk (2007), the approximation error (i.e., $\delta_{n}$ in (4.2) for the bootstrap approximation) given by (4.4) is typically a logarithmic rate even under Assumption OS, and therefore tends to be less accurate than the bootstrap approximation in (4.2). This contrast between the asymptotic Gumbel and bootstrap approximations was first clarified by Chernozhukov, Chetverikov and Kato (2014) for construction of confidence bands on the density with no measurement error. Kato and Sasaki (2018) extended their results for confidence bands on the pdf $f_{X^{*}}$ with unknown $f_{\epsilon}$. We obtain analogous results for confidence bands on the $\operatorname{cdf} F_{X^{*}}$. We also note that in contrast to 
Chernozhukov, Chetverikov and Kato (2014) and Kato and Sasaki (2018) who employed Gaussian multiplier bootstrap methods, Theorem 3 shows validity of the conventional nonparametric bootstrap. Accordingly, the techniques used in the proof of Theorem 3 are quite different: in particular, we employ Komlós, Major and Tusnády's (1975) coupling along with anti-concentration inequalities for Gaussian processes (Chernozhukov, Chetverikov and Kato, 2015) while the latter employ the Slepian-Stein type coupling for suprema of empirical processes constructed in Chernozhukov, Chetverikov and Kato (2014). Finally, we also obtain deterministic bounds on the approximation error of the bootstrap; to the best of our knowledge this is new in the literature on deconvolution.

Remark 7. We note that the asymptotic Gumbel approximation in (4.4) is available only for the ordinary smooth case. It remains an open question whether we can establish such an asymptotic approximation for the super smooth case. As discussed in Bissantz, Dümbgen, Holzmann and Munk (2007, p. 486) for density deconvolution, the main difficulty is that the limiting form of the deconvolution kernel (eq. (C.1) in Appendix C) is not available for the super smooth case. On the other hand, as shown in Theorem 3, we emphasize that the bootstrap approximation is valid even for the super smooth case.

\section{Applications}

5.1. Confidence band for quantile function. In addition to the confidence band for $F_{X^{*}}$, the results in the previous sections can be utilized to obtain the confidence band for the quantile function of $X^{*}$. Hall and Lahiri (2008) proposed estimating the $u$-th quantile $Q(u)=F_{X^{*}}^{-1}(u)$ by

$$
\tilde{Q}(u)=\sup \left\{t: \tilde{F}_{X^{*}}^{\mathrm{m}}(t) \leq u\right\},
$$

where $\tilde{F}_{X^{*}}^{m}(t)=\sup _{y \leq t} \tilde{F}_{X^{*}}(y)$ is a monotone version of $\tilde{F}_{X^{*}}(t)$. To obtain the confidence band for the quantile function $Q(u)$ over some interval $\left[u_{1}, u_{2}\right]$, we impose the following assumptions.

Assumption Q. (i) $F_{X^{*}}^{-1}(u)$ exists and is unique for all $u \in\left[u_{1}, u_{2}\right]$ such that $0<u_{1}<u_{2}<1$. There exists an interval $\mathcal{H}$ satisfying $F_{X^{*}}^{-1}\left[u_{1}-\varepsilon, u_{2}+\varepsilon\right] \subset \mathcal{H}$ for some $\varepsilon>0, \inf _{x \in \mathcal{H}} f_{X}(x)>0$, and $0<\inf _{x \in \mathcal{H}} f_{X^{*}}(x) \leq \sup _{x \in \mathcal{H}} f_{X^{*}}(x)<\infty$. (ii) $\sup _{x \in \mathcal{H}}\left|f_{X^{*}}(x+\delta)-f_{X^{*}}(x)\right| \leq M|\delta|^{a}$ for all $\delta$ sufficiently small, with $a>0$ (under Assumption OS) and $a=1$ (under Assumption SS).

Based on these assumptions, we can obtain the asymptotic confidence bands for the quantile function as follows.

Theorem 5. Suppose that Assumptions C, Q, and either OS or SS hold true. Then,

$$
P\left\{\tilde{Q}(u)-\frac{\tilde{c}_{\alpha}}{\tilde{f}_{X^{*}}(\tilde{Q}(u))} \leq Q(u) \leq \tilde{Q}(u)+\frac{\tilde{c}_{\alpha}}{\tilde{f}_{X^{*}}(\tilde{Q}(u))} \text { for all } u \in\left[u_{1}, u_{2}\right]\right\} \geq 1-\alpha-o(1) .
$$


Remark 8. For the case of known $f_{\epsilon}$, Dattner, Reiß and Trabs (2016) obtained the uniform convergence rate of their quantile estimator, say $\bar{Q}(u)$, based on the M-estimation method. In particular, Dattner, Reiß and Trabs (2016, Proposition 2.6) obtained that under an MSE optimal choice of the bandwidth,

$$
\sup _{u \in\left[u_{1}, u_{2}\right]}|\bar{Q}(u)-Q(u)|=O_{p}\left(\left(\frac{\log n}{n}\right)^{\frac{\gamma}{2(\beta+\gamma)-1}}\right) .
$$

Thus, Theorem 5 is complementary in that it provides a confidence band for $Q(u)$ over $u \in$ $\left[u_{1}, u_{2}\right]$. Note that as with the case of the cdf, we require under-smoothing to obtain the asymptotically valid confidence band, which excludes the MSE optimal bandwidth.

5.2. Goodness-of-fit testing. Another useful application of our results is goodness-of-fit testing on parametric models for $F_{X^{*}}$. Consider a parametric model $\left\{G_{X^{*}}(\cdot, \theta): \theta \in \Theta\right\}$ for the distribution of the error-free variable $X^{*}$ of interest. For simplicity, suppose the measurement error density $f_{\epsilon}$ is known as in Section 4. Our method can be adapted to the case of unknown $f_{\epsilon}$. The goodness-of-fit testing problem of our interest is

$$
H_{0}: F_{X^{*}}(t)=G_{X^{*}}(t, \theta) \text { over } t \in \mathcal{T} \text { for some } \theta \in \Theta
$$

against negation of $H_{0}$. Let $\hat{\theta}$ be some $\sqrt{n}$-consistent estimator of the true parameter $\theta_{0}$ under $H_{0}$. A typical example of $\hat{\theta}$ is the maximum likelihood estimator using the density function $\int g_{X^{*}}(t-a, \theta) f_{\epsilon}(a) d a$ on the observable $X$, where $g_{X^{*}}$ is the density of $G_{X^{*}}$.

To test $H_{0}$, we can employ the Kolmogorov-type statistic

$$
K_{n}=\sup _{t \in \mathcal{T}}\left|\tilde{F}_{X^{*}}(t)-G_{X^{*}}(t, \hat{\theta})\right|,
$$

and its bootstrap counterpart is given by

$$
K_{n}^{\#}=\sup _{t \in \mathcal{T}}\left|\tilde{F}_{X^{*}}^{\#}(t)-G_{X^{*}}\left(t, \hat{\theta}^{\#}\right)\right|,
$$

where $\tilde{F}_{X^{*}}^{\#}$ and $\hat{\theta}^{\#}$ are computed by the (parametric) bootstrap resample $\left\{X_{i}^{\#}\right\}_{i=1}^{n}$ from $X^{\#}=$ $X_{*}^{\#}+\epsilon^{\#}$ with $X_{*}^{\#} \sim G_{X^{*}}(\cdot, \hat{\theta})$ and $\epsilon^{\#} \sim f_{\epsilon}$. In contrast to the no measurement error case, the cdf estimator $\tilde{F}_{X^{*}}$ converges at a slower rate than $\sqrt{n}$. Therefore, if $\hat{\theta}$ is $\sqrt{n}$-consistent, then the estimation error of $\hat{\theta}$ is negligible under $H_{0}$, and the validity of the bootstrap critical value follows by a modification of the proof of Theorem 3 . The result is summarized in the following corollary. Let $\tilde{c}_{\alpha}^{K}$ be the $(1-\alpha)$-th quantile of $K_{n}^{\#}$.

Corollary 1. Suppose that Assumption C (i)-(iv) holds true, the null $H_{0}$ is satisfied at $\theta_{0}$, $\sqrt{n}\left(\hat{\theta}-\theta_{0}\right)=O_{p}(1)$, and the density of $G_{X^{*}}(\cdot, \theta)$ is bounded for all $\theta$ in a neighborhood of $\theta_{0}$. 
Then

$$
P\left\{K_{n}>\tilde{c}_{\alpha}^{K}\right\} \leq \alpha+\delta_{n},
$$

for some positive sequence $\delta_{n}=O\left(n^{-c}\right)$ (under Assumption $O S(i)$-(iii)) or $\delta_{n}=O\left((\log n)^{-c}\right)$ (under Assumption SS (i)-(iii)) with $c>0$.

Consistency of the test can be shown analogously. If $f_{\epsilon}$ is unknown but repeated measurements on $X^{*}$ are available, an analogous result holds true by replacing $\tilde{F}_{X^{*}}$ and $\tilde{F}_{X^{*}}^{\#}$ with $\hat{F}_{X^{*}}$ and $\hat{F}_{X^{*}}^{\#}$, respectively.

5.3. Homogeneity test. Our bootstrap and asymptotic approximation results can be extended to two sample problems. Let $\left\{X_{i}\right\}_{i=1}^{n}$ and $\left\{Y_{i}\right\}_{i=1}^{m}$ be two independent samples of $X$ and $Y$. $X$ is generated as in (2.1). Also $Y$ is generated as

$$
Y=Y^{*}+\delta
$$

where $Y^{*}$ is the unobservable error-free variable with the distribution function $F_{Y^{*}}$ and $\delta$ is its measurement error. We assume $\delta$ is independent of $Y^{*}$. Suppose we wish to test the homogeneity hypothesis

$$
H_{0}: F_{X^{*}}(t)=F_{Y^{*}}(t) \quad \text { for all } t \in \mathcal{T},
$$

against the negation of $H_{0}$. The Kolmogorov-type statistic presented in the last subsection can be modified as follows

$$
S_{n, m}=\sup _{t \in \mathcal{T}}\left|\tilde{F}_{X^{*}}(t)-\tilde{F}_{Y^{*}}(t)\right|,
$$

where $\tilde{F}_{Y^{*}}$ is the estimator for $F_{Y^{*}}$ as in $(2.3)$ using the sample $\left\{Y_{i}\right\}_{i=1}^{m}$. In this case, the bootstrap counterpart of $S_{n, m}$ is given by

$$
S_{n, m}^{\#}=\sup _{t \in \mathcal{T}}\left|\tilde{F}_{X^{*}}^{\#}(t)-\tilde{F}_{Y^{*}}^{\#}(t)-\left\{\tilde{F}_{X^{*}}(t)-\tilde{F}_{Y^{*}}(t)\right\}\right|,
$$

where $\tilde{F}_{Y^{*}}^{\#}$, obtained for the sample $\left\{Y_{i}\right\}_{i=1}^{m}$, is defined in the same manner as $\tilde{F}_{X^{*}}^{\#}$. The $(1-\alpha)$-th quantile $\tilde{c}_{\alpha}^{S}$ of $S_{n, m}^{\#}$ provides an asymptotically valid critical value as follows.

Corollary 2. Suppose that Assumption $C(i)$-(iv) holds true for both $X=X^{*}+\epsilon$ and $Y=Y^{*}+\delta$, and that $n /(n+m) \rightarrow \tau \in(0,1)$ as $n, m \rightarrow \infty$. Then under $H_{0}$,

$$
P\left\{S_{n, m}>\tilde{c}_{\alpha}^{S}\right\} \leq \alpha+\delta_{n, m}
$$

for some positive sequence $\delta_{n, m}=O\left(n^{-c}\right)$ (under Assumption OS (i)-(iii) for both $\epsilon$ and $\delta$ ) or $\delta_{n, m}=O\left((\log n)^{-c}\right)$ (under Assumption SS (i)-(iii) for both $\epsilon$ and $\left.\delta\right)$ with $c>0$.

An analogous result is available for the case of unknown $f_{\epsilon}$ by replacing $\tilde{F}_{X^{*}}$ and $\tilde{F}_{Y^{*}}$ with their estimated versions. Also, if we wish to test the homogeneity hypothesis $H_{0}$ but $Y$ has no 
measurement error (i.e., $Y=Y^{*}$ ), we can replace $\tilde{F}_{Y^{*}}$ with the empirical distribution function of the sample $\left\{Y_{i}\right\}_{i=1}^{m}$.

5.4. Stochastic dominance test. Another intriguing application of our main results is testing the hypothesis of the (first-order) stochastic dominance

$$
H_{0}: F_{X^{*}}(t) \leq F_{Y^{*}}(t) \text { for all } t \in \mathcal{T}
$$

against the negation of $H_{0}$. By modifying the Kolmogorov-type test in Section 5.3, the test statistic for (5.1) and its bootstrap counterpart are given by

$$
\begin{aligned}
D_{n, m} & =\sup _{t \in \mathcal{T}}\left\{\tilde{F}_{X^{*}}(t)-\tilde{F}_{Y^{*}}(t)\right\}, \\
D_{n, m}^{\#} & =\sup _{t \in \mathcal{T}}\left\{\tilde{F}_{X}^{\#}(t)-\tilde{F}_{Y}^{\#}(t)-\left\{\tilde{F}_{X}(t)-\tilde{F}_{Y}(t)\right\}\right\},
\end{aligned}
$$

where $\tilde{F}_{X}^{\#}$ and $\tilde{F}_{Y}^{\#}$ are computed as in $(2.3)$ using nonparametric bootstrap resamples $\left\{X_{i}^{\#}\right\}_{i=1}^{n}$ and $\left\{Y_{i}^{\#}\right\}_{i=1}^{m}$ from $\left\{X_{i}\right\}_{i=1}^{n}$ and $\left\{Y_{i}\right\}_{i=1}^{m}$, respectively.

Let $\tilde{c}_{\alpha}^{D}$ denote the $(1-\alpha)$-th quantile of the bootstrap statistic $D_{n, m}^{\#}$. The bootstrap validity of our stochastic dominance test is established as follows.

Theorem 6. Suppose that Assumption $C$ (i)-(iv) holds true for both $X=X^{*}+\epsilon$ and $Y=Y^{*}+\delta$, and that $n /(n+m) \rightarrow \tau \in(0,1)$ as $n, m \rightarrow \infty$.

(i): Under $H_{0}$,

$$
P\left\{D_{n, m}>\tilde{c}_{\alpha}^{D}\right\} \leq \alpha+\varrho_{n, m},
$$

for some positive sequence $\varrho_{n, m}=O\left(n^{-c}\right)$ (under Assumption OS (i)-(iii) for both $\epsilon$ and $\delta$ ) or $\varrho_{n, m}=O\left((\log n)^{-c}\right)$ (under Assumption $S S$ (i)-(iii) for both $\epsilon$ and $\delta$ ) with $c>0$.

(ii): Let $\mathcal{P}_{0}$ be the set of probability measures of $(X, Y)$ satisfying $H_{0}$ (but $f_{\delta}$ and $f_{\epsilon}$ are fixed) and

$$
\begin{aligned}
& 0<c_{X} \leq \inf _{t \in \mathcal{T}} f_{X}(t) \leq \sup _{t \in \mathcal{T}} f_{X}(t) \leq C_{X}<\infty \\
& 0<c_{Y} \leq \inf _{t \in \mathcal{T}} f_{Y}(t) \leq \sup _{t \in \mathcal{T}} f_{Y}(t) \leq C_{Y}<\infty \\
& \sup _{\omega \in \mathbb{R}}\left\{(1+|\omega|)^{\gamma_{X}}\left|f_{X^{*}}^{\mathrm{ft}}(\omega)\right|\right\} \leq M_{X}<\infty \\
& \sup _{\omega \in \mathbb{R}}\left\{(1+|\omega|)^{\gamma_{Y}}\left|f_{Y^{*}}^{\mathrm{ft}}(\omega)\right|\right\} \leq M_{Y}<\infty
\end{aligned}
$$

for some $c_{X}, c_{Y}, \gamma_{X}, \gamma_{Y}, C_{X}, C_{Y}, M_{X}, M_{Y}>0$ that are independent of $\left(f_{X}, f_{Y}\right)$. Then

$$
\sup _{P \in \mathcal{P}_{0}} P\left\{D_{n, m}>\tilde{c}_{\alpha}^{D}\right\} \leq \alpha+\varrho_{n, m}
$$


for some positive sequence $\varrho_{n, m}=O\left(n^{-c}\right)$ (under Assumption OS (i)-(iii)) or $\varrho_{n, m}=$ $O\left((\log n)^{-c}\right)$ (under Assumption SS (i)-(iii)) with $c>0$.

(iii): Under the alternative $H_{1}$ (i.e., $H_{0}$ is false) and either Assumption OS (i)-(iii) or SS (i)-(iii),

$$
P\left\{D_{n, m}>\tilde{c}_{\alpha}^{D}\right\} \rightarrow 1
$$

Remark 9. Based on the proof of Theorem 6 (iii), we can characterize some local power properties. Suppose that both measurement errors are ordinary smooth. For any sequence $M_{n} \rightarrow \infty, D_{n, m}$ is consistent (i.e., $P\left\{D_{n, m}>\tilde{c}_{\alpha}^{D}\right\} \rightarrow 1$ ) against local alternatives of the form

$$
H_{1 n}: F_{Y^{*}}(t)>F_{X^{*}}(t)+M_{n} \gamma_{n} \text { for some } t \in \mathcal{T},
$$

where

$$
\gamma_{n}=n^{-1 / 2} \max \left\{h_{X}^{1 / 2-\beta_{X}} \sqrt{\log \left(1 / h_{X}\right)}, h_{Y}^{1 / 2-\beta_{Y}} \sqrt{\log \left(1 / h_{Y}\right)}\right\},
$$

and $h_{X}$ and $h_{Y}$ are (possibly different) bandwidths for the estimators $\tilde{F}_{X^{*}}$ and $\tilde{F}_{Y^{*}}$, respectively. A similar expression is available for $\gamma_{n}$ in the super smooth case with $h_{X}^{\beta_{X}-1 / 2}$ and $h_{Y}^{\beta_{Y}-1 / 2}$ replaced by $\varsigma_{X}^{-1}\left(h_{X}\right)$ and $\varsigma_{Y}^{-1}\left(h_{Y}\right)$ respectively. Finally in the mixed error case, i.e when one of the errors is ordinary smooth while the other is super-smooth, the value of $\gamma_{n}$ is determined by the super-smooth error (e.g., $\gamma_{n}=n^{-1 / 2} \varsigma_{X}\left(h_{X}\right) \sqrt{\log \left(1 / h_{X}\right)}$ if $\epsilon$ is super-smooth).

\section{Simulation}

In this section, we investigate the finite sample performance of the bootstrap uniform confidence band discussed in Section 3 using simulation experiments.

6.1. Simulation designs. We generate data from the model (2.1), where the unobserved variable of interest $X^{*}$ is drawn from the normal distribution $N\left(0, \sigma_{X^{*}}^{2}\right)$ and the measurement error $\varepsilon$ is drawn from the Laplace distribution $L\left(0, \sigma_{\varepsilon}^{2}\right)$ or the normal distribution $N\left(0, \sigma_{\varepsilon}^{2}\right)$. We fix $\sigma_{X^{*}}=1$ and choose $\sigma_{\varepsilon}$ so that the 'signal-to-noise ratio (SNR)' is given by $\sigma_{X^{*}} / \sigma_{\varepsilon}=1,2,3 .^{5}$ We use the kernel function $K$ defined by

$$
K(\omega)=\frac{48 \cos \omega}{\pi \omega^{4}}\left(1-\frac{15}{\omega^{2}}\right)-\frac{144 \sin \omega}{\pi \omega^{5}}\left(2-\frac{5}{\omega^{2}}\right),
$$

whose Fourier transformation is given by $K^{\mathrm{ft}}(\omega)=\left(1-\omega^{2}\right)^{3} \cdot \mathbb{I}\{|\omega| \leq 1\}$. We consider four different sample sizes $n=100,250,500,1000$ and three different confidence levels $1-\alpha=$ $0.80,0.90,0.95$. The number of simulation and bootstrap repetitions are 2000 and 1000, respectively. We compute the coverage probabilities of our confidence bands for $F_{X^{*}}$ over the interval $\left[-2 \sigma_{X^{*}}, 2 \sigma_{X^{*}}\right]$.

\footnotetext{
${ }^{5}$ The signal-to-noise ratio can be also defined as the ratio $\sigma_{X} / \sigma_{\varepsilon}=\sigma_{X^{*}} / \sigma_{\varepsilon}+1$. Our definition of SNR follows from Bissantz, Dümbgen, Holzmann and Munk (2007); see also Kato and Sasaki (2018).
} 
6.2. Bandwidth choice. We adapt the bandwidth selection method of Bissantz, Dümbgen, Holzmann and Munk (2007, Section 5.2) to the cdf estimation. It aims at estimating the bandwidth $h_{\text {opt }}$, which minimizes the $L_{\infty}$-distance between $\tilde{F}_{X^{*}}$ and the underlying true cdf $F_{X^{*}}$. First we consider $J$ different bandwidths: $h_{j}=h_{0} j / J$ for $j=1,2, \ldots, J$, where $h_{0}$ is a pilot bandwidth. A pilot bandwidth is an over-smoothing bandwidth obtained by multiplying $\gamma>1$ to the normal reference rule of Hall and Lahiri (2008, Section 4.2). The normal reference rule was originally suggested by Delaigle and Gijbels (2004) to estimate density functions, and was modified by Hall and Lahiri (2008) to the setting of estimating distribution functions. For $j=2, \ldots, J$, define the distances

$$
L_{\infty}\left(\tilde{F}_{X^{*}}, F_{X^{*}}\right)=\left\|\tilde{F}_{X^{*}}-F_{X^{*}}\right\|_{\infty}, \quad d_{j-1, j}^{(\infty)}=\left\|\tilde{F}_{X^{*}, j-1}-\tilde{F}_{X^{*}, j}\right\|_{\infty},
$$

where $\tilde{F}_{X^{*}, j}$ denotes the deconvolution estimator (2.3) with bandwidth $h=h_{j}$ and $\|\cdot\|_{\infty}$ denotes the supremum norm. For over-smoothing bandwidths $h>h_{\mathrm{opt}}, L_{\infty}\left(\tilde{F}_{X^{*}}, F_{X^{*}}\right)$ changes only moderately with increasing bandwidth, while with undersmoothing bandwidths $h<h_{\text {opt }}$ the distance suddenly increases with decreasing bandwidth. Based on this observation, Bissantz, Dümbgen, Holzmann and Munk (2007, Section 5.2) suggest to estimate $h \approx h_{\mathrm{opt}}$ by choosing the largest bandwidth at which $d_{j-1, j}^{(\infty)}$ is more than $\tau(\tau>1)$ times greater than $d_{J-1, J}^{(\infty)}$. In our simulations, we choose $J=20$ (number of bandwidths), $\tau=3$, and $\gamma=1.5$. (We find that the simulation results are insensitive to the precise choice of the tuning parameters $J, \tau$, and $\gamma$.)

Figures 1 and 2 illustrate the distances over different bandwidths for 3 different random samples with the measurement error drawn from the Laplace and normal distributions, respectively. A comparison of two plots in the figures indicate that the bandwidth at which $d_{j-1, j}^{(\infty)}$ changes suddenly (marked by a circle, square, or star) is a good indicator of the bandwidth at which the true distance $L_{\infty}$ is about to stagnate. A further investigation of the theoretical properties of the bandwidth selection rule would be interesting, but is beyond the scope of our paper; see also Bissantz, Dümbgen, Holzmann and Munk (2007, p.497).

6.3. Simulation results. Table 1 presents the empirical coverage probabilities of our bootstrap confidence bands. As a benchmark, it also reports the coverage probabilities of the bootstrap confidence bands using the true $X^{*}$. The simulated probabilities are generally close to the nominal confidence levels. As we expected, the coverage errors tend to be smaller when the sample size is larger or when the SNR is larger.

Figures 3 and 4 depict some typical examples for the true cdf $\left(\mathrm{CDF}, F_{X^{*}}\right)$, deconvolution cdf estimate (ECDF, $\tilde{F}_{X^{*}}$ ), and uniform confidence bands $(\mathrm{CB})$, when the latent true distribution is standard normal and the measurement errors are drawn from Laplace and normal distributions. They show that the uniform confidence bands perform reasonably well even for small sample 

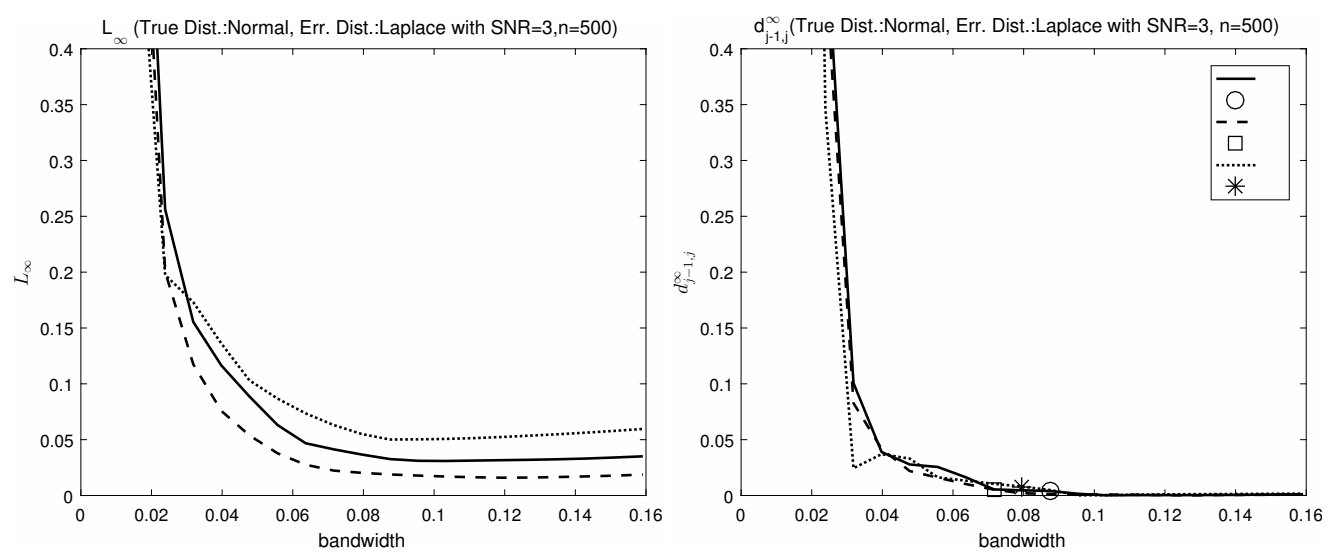

Figure 6.1. $L_{\infty}$ and $d_{j-1, j}^{\infty}$ distances under Laplace error
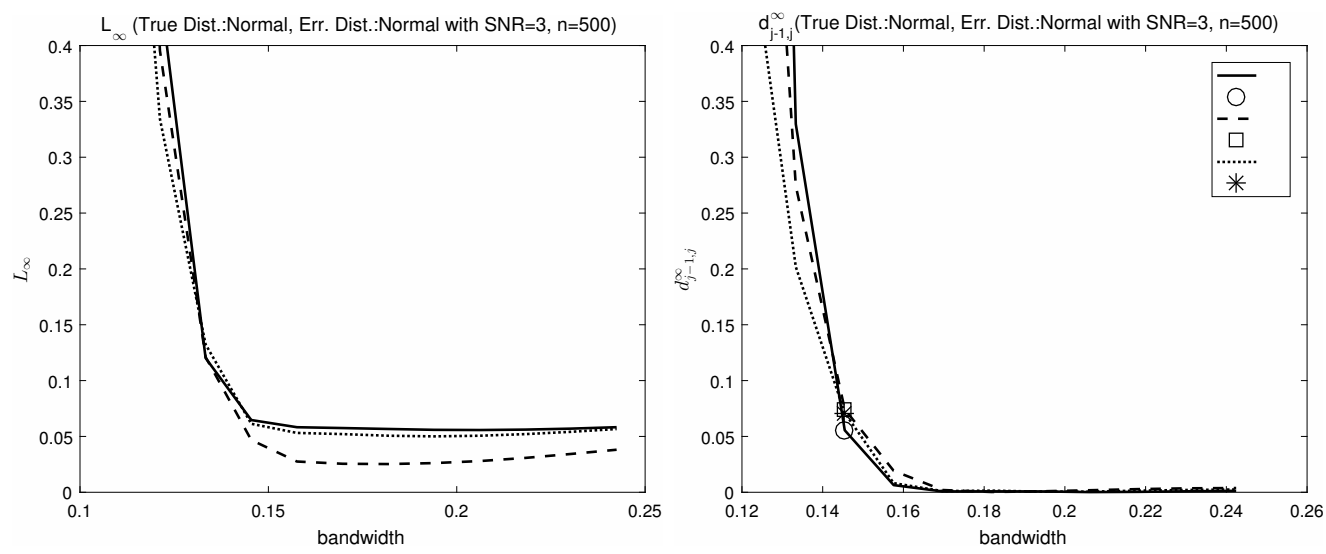

FiguRE 6.2. $L_{\infty}$ and $d_{j-1, j}^{\infty}$ distances under Normal error

size $n=100$ and the widths of the bands shrink substantially as the sample size increases from $n=100$ to $n=500$. They also show that the confidence bands with Laplace error gets narrower than those with Normal error as the sample size gets larger. This is consistent with our asymptotic theory since the convergence rates under Laplace and Normal errors are of polynomial and logarithmic order, respectively.

\section{Real Data EXAmple}

7.1. Data description. In this section, we apply the stochastic dominance test to the Korea Household Income and Expenditure Survey data to investigate welfare changes of different population sub-groups between 2006 and 2012. We use the data because the OECD report (2008) shows that, among OECD countries, Korea has the most significant variations in within-age group inequality and, compared to the inequality within the working age group, the relative inequality within the retirement age group is the worst. The data fit into our framework because it is well known that survey data are inherently affected by various sources of measurement errors; see Deaton (1997) and Bound, Brown and Mathiowetz (2000) for potential sources of 


\begin{tabular}{cccccccccc}
\hline \multirow{2}{*}{ Level } & \multirow{2}{*}{$n$} & Laplace Error & \multicolumn{5}{c}{ Normal Error } \\
& & True $X^{*}$ & SNR=1 & SNR=2 & SNR=3 & True $X^{*}$ & SNR=1 & SNR=2 & SNR=3 \\
\hline 0.80 & 100 & 0.803 & 0.771 & 0.832 & 0.829 & 0.792 & 0.745 & 0.769 & 0.817 \\
& 250 & 0.791 & 0.773 & 0.816 & 0.819 & 0.788 & 0.685 & 0.780 & 0.819 \\
& 500 & 0.812 & 0.791 & 0.812 & 0.833 & 0.790 & 0.664 & 0.780 & 0.805 \\
& 1000 & 0.793 & 0.794 & 0.800 & 0.802 & 0.797 & 0.638 & 0.764 & 0.803 \\
\hline 0.90 & 100 & 0.897 & 0.871 & 0.909 & 0.924 & 0.881 & 0.848 & 0.875 & 0.915 \\
& 250 & 0.899 & 0.868 & 0.909 & 0.907 & 0.903 & 0.796 & 0.872 & 0.920 \\
& 500 & 0.906 & 0.873 & 0.906 & 0.919 & 0.885 & 0.800 & 0.882 & 0.903 \\
& 1000 & 0.892 & 0.881 & 0.895 & 0.903 & 0.910 & 0.775 & 0.873 & 0.899 \\
\hline 0.95 & 100 & 0.948 & 0.922 & 0.958 & 0.962 & 0.939 & 0.911 & 0.938 & 0.951 \\
& 250 & 0.953 & 0.918 & 0.956 & 0.950 & 0.952 & 0.864 & 0.925 & 0.955 \\
& 500 & 0.956 & 0.923 & 0.958 & 0.957 & 0.936 & 0.867 & 0.940 & 0.948 \\
& 1000 & 0.947 & 0.929 & 0.945 & 0.961 & 0.951 & 0.858 & 0.925 & 0.946 \\
\hline
\end{tabular}

TABLE 1. Simulated uniform coverage probabilities for $F_{X^{*}}$ under Laplace and Normal errors.
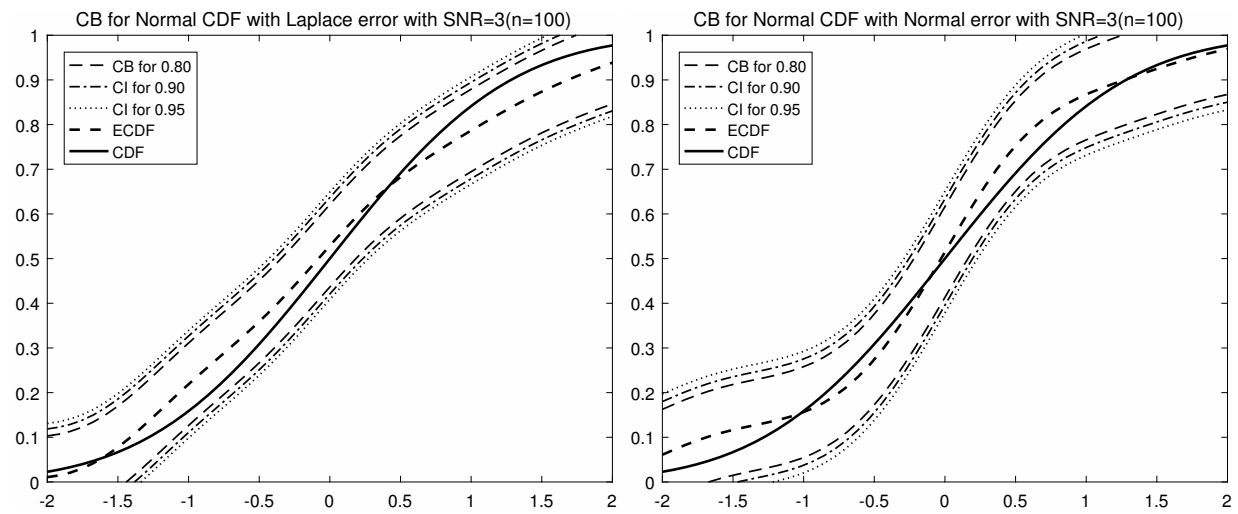

FiguRE 6.3. Uniform confidence bands under Laplace (left) and Normal (right) errors with $n=100$
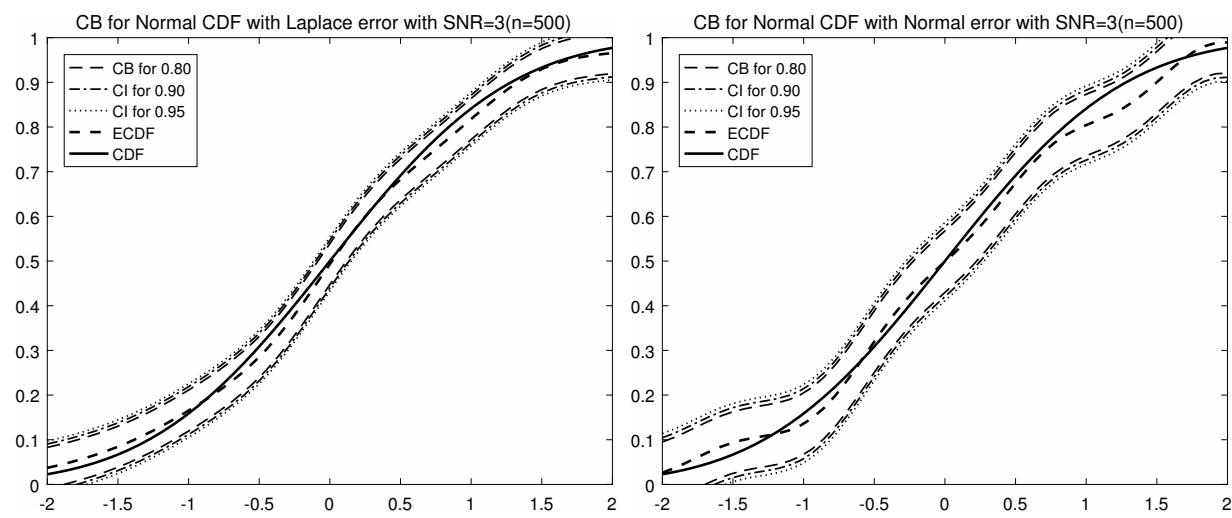

FiguRE 6.4. Uniform confidence bands under Laplace (left) and Normal (right) errors with $n=500$

measurement errors in household-based survey data. The survey reports incomes from various sources and consumption of goods and services for each household. We first compute the real 


\begin{tabular}{ccccc}
\hline Year & Age & Sample Size & Mean & S.D. \\
\hline 2006 & $25-45$ & 12045 & 1,650 & 910 \\
& $45-65$ & 8512 & 1,575 & 1,034 \\
& $60+$ & 4605 & 1,047 & 862 \\
& $65+$ & 3250 & 968 & 823 \\
& $70+$ & 2050 & 944 & 823 \\
\hline 2012 & $25-45$ & 8722 & 1,800 & 910 \\
& $45-65$ & 7653 & 1,814 & 1,106 \\
& $60+$ & 5166 & 1,105 & 934 \\
& $65+$ & 3700 & 974 & 879 \\
& $70+$ & 2439 & 891 & 857 \\
\hline
\end{tabular}

TABLE 2. Descriptive Statistics (Income unit: 1,000 won)

household disposable income by adding all incomes, public pension, social benefits and transfers, minus tax, public pension premium and social security fees, after adjusting for inflation using the 2010 consumer price index. We then obtain the individualized data by adjusting the total household disposable income using the square-root equivalization scale, which is a common practice to approximate individual welfare.

Table 2 shows the descriptive statistics for the data. It shows that average real incomes of individuals in all age group except those over 70 have increased from 2006 to 2012. Standard deviations of all incomes have also slightly increased over the same period. The results are consistent with the finding of OECD (2008). However, unless the income distributions are normal, comparison of only the first two moments is not sufficient to draw a conclusion on the uniform ordering of nonparametric income distributions that does not depend on a specific social welfare function. This motivates the use of a stochastic dominance criterion (see, e.g., Levy, 2016, and Whang, 2018).

7.2. Results. We consider two different null hypotheses for each age group: (i) The 2006 income distribution first-order stochastically dominates that the 2012 income distribution (abbreviated to 06 FSD 12) (ii) The 2012 income distribution first-order stochastically dominates the 2006 income distribution (abbreviated to 12 FSD 06). As a benchmark test, we consider the Barrett and Donald (2003, BD)'s test based on the observed incomes, neglecting the presence of measurement errors. We choose the bandwidth as in our simulation experiments and assume Laplace and normal measurement errors. The variance of measurement errors is determined so that the signal-to-noise ratio (SNR) is 1,2 , or $3 .^{6}$

\footnotetext{
${ }^{6}$ In practice, the error variance is generally not identified without repeated measurements or extraneous information. However, in the case of the CPS income survey data in the U.S., Bound and Krueger (1991) mentioned that "the error variance represents $27.6 \%$ of the total variance in CPS earnings for men and $8.9 \%$ for women." According to their remark, the signal-to-ratios (SNRs) are 0.9 for men and 2.35 for women. Since the SNR for our data might be different from those of the CPS data, the latter SNR values cannot be directly applied to our test. Instead, we opt for reporting the test results using SNRs in a "reasonable" range.
} 


\begin{tabular}{ccccccccc}
\hline \multirow{2}{*}{ Age } & \multirow{2}{*}{ Null Hypothesis } & \multirow{2}{*}{ BD } & \multicolumn{3}{c}{ Laplace Error } & \multicolumn{3}{c}{ Normal Error } \\
& & & SNR=1 & SNR=2 & SNR=3 & SNR=1 & SNR=2 & SNR=3 \\
\hline \multirow{2}{*}{25 -45 } & 06 FSD 12 & 0.000 & 0.000 & 0.000 & 0.000 & 0.000 & 0.000 & 0.000 \\
& 12 FSD 06 & 1.000 & 1.000 & 0.997 & 1.000 & 0.999 & 1.000 & 1.000 \\
\hline \multirow{2}{*}{$45-65$} & 06 FSD 12 & 0.000 & 0.000 & 0.000 & 0.000 & 0.000 & 0.000 & 0.000 \\
& 12 FSD 06 & 1.000 & 1.000 & 1.000 & 1.000 & 1.000 & 1.000 & 1.000 \\
\hline \multirow{2}{*}{$60+$} & 06 FSD 12 & 0.000 & 0.001 & 0.036 & 0.027 & 0.000 & 0.000 & 0.000 \\
& 12 FSD 06 & 0.039 & 0.995 & 0.002 & 0.001 & 0.369 & 0.309 & 0.233 \\
\hline \multirow{2}{*}{$65+$} & 06 FSD 12 & 0.353 & 0.302 & 0.410 & 0.645 & 0.089 & 0.180 & 0.217 \\
& 12 FSD 06 & 0.000 & 0.349 & 0.000 & 0.000 & 0.040 & 0.021 & 0.017 \\
\hline \multirow{2}{*}{$70+$} & 06 FSD 12 & 0.928 & 0.967 & 0.534 & 0.952 & 0.404 & 0.664 & 0.654 \\
& 12 FSD 06 & 0.000 & 0.002 & 0.000 & 0.000 & 0.000 & 0.000 & 0.000 \\
\hline
\end{tabular}

TABLE 3. Bootstrap P-values from BD and our tests

Table 3 reports the bootstrap p-values of the tests. The BD test implies that, for age groups 25-45 and 45-65, the 2012 income significantly dominates the 2006 income and, for age group $60+$, there appears to be no dominance relationship (i.e. the two distributions cross), while for age group 70+ the 2006 income dominates the 2012 income. Similar results hold when we apply our test assuming Laplace measurement errors with relatively large SNR $(=2,3)$. However, when the $\mathrm{SNR}$ is small $(\mathrm{SNR}=1)$, or the error distribution is normal, our test shows that, for age group $60+$, there is a significant evidence that the 2012 income dominates the 2006 income. Similarly, for age group $65+$, the test results vary across different SNRs. This confirms that the presence of measurement errors in the observed data might lead to different conclusions on the stochastic dominance relationship of the underlying true distributions. Without additional information, it is hard to draw a definite conclusion on the stochastic dominance relationship, but we believe that our results can serve as a pilot study for a more systematic future investigation of the dynamics of the income distributions when they are contaminated with measurement errors. 


\section{Appendix A. Proofs of theorems}

Notation: Hereafter, let $P^{\#}$ and $E^{\#}$ be the conditional probability and expectation under the bootstrap distribution given $\left\{X_{i}\right\}_{i=1}^{n}$, respectively. Also, denote

$$
\begin{aligned}
\overline{\mathbb{L}}(u) & =\frac{1}{\pi} \int_{0}^{1} \frac{\sin (\omega u)}{\omega} \frac{K^{\mathrm{ft}}(\omega)}{f_{\epsilon}^{\mathrm{ft}}(\omega / h)} \mathbb{I}\left\{|\omega| \geq h \omega_{0}\right\} d \omega, \\
\mathcal{G}_{n}(t) & =r(h) \int \overline{\mathbb{L}}\left(\frac{t-a}{h}\right) f_{X}(a)^{1 / 2} d W(a), \\
p_{\varepsilon}\left(\mathcal{G}_{n}\right) & =\sup _{x} P\left\{\left|\sup _{t \in \mathcal{T}} \mathcal{G}_{n}(t)-x\right| \leq \varepsilon\right\},
\end{aligned}
$$

where $W$ is a (two-sided) Wiener process on $\mathbb{R}, f_{X}$ is the pdf of $X$, and

$$
r(h)=\left\{\begin{array}{cc}
h^{\beta-\frac{1}{2}} & \text { under Assumption OS } \\
1 / \varsigma(h) & \text { under Assumption SS }
\end{array},\right.
$$

with $\varsigma(h)$ defined in eq. (2.12) of the paper. Note that analogous to $\overline{\mathbb{K}}$ (defined in Assumptions OS (ii) and SS (ii)), $\overline{\mathbb{L}}$ is considered as a trimmed version of $\mathbb{L}$. Due to the trimming, properties of the Fourier transform guarantee $\overline{\mathbb{L}} \in L_{2}(\mathbb{R})$ for each $h$ under the assumption $f_{\epsilon}^{\mathrm{ft}} \neq 0$, and this guarantees existence of the stochastic integral in the definition of $\mathcal{G}_{n}$.

Also, for any $a \in(0,1)$, let $c_{a}$ denote the constant such that $\sqrt{n} h^{\beta-\frac{1}{2}} c_{a}$ is the $(1-a)$-th quantile of $\sup _{t \in \mathcal{T}}\left|\mathcal{G}_{n}(t)\right|$.

A.1. Proof of Theorem 1. Since the proof is similar, we only present the proof for the ordinary smooth case. Define

$$
\hat{D}_{n}^{\#}(t)=\sqrt{n} h^{\beta-1 / 2}\left\{\hat{F}_{X^{*}}^{\#}(t)-\hat{F}_{X^{*}}(t)\right\}, \quad \tilde{D}_{n}^{\#}(t)=\sqrt{n} h^{\beta-1 / 2}\left\{\tilde{F}_{X^{*}}^{\#}(t)-\tilde{F}_{X^{*}}(t)\right\}
$$

We first show that there exist $c, C>0$ such that

$$
P^{\#}\left\{\sup _{t \in \mathcal{T}}\left|\tilde{D}_{n}^{\#}(t)-\hat{D}_{n}^{\#}(t)\right| \geq C n^{-c}\right\}=o_{p}(1) .
$$

By Lemma 2, it is enough for (A.1) to guarantee that there exist $c, C>0$ satisfying

$$
P^{\#}\left\{\sqrt{n} h^{\beta-1 / 2} \sup _{t \in \mathcal{T}}\left|\tilde{F}_{X^{*}}^{\#}(t)-\hat{F}_{X^{*}}^{\#}(t)\right| \geq C n^{-c}\right\}=o_{p}(1) .
$$


Let $\hat{f}_{X}^{\mathrm{ft \#}}(\omega)=N^{-1} \sum_{i, j} e^{\mathrm{i} \omega X_{i, j}^{\#}}$ be the bootstrap counterpart of the empirical characteristic function $\hat{f}_{X}^{\mathrm{ft}}(\omega)=N^{-1} \sum_{i, j} e^{\mathrm{i} \omega X_{i, j}}, \xi=\left(f_{\epsilon}^{\mathrm{ft}}\right)^{2}$, and $\hat{\xi}=\left(\hat{f}_{\epsilon}^{\mathrm{ft}}\right)^{2}$. Note that

$$
\begin{aligned}
\tilde{F}_{X^{*}}^{\#}(t)-\hat{F}_{X^{*}}^{\#}(t)= & \frac{1}{2 \pi} \int_{-1 / h}^{1 / h} \frac{\operatorname{Im}\left\{e^{-\mathrm{i} \omega t}\left\{\hat{f}_{X}^{\mathrm{ft} \#}(\omega)-\hat{f}_{X}^{\mathrm{ft}}(\omega)\right\}\right\}}{-\omega}\left\{\hat{\xi}(\omega)^{-1 / 2}-\xi(\omega)^{-1 / 2}\right\} K^{\mathrm{ft}}(h \omega) d \omega \\
& +\frac{1}{2 \pi} \int_{-1 / h}^{1 / h} \frac{\operatorname{Im}\left\{e^{-\mathrm{i} \omega t} \hat{f}_{X}^{\mathrm{ft}}(\omega)\right\}}{-\omega}\left\{\hat{\xi}(\omega)^{-1 / 2}-\xi(\omega)^{-1 / 2}\right\} K^{\mathrm{ft}}(h \omega) d \omega \\
= & C_{1 n}(t)+C_{2 n}(t) .
\end{aligned}
$$

The second term $C_{2 n}(t)$ equals to $\tilde{F}_{X^{*}}(t)-\hat{F}_{X^{*}}(t)$ whose bound is given in Lemma 2 . Thus, we only need to consider the first term $C_{1 n}(t)$. By expanding the expectations, it can be shown

$$
E^{\#}\left[\int_{\omega_{0}}^{1 / h} \omega^{-a}\left|\hat{f}_{X}^{\mathrm{ft \#}}(\omega)-\hat{f}_{X}^{\mathrm{ft}}(\omega)\right|^{2} d \omega\right]=O_{p}\left(\left(n h^{1-a}\right)^{-1}\right),
$$

for all $a<1$, and analogous arguments as in the proof of Lemma 2 yield $\sup _{t \in \mathcal{T}}\left|C_{1 n}(t)\right|=$ $O_{p \#}\left(\left(n h^{3 \beta}\right)^{-1}\right)$ with probability approaching one. Therefore, by paralleling the arguments in the proof of Lemma 2, we obtain (A.1).

We now proceed by verifying the conditions in the proof of Theorem 3. Lemmas 2 and 6 ensure existence of a sequence $\epsilon_{n}=O\left(n^{-c}\right)$ with some $c>0$ such that

$$
P\left\{\sup _{t \in \mathcal{T}}\left|\sqrt{n} h^{\beta-1 / 2}\left\{\tilde{F}_{X}(t)-F_{X}(t)\right\}-\mathcal{G}_{n}(t)\right|>\epsilon_{n}\right\}=o_{p}(1) .
$$

Furthermore by Lemma 7, combined with (A.1), we have that

$$
P^{\#}\left\{\sup _{t \in \mathcal{T}}\left|\sqrt{n} h^{\beta-1 / 2}\left\{\tilde{F}_{X^{*}}^{\#}(t)-\tilde{F}_{X^{*}}(t)\right\}-\tilde{\mathcal{G}}_{n}(t)\right|>\epsilon_{n}\right\}=o_{p}(1) .
$$

Therefore, by (A.2) and (A.3), the conclusion follows by paralleling the arguments in the proof of Theorem 3 .

A.2. Proof of Theorem 2. Since the proof is similar, we only present the proof for the ordinary smooth case. Define $\bar{F}_{X^{*}}^{A}(t)=\frac{1}{2}-\frac{1}{m} \sum_{i=1}^{m} \frac{1}{2} \sum_{j=1}^{2} \mathbb{L}^{A}\left(\frac{t-X_{i, j}}{h}\right), \bar{F}_{X^{*}}^{A \#}(t)=\frac{1}{2}-\frac{1}{m} \sum_{i=1}^{m} \frac{1}{2} \sum_{j=1}^{2} \mathbb{L}^{A}\left(\frac{t-X_{i, j}^{\#}}{h}\right)$, and

$$
\check{D}_{n}^{A \#}(t)=\sqrt{m} h^{\beta-1 / 2}\left\{\check{F}_{X^{*}}^{A \#}(t)-\check{F}_{X^{*}}^{A}(t)\right\}, \quad \bar{D}_{n}^{A \#}(t)=\sqrt{m} h^{\beta-1 / 2}\left\{\bar{F}_{X^{*}}^{A \#}(t)-\bar{F}_{X^{*}}^{A}(t)\right\}
$$

We first show that there exist $c, C>0$ such that

$$
P^{\#}\left\{\sup _{t \in \mathcal{T}}\left|\bar{D}_{n}^{A \#}(t)-\check{D}_{n}^{A \#}(t)\right| \geq C n^{-c}\right\}=o_{p}(1) .
$$


By Lemma 3, it is enough for (A.4) to guarantee that there exist $c, C>0$ satisfying

$$
P^{\#}\left\{\sqrt{m} h^{\beta-1 / 2} \sup _{t \in \mathcal{T}}\left|\bar{F}_{X^{*}}^{A \#}(t)-\check{F}_{X^{*}}^{A \#}(t)\right| \geq C n^{-c}\right\}=o_{p}(1) .
$$

Let $\hat{f}_{X, m}^{\mathrm{ft} \#}(\omega)=m^{-1} \sum_{i=1}^{m} \frac{1}{2} \sum_{j=1}^{2} e^{\mathrm{i} \omega X_{i, j}^{\#}}$ be the bootstrap counterpart of the empirical characteristic function $\hat{f}_{X, m}^{\mathrm{ft}}(\omega)=m^{-1} \sum_{i=1}^{m} \frac{1}{2} \sum_{j=1}^{2} e^{\mathrm{i} \omega X_{i, j}}$. Note that

$$
\begin{aligned}
\bar{F}_{X^{*}}^{A \#}(t)-\check{F}_{X^{*}}^{A \#}(t)= & \frac{1}{2 \pi} \int_{-1 / h}^{1 / h} \frac{\operatorname{Im}\left\{e^{-\mathrm{i} \omega t}\left\{\hat{f}_{X, m}^{\mathrm{ft} \#}(\omega)-\hat{f}_{X, m}^{\mathrm{ft}}(\omega)\right\}\right\}}{-\omega}\left\{\check{f}_{\epsilon}^{\mathrm{ft}}(\omega)^{-1}-f_{\epsilon}^{\mathrm{ft}}(\omega)^{-1}\right\} K^{\mathrm{ft}}(h \omega) d \omega \\
& +\frac{1}{2 \pi} \int_{-1 / h}^{1 / h} \frac{\operatorname{Im}\left\{e^{-\mathrm{i} \omega t} \hat{f}_{X, m}^{\mathrm{ft}}(\omega)\right\}}{-\omega}\left\{\check{f}_{\epsilon}^{\mathrm{ft}}(\omega)^{-1}-f_{\epsilon}^{\mathrm{ft}}(\omega)^{-1}\right\} K^{\mathrm{ft}}(h \omega) d \omega \\
= & C_{1 n}^{A}(t)+C_{2 n}^{A}(t) .
\end{aligned}
$$

Then the bootstrap version of Lemma 3 implies $\sup _{t \in \mathcal{T}}\left|C_{2 n}^{A}(t)\right|=o_{p^{\#}}\left(n^{-c}\right)$ for some $c>0$. By expanding the expectations, it can be shown

$$
E^{\#}\left[\int_{\omega_{0}}^{1 / h} \omega^{-a}\left|\hat{f}_{X, m}^{\mathrm{ft} \#}(\omega)-\hat{f}_{X, m}^{\mathrm{ft}}(\omega)\right|^{2} d \omega\right]=O_{p}\left(\left(m h^{1-a}\right)^{-1}\right),
$$

for all $a<1$, and analogous arguments as in the proof of Lemma 3 yield $\sup _{t \in \mathcal{T}}\left|C_{1 n}^{A}(t)\right|=$ $O_{p \#}\left(\left(m h^{3 \beta}\right)^{-1}\right)$ with probability approaching one. Therefore, by paralleling the arguments in the proof of Lemma 3, we obtain (A.4).

The rest of the proof is similar as that of Theorem 1: verify the conditions in the proof of Theorem 3 by replacing $\mathbb{L}$ with $\mathbb{L}^{A}$, and note that $\mathbb{L}^{A}(u)=\int_{-\infty}^{u} \mathbb{K}(v) d v$ in view of the results from Dattner, Goldenshluger and Juditsky (2011).

A.3. Proof of Theorem 3. We only prove the statement under Assumption OS (i.e., the ordinary smooth case). The statement under Assumption SS is shown by a similar argument using Lemmas 9-11.

First, we prove

$$
P\left\{\sqrt{n} h^{\beta-\frac{1}{2}} \tilde{c}_{\alpha}>\sqrt{n} h^{\beta-\frac{1}{2}} c_{\alpha+\delta_{1 n}}-\epsilon_{1 n}\right\} \geq 1-\delta_{2 n},
$$

for some $\epsilon_{1 n}, \delta_{1 n}, \delta_{2 n}=O\left(n^{-c}\right)$ with $c>0$. Lemma 7 implies that with probability greater than $1-\delta_{2 n}$,

$$
\begin{aligned}
1-\alpha & =P^{\#}\left\{\sqrt{n} h^{\beta-\frac{1}{2}} \sup _{t \in \mathcal{T}}\left|\tilde{F}_{X^{*}}^{\#}(t)-\tilde{F}_{X^{*}}(t)\right| \leq \sqrt{n} h^{\beta-\frac{1}{2}} \tilde{c}_{\alpha}\right\} \\
& \leq P^{\#}\left\{\sup _{t \in \mathcal{T}}\left|\tilde{\mathcal{G}}_{n}(t)\right| \leq \sqrt{n} h^{\beta-\frac{1}{2}} \tilde{c}_{\alpha}+\epsilon_{1 n}\right\}+\delta_{1 n},
\end{aligned}
$$

for some $\epsilon_{1 n}, \delta_{1 n}, \delta_{2 n}=O\left(n^{-c}\right)$ with $c>0$, where $\tilde{\mathcal{G}}_{n}$ has the same distribution as $\mathcal{G}_{n}$ under $P^{\#}$. Since $\sqrt{n} h^{\beta-\frac{1}{2}} c_{a}$ is also the $(1-a)$-th quantile of $\sup _{t \in \mathcal{T}}\left|\tilde{\mathcal{G}}_{n}(t)\right|$ under $P^{\#}$, the above inequality 
implies

$$
P^{\#}\left\{\sup _{t \in \mathcal{T}}\left|\tilde{\mathcal{G}}_{n}(t)\right| \leq \sqrt{n} h^{\beta-\frac{1}{2}} c_{\alpha+\delta_{1 n}}\right\} \leq P^{\#}\left\{\sup _{t \in \mathcal{T}}\left|\tilde{\mathcal{G}}_{n}(t)\right| \leq \sqrt{n} h^{\beta-\frac{1}{2}} \tilde{c}_{\alpha}+\epsilon_{1 n}\right\},
$$

with probability greater than $1-\delta_{2 n}$. Thus, we obtain (A.5).

The main result is thus obtained from the following sequence of inequalities

$$
\begin{aligned}
P\left\{T_{n} \leq \tilde{c}_{\alpha}\right\} & \geq P\left\{\sup _{t \in \mathcal{T}}\left|\mathcal{G}_{n}(t)\right| \leq \sqrt{n} h^{\beta-\frac{1}{2}} \tilde{c}_{\alpha}-\epsilon_{n}\right\}-\delta_{n} \\
& \geq P\left\{\sup _{t \in \mathcal{T}}\left|\mathcal{G}_{n}(t)\right| \leq \sqrt{n} h^{\beta-\frac{1}{2}} c_{\alpha+\delta_{1 n}}-\epsilon_{1 n}-\epsilon_{n}\right\}-\delta_{n}-\delta_{2 n} \\
& \geq P\left\{\sup _{t \in \mathcal{T}}\left|\mathcal{G}_{n}(t)\right| \leq \sqrt{n} h^{\beta-\frac{1}{2}} c_{\alpha+\delta_{1 n}}\right\}-2 p_{\bar{\epsilon}_{n}}\left(\mathcal{G}_{n}\right)-\delta_{n}-\delta_{2 n} \\
& =1-\alpha-\delta_{1 n}-2 p_{\bar{\epsilon}_{n}}\left(\mathcal{G}_{n}\right)-\delta_{n}-\delta_{2 n} \\
& \geq 1-\alpha-\delta_{1 n}-M \bar{\epsilon}_{n} \sqrt{\log (1 / h)}-\delta_{n}-\delta_{2 n},
\end{aligned}
$$

where the first inequality follows from Lemma 6, the second inequality follows from (A.5), the third inequality follows from the definitions of $\bar{\epsilon}_{n}=\epsilon_{1 n}+\epsilon_{n}$ and $p_{\varepsilon}\left(\mathcal{G}_{n}\right)$, along with the fact $\mathcal{G}_{n}$ and $-\mathcal{G}_{n}$ have the same distribution (which ensures $p_{\varepsilon}\left(\left|\mathcal{G}_{n}\right|\right) \leq 2 p_{\varepsilon}\left(\mathcal{G}_{n}\right)$ ), the equality follows from the definition that $\sqrt{n} h^{\beta-\frac{1}{2}} c_{\alpha+\delta_{1 n}}$ is the $\left(1-\alpha-\delta_{1 n}\right)$-th quantile of $\sup _{t \in \mathcal{T}}\left|\mathcal{G}_{n}(t)\right|$, and the last inequality follows from Lemma 8. Therefore, letting $\delta_{3 n}=\delta_{1 n}+M \bar{\epsilon}_{n} \sqrt{\log (1 / h)}+\delta_{n}+\delta_{2 n}$, we have

$$
P\left\{T_{n} \leq \tilde{c}_{\alpha}\right\} \geq 1-\alpha-\delta_{3 n}
$$

Since $\delta_{n}, \delta_{1 n}, \delta_{2 n}, \bar{\epsilon}_{n}$ are all positive sequences of order $O\left(n^{-a}\right)$ with some $a>0$ and $\sqrt{\log (1 / h)}$ is a log-rate, we obtain (4.2).

A.4. Proof of Theorem 5. We only prove the theorem under Assumption OS (i.e., the ordinary smooth case). The proof under Assumption SS follows by a similar argument using Lemmas $9-11$.

We make the following preliminary observations. First, by the techniques employed in Lemmas $6-8$, we can show ${ }^{7}$

$$
\sup _{t \in \mathcal{H}}\left|\tilde{f}_{X^{*}}(t)-f_{X^{*}}(t)\right|=O_{p}\left(n^{-c}\right) .
$$

Next by Dattner, Reiß and Trabs (2016, Proposition 2.1), $\left\|\tilde{f}_{X^{*}}\right\|_{1}<\infty$ and $\int_{-\infty}^{\infty} \tilde{f}_{X^{*}}(t) d t=1$ under Assumption C. Thus, we have $\tilde{F}_{X^{*}}(t)=\int_{-\infty}^{t} \tilde{f}_{X^{*}}(v) d v$ or equivalently $\tilde{F}_{X^{*}}^{\prime}(t)=\tilde{f}_{X^{*}}(t)$. The latter ensures $\tilde{F}_{X^{*}}$ is continuous.

\footnotetext{
${ }^{7}$ An analogous result applies for the super smooth case by Lemmas 9-11 with the rate replaced by $O_{p}\left((\log n)^{-c}\right)$ for some $c>1$ under the assumption $\gamma>\lambda$ and an MSE optimal bandwidth choice.
} 
We now show that ${ }^{8}$

$$
\sup _{u \in\left[u_{1}, u_{2}\right]}|\tilde{Q}(u)-Q(u)|=o_{p}\left(n^{-c_{1}}\right),
$$

for some $c_{1}>0$. By Hall and Lahiri (2008, Theorem 3.7), $\tilde{Q}(u)$ converges to $Q(u)$ for each $u \in\left[u_{1}, u_{2}\right]$. Now $Q_{n}(u)$ is monotone at each $n$ by construction while $Q(u)$ is continuous by Assumption Q (i). Hence we can modify the proof of the Glivenko-Cantelli theorem (see, Billingsley, 1995, p. 233), to strengthen the pointwise consistency to a uniform one, i.e.,

$$
\sup _{u \in\left[u_{1}, u_{2}\right]}|\tilde{Q}(u)-Q(u)|=o_{p}(1)
$$

(see also, Bassett and Koenker, 1986, Theorem 3.1). As $\hat{F}_{X^{*}}$ is continuous, it follows that $\tilde{F}_{X^{*}}(\tilde{Q}(u))=u$ for all $0<u<1$. Consequently,

$$
\tilde{F}_{X^{*}}(\tilde{Q}(u))=F_{X^{*}}(Q(u))=F_{X^{*}}(\tilde{Q}(u))-f_{X^{*}}(\bar{Q}(u))(\tilde{Q}(u)-Q(u)),
$$

for some $\bar{Q}(u)$ such that $|\bar{Q}(u)-Q(u)| \leq|\tilde{Q}(u)-Q(u)|$, and we obtain

$$
\sup _{u \in\left[u_{1}, u_{2}\right]}|\tilde{Q}(u)-Q(u)| \leq\left(\inf _{u \in\left[u_{1}, u_{2}\right]}\left|f_{X^{*}}(\bar{Q}(u))\right|\right)^{-1} \sup _{u \in\left[u_{1}, u_{2}\right]}\left|\tilde{F}_{X^{*}}(\tilde{Q}(u))-F_{X^{*}}(\tilde{Q}(u))\right|
$$

By (A.8) and Assumption Q (i) $\left(\inf _{x \in \mathcal{H}} f_{X^{*}}(x)>0\right)$, we can verify $\inf _{u \in\left[u_{1}, u_{2}\right]}\left|f_{X^{*}}(\bar{Q}(u))\right|>0$ with probability approaching one. Furthermore, we have

$$
\sup _{u \in\left[u_{1}, u_{2}\right]}\left|\tilde{F}_{X^{*}}(\tilde{Q}(u))-F_{X^{*}}(\tilde{Q}(u))\right| \leq n^{-\frac{1}{2}} h^{-\beta+\frac{1}{2}} \sup _{t \in \mathcal{H}}\left|\mathcal{G}_{n}(t)\right|+o_{p}(1)=O_{p}\left(\left(\frac{\log (1 / h)}{n h^{2 \beta-1}}\right)^{1 / 2}\right),
$$

where the inequality follows from Lemma 6 after employing the fact $\left\{\tilde{Q}(u): u \in\left[u_{1}, u_{2}\right]\right\} \subset \mathcal{H}$ with probability approaching one due to Assumption Q (i) and (A.8). The equality follows from $E\left[\sup _{t \in \mathcal{H}}\left|\mathcal{G}_{n}(t)\right|\right]=O(\sqrt{\log (1 / h)})$ (by the proof of Lemma 8). Combining these results, we obtain (A.7) under Assumptions OS (iii) and B (ii).

We now proceed to the main part of the proof. Noting that $\tilde{Q}(u)-Q(u)=f_{X^{*}}(\bar{Q}(u))^{-1}\left\{\tilde{F}_{X^{*}}(\tilde{Q}(u))-\right.$ $\left.F_{X^{*}}(\tilde{Q}(u))\right\}$, we have

$$
\begin{aligned}
& P\left\{\tilde{Q}(u)-\frac{\tilde{c}_{\alpha}}{\tilde{f}_{X^{*}}(\tilde{Q}(u))} \leq Q(u) \leq \tilde{Q}(u)+\frac{\tilde{c}_{\alpha}}{\tilde{f}_{X^{*}}(\tilde{Q}(u))} \quad \text { for all } u \in\left[u_{1}, u_{2}\right]\right\} \\
= & P\left\{\sup _{u \in\left[u_{1}, u_{2}\right]}\left|\tilde{f}_{X^{*}}(\tilde{Q}(u))\{\tilde{Q}(u)-Q(u)\}\right| \leq \tilde{c}_{\alpha}\right\} \geq P\left\{\sup _{t \in \mathcal{H}}\left|\tilde{F}_{X^{*}}(t)-F_{X^{*}}(t)\right| \leq \tilde{c}_{\alpha}\left(1-\Delta_{n}\right)\right\}-o(1),
\end{aligned}
$$

where $\Delta_{n}=\sup _{u \in\left[u_{1}, u_{2}\right]}\left|\frac{\tilde{f}_{X^{*}}(\tilde{Q}(u))-f_{X^{*}}(\bar{Q}(u))}{\tilde{f}_{X^{*}}(\tilde{Q}(u))}\right|$ and the inequality follows from the fact $P\left\{\left\{\tilde{Q}(u): u \in\left[u_{1}, u_{2}\right]\right\} \subset \mathcal{H}\right\} \rightarrow 1$ by Assumption Q (i) and (A.7). Also note that $\Delta_{n}=$ $O_{p}\left(n^{-c}\right)$ by Assumption Q (i)-(ii), (A.6), and (A.7). We now have the following sequence of

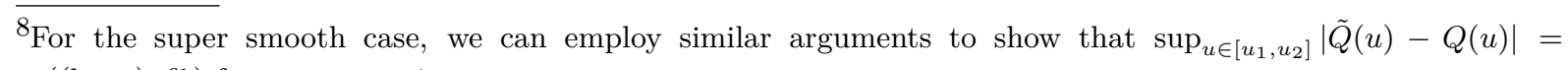
$o_{p}\left((\log n)^{-c_{1}}\right)$ for some $c_{1}>1$. 
inequalities

$$
\begin{aligned}
& P\left\{\sup _{t \in \mathcal{H}}\left|\tilde{F}_{X^{*}}(t)-F_{X^{*}}(t)\right| \leq \tilde{c}_{\alpha}\left(1-\Delta_{n}\right)\right\} \geq P\left\{\sup _{t \in \mathcal{H}}\left|\mathcal{G}_{n}(t)\right| \leq \sqrt{n} h^{\beta-\frac{1}{2}} \tilde{c}_{\alpha}\left(1-\Delta_{n}\right)-\epsilon_{n}\right\}-\delta_{n} \\
\geq & P\left\{\sup _{t \in \mathcal{H}}\left|\mathcal{G}_{n}(t)\right| \leq\left(\sqrt{n} h^{\beta-\frac{1}{2}} c_{\alpha+\delta_{1 n}}-\epsilon_{1 n}\right)\left(1-\Delta_{n}\right)-\epsilon_{n}\right\}-\delta_{n}-\delta_{2 n} \\
\geq & P\left\{\sup _{t \in \mathcal{H}}\left|\mathcal{G}_{n}(t)\right| \leq \sqrt{n} h^{\beta-\frac{1}{2}} c_{\alpha+\delta_{1 n}}\right\}-2 p_{\bar{\epsilon}_{n}}\left(\mathcal{G}_{n}\right)-\delta_{n}-\delta_{2 n} \geq 1-\alpha-\delta_{1 n}-\delta_{n}-\delta_{2 n}-2 p_{\bar{\epsilon}_{n}}\left(\mathcal{G}_{n}\right),
\end{aligned}
$$

where the first inequality follows from Lemma 6 , the second inequality can be derived by Lemma 7 and a similar argument in the proof of Theorem 1, the third inequality follows from the definitions of $\bar{\epsilon}_{n}=\epsilon_{n}+\epsilon_{1 n}\left(1-\Delta_{n}\right)+\sqrt{n} h^{\beta-\frac{1}{2}} c_{\alpha+\delta_{1 n}} \Delta_{n}$ and the concentration function. Note that Lemma 8 implies $p_{\bar{\epsilon}_{n}}\left(\mathcal{G}_{n}\right) \leq C \bar{\epsilon}_{n} \sqrt{\log n}$. Recalling that $\sqrt{n} h^{\beta-\frac{1}{2}} c_{\alpha+\delta_{1 n}}$ is the $\left(\alpha+\delta_{1 n}\right)$-th quantile of $\sup _{t \in \mathcal{H}}\left|\mathcal{G}_{n}(t)\right|$, by Chernozhukov, Chetverikov and Kato (2014, Lemma B1),

$$
\sqrt{n} h^{\beta-\frac{1}{2}} c_{\alpha+\delta_{1 n}} \leq E\left[\sup _{t \in \mathcal{H}}\left|\mathcal{G}_{n}(t)\right|\right]+\sqrt{2\left|\log \left(\alpha+\delta_{1 n}\right)\right|} .
$$

Since $E\left[\sup _{t \in \mathcal{H}}\left|\mathcal{G}_{n}(t)\right|\right]=O(\sqrt{\log (1 / h)})$, this implies $\sqrt{n} h^{\beta-\frac{1}{2}} c_{\alpha+\delta_{1 n}}=O(\sqrt{\log n})$ under Assumptions OS (iii) and B (ii). By the above and the rates of $\epsilon_{n}, \epsilon_{1 n}$, it follows $p_{\bar{\epsilon}_{n}}\left(\mathcal{G}_{n}\right)=O_{p}\left(n^{-c_{2}}\right)$ for some $c_{2}>0$. Furthermore, by Lemmas 6 and $7, \delta_{n}, \delta_{1 n}$, and $\delta_{2 n}$ are also $O\left(n^{-c_{3}}\right)$ for some $c_{3}>0$. Combining these results, the conclusion follows.

A.5. Proof of Theorem 6. We shall assume for simplicity that $f_{\epsilon}=f_{\delta}$, and consequently that the bandwidth choices for both estimators are the same. We only prove for the case of ordinary smooth error density as the proof for super-smooth density follows by the same arguments. Assume that the smoothness parameter in the former case is $\beta$. Let

$$
\mathcal{G}_{n, m}^{D}(t)=h^{\beta-1 / 2}\left\{\int \overline{\mathbb{L}}\left(\frac{t-a}{h}\right) f_{X}(a)^{1 / 2} d W_{1}(a)-\sqrt{\frac{n}{m}} \int \overline{\mathbb{L}}\left(\frac{t-a}{h}\right) f_{Y}(a)^{1 / 2} d W_{2}(a)\right\},
$$

where $W_{1}$ and $W_{2}$ are two independent (two-sided) Wiener processes on $\mathbb{R}$ (for $f_{\epsilon} \neq f_{\delta}$ or unequal bandwidths, the $\overline{\mathbb{L}}$ functions in the above integrals would also be different). Also define

$$
\begin{aligned}
& \Psi_{n, m}(t)=\left\{\tilde{F}_{X^{*}}(t)-F_{X^{*}}(t)\right\}-\left\{\tilde{F}_{Y^{*}}(t)-F_{Y^{*}}(t)\right\} \\
& \Psi_{n, m}^{\#}(t)=\left\{\tilde{F}_{X^{*}}^{\#}(t)-\tilde{F}_{X^{*}}(t)\right\}-\left\{\tilde{F}_{Y^{*}}^{\#}(t)-\tilde{F}_{Y^{*}}(t)\right\}
\end{aligned}
$$

A.5.1. Proof of (i). Since the samples $\left\{X_{i}\right\}_{i=1}^{n}$ and $\left\{Y_{i}\right\}_{i=1}^{m}$ are independent of each other, by the arguments of Lemmas (6)-(8), we can show the following: For some sequences $\epsilon_{n}, \delta_{n}=O\left(n^{-c}\right)$,

$$
P\left\{\sup _{t \in \mathcal{T}}\left|\sqrt{n} h^{\beta-1 / 2} \Psi_{n, m}(t)-\mathcal{G}_{n, m}^{D}(t)\right|>\epsilon_{n}\right\}<\delta_{n} .
$$


Furthermore with probability greater than $1-\delta_{2 n}, \delta_{2 n}=O\left(n^{-c}\right)$, there exist sequences $\epsilon_{1 n}, \delta_{1 n}=$ $O\left(n^{-c}\right)$ such that

$$
P^{\#}\left\{\sup _{t \in \mathcal{T}}\left|\sqrt{n} h^{\beta-1 / 2} \Psi_{n, m}^{\#}(t)-\tilde{\mathcal{G}}_{n, m}^{D \#}(t)\right|>\epsilon_{1 n}\right\}<\delta_{1 n},
$$

where $\tilde{\mathcal{G}}_{n, m}^{D \#}$ is a tight Gaussian process with the same distribution as $\mathcal{G}_{n, m}^{D}$ under $P^{\#}$. Finally it also holds that

$$
p_{\epsilon_{n}}\left(\mathcal{G}_{n, m}^{D}\right) \leq M \epsilon_{n} \sqrt{\log (1 / h)}
$$

for any sequence $\epsilon_{n}=O\left(n^{-c}\right)$ and some $M<\infty$. Now

$$
P\left\{D_{n, m} \leq \hat{c}_{\alpha}^{D}\right\} \geq P\left\{\sup _{t \in \mathcal{T}} \Psi_{n, m}(t)-\sup _{t}\left\{F_{X^{*}}(t)-F_{Y^{*}}(t)\right\} \leq \hat{c}_{\alpha}^{D}\right\} \geq P\left\{\sup _{t \in \mathcal{T}} \Psi_{n, m}(t) \leq \hat{c}_{\alpha}^{D}\right\},
$$

where the last equality follows from $\sup _{t}\left\{F_{X^{*}}(t)-F_{Y^{*}}(t)\right\} \leq 0$ under $H_{0}$. Using equations (A.9)-(A.11), by paralleling the arguments in the proof of Theorem 1, we can show that

$$
P\left\{\sup _{t \in \mathcal{T}} \Psi_{n, m}(t) \leq \hat{c}_{\alpha}^{D}\right\} \geq 1-\alpha-\varrho_{n, m}
$$

Hence the claim follows immediately.

A.5.2. Proof of (ii). It is enough to show that $\rho_{n, m}$ does not depend on $P \in \mathcal{P}_{0}$. To this end, it is enough to show uniform validity of equations (A.9)-(A.11). Since these equations are essentially two-sample counterparts of Lemmas (6)-(8), it suffices to check uniform validity of the latter.

Note that for Lemma (6), uniformity of the bias term follows by the argument in Hall and Lahiri (2008, Theorem 3.2) using the uniform version of the Sobolev condition (i.e. the constants $M_{X}$ and $M_{Y}$ do not depend on $\left.\left(F_{X^{*}}, F_{Y^{*}}\right)\right)$. For the stochastic term, the constants appearing in the KMT coupling in the proof of Lemma (6) are universal, and constants and sequences in other parts do not depend on $P \in \mathcal{P}_{0}$. Thus, $\delta_{n}$ in Lemma (6) does not depend on $P \in \mathcal{P}_{0}$. Similarly, uniformity of Lemma (7) is also verified.

For Lemma (8), it is enough to guarantee that $\sigma_{n}(t)$ is bounded away from zero and above by universal constants that do not depend on $P \in \mathcal{P}_{0}$. This is guaranteed by the assumption that $f_{X}$ and $f_{Y}$ are bounded away from zero and above by universal constants that do not depend on $P \in \mathcal{P}_{0}$.

A.5.3. Proof of (iii). Let $c_{a}^{D}$ be a constant such that $\sqrt{n} h^{\beta-1 / 2} c_{a}^{D}$ is the $(1-a)$-th quantile of $\sup _{t \in \mathcal{T}} \mathcal{G}_{n, m}^{D}(t)$. Using equation (A.10) and mirroring the arguments in the proof of Theorem 1 , we have that

$$
P\left\{\sqrt{n} h^{\beta-1 / 2} \hat{c}_{\alpha}^{D}<\sqrt{n} h^{\beta-1 / 2} c_{\alpha-\delta_{1 n}}^{D}+\epsilon_{1 n}\right\} \geq 1-\delta_{2 n}
$$


Under $H_{1}$, there exists $t^{*} \in \mathcal{T}$ such that $\mu=F_{X^{*}}\left(t^{*}\right)-F_{Y^{*}}\left(t^{*}\right)>0$. Then we obtain

$$
\begin{aligned}
P\left\{D_{n . m}>\hat{c}_{\alpha}^{D}\right\} & \geq P\left\{\sqrt{n} h^{\beta-1 / 2} D_{n, m}>\sqrt{n} h^{\beta-1 / 2} c_{\alpha-\delta_{1 n}}^{D}+\epsilon_{1 n}\right\}-\delta_{2 n} \\
& \geq P\left\{\mathcal{G}_{n, m}^{D}\left(t^{*}\right)>\sqrt{n} h^{\beta-1 / 2} c_{\alpha-\delta_{1 n}}^{D}-\sqrt{n} h^{\beta-\frac{1}{2}} \mu+\epsilon_{1 n}+\epsilon_{n}\right\}-\delta_{2 n}-\delta_{n},
\end{aligned}
$$

for some $\epsilon_{n}, \delta_{n}=O\left(n^{-c^{\prime}}\right)$ with some $c^{\prime}>0$, where the first inequality follows from (A.12) and the second inequality follows from (A.9). By analogous arguments as in the proof of Theorem 5, we can show $\sqrt{n} h^{\beta-1 / 2} c_{\alpha-\delta_{1 n}}^{D}=O(\sqrt{\log (1 / h)})$. However under Assumption OS (iii), $\sqrt{n} h^{\beta-1 / 2} \log ^{-1 / 2}(1 / h) \mu \rightarrow+\infty$; hence the conclusion follows immediately.

\section{Appendix B. Lemmas}

Hereafter we use the following notation. By the Ito isometry, the variance function of the Gaussian process $\mathcal{G}_{n}$ can be shown to be

$$
\sigma_{n}(t)=h r^{2}(h) \int \overline{\mathbb{L}}^{2}(a) f_{X}(t-h a) d a
$$

Let $\bar{\sigma}_{n}=\sup _{t} \sigma_{n}(t)$ and $\underline{\sigma}_{n}=\inf _{t} \sigma_{n}(t)$. Assumption C (i) $\left(\inf _{t \in \mathcal{T}} f_{X}(t)>c>0\right)$ guarantees that $\underline{\sigma}_{n}>0$ for all $n \in \mathbb{N}$.

Also, define the variance sub-metric $d_{n}(s, t)=\operatorname{Var}\left(\mathcal{G}_{n}(s)-\mathcal{G}_{n}(t)\right)$ on $\mathcal{T}$.

\section{B.1. Lemma for Theorem 1.}

\section{Lemma 2.}

(i): Under Assumptions $C$ and $S S$, it holds that for some $c>0$,

$$
\sqrt{n} h^{\beta-1 / 2} \sup _{t \in \mathcal{T}}\left|\tilde{F}_{X^{*}}(t)-\hat{F}_{X^{*}}(t)\right|=o_{p}\left(n^{-c}\right) .
$$

(ii): Under Assumptions $C$ and $S S$, it holds that for some $c>0$,

$$
\frac{\sqrt{n}}{\varsigma(h)} \sup _{t \in \mathcal{T}}\left|\tilde{F}_{X^{*}}(t)-\hat{F}_{X^{*}}(t)\right|=o_{p}\left(n^{-c}\right) .
$$

Proof. Since the proof is similar, we only present the proof for (i). For simplicity, we restrict attention to the case of $N_{i}=2$. For more general situations where $N_{i}$ is arbitrary but bounded above by $C$, the proof follows by similar arguments after accounting for the dependence structure in $\hat{f}_{\epsilon}^{\mathrm{ft}}$.

We first make the following preliminary observations. Note that $\tilde{F}_{X^{*}}(t)$ can be alternatively written as

$$
\tilde{F}_{X^{*}}(t)=\frac{1}{2 \pi} \int_{-1 / h}^{1 / h} \frac{\operatorname{Im}\left\{e^{\mathrm{i} \omega t} \hat{f}_{X}^{\mathrm{ft}}(\omega)\right\}}{-\omega} \frac{K^{\mathrm{ft}}(h \omega)}{\hat{f}_{\epsilon}^{\mathrm{ft}}(\omega)} d \omega
$$


A similar expression holds for $\hat{F}_{X^{*}}$. Recall that $\xi=\left(f_{\epsilon}^{\mathrm{ft}}\right)^{2}$ and $\hat{\xi}=\left(\hat{f}_{\epsilon}^{\mathrm{ft}}\right)^{2}$ are the population and empirical Fourier transforms of $X_{i, j_{1}}-X_{i, j_{2}}$. We note the following properties for $\hat{\xi}$

$$
\begin{aligned}
E\left[\int_{\omega_{0}}^{h^{-1}} \omega^{-a}|\hat{\xi}(\omega)-\xi(\omega)|^{2} d \omega\right] & = \begin{cases}n^{-1} h^{-(1-a)} & \text { if } a<1 \\
n^{-1} & \text { if } a>1 \\
n^{-1} \log (1 / h) & \text { if } a=1\end{cases} \\
\sup _{|\omega| \leq h^{-1}}|\xi / \hat{\xi}| & \leq 1+o_{p}(1) .
\end{aligned}
$$

The results in (B.2) can be shown by expanding the expectations. To show (B.3), we use Yukich (1987, Theorem 6.3) which assures that under Assumption B (i), $\sup _{|\omega| \leq h^{-1}}|\hat{\xi}-\xi|=$ $O_{p}(\sqrt{\log n / n})$ for $h=O\left(n^{-c}\right)$ with some $c>0$. Combined with Assumption B (ii), this implies $\left\{\min _{|\omega| \leq h^{-1}}|\hat{\xi}|\right\}^{-1}=O_{p}\left(h^{-2 \beta}\right)$. Thus we obtain

$$
\sup _{|\omega| \leq h^{-1}}|\xi / \hat{\xi}| \leq 1+\sup _{|\omega| \leq h^{-1}}|(\hat{\xi}-\xi) / \hat{\xi}|=1+O_{p}\left(\left(\frac{\log n}{n h^{4 \beta}}\right)^{1 / 2}\right)=1+o_{p}(1),
$$

thereby proving (B.3).

Pick any $\eta \in(1 / 2, \gamma-\beta)$. Under Assumptions C (iii) and OS (i), it can be verified that

$$
\int_{-1 / h}^{1 / h}\left|\frac{\omega^{\eta} f_{X^{*}}^{\mathrm{ft}}(\omega)}{\xi(\omega)^{1 / 2}}\right|^{2} d \omega=O(1) .
$$

We shall also make frequent use of the following algebraic inequality:

$$
\left|\hat{\xi}^{1 / 2}-\xi^{1 / 2}\right| \leq \xi^{-1 / 2}|\hat{\xi}-\xi|
$$

We now proceed to the main part of the proof. By (B.1), we can expand

$$
\begin{aligned}
\tilde{F}_{X^{*}}(t)-\hat{F}_{X^{*}}(t)= & \frac{1}{\pi} \int_{0}^{\omega_{0}} \frac{\operatorname{Im}\left\{e^{-\mathrm{i} \omega t} \hat{f}_{X}^{\mathrm{ft}}(\omega)\right\}}{-\omega}\left\{\hat{\xi}(\omega)^{-1 / 2}-\xi(\omega)^{-1 / 2}\right\} K^{\mathrm{ft}}(h \omega) d \omega \\
& +\frac{1}{\pi} \int_{\omega_{0}}^{1 / h} \frac{\operatorname{Im}\left\{e^{-\mathrm{i} \omega t} \hat{f}_{X}^{\mathrm{ft}}(\omega)\right\}}{-\omega}\left\{\hat{\xi}(\omega)^{-1 / 2}-\xi(\omega)^{-1 / 2}\right\} K^{\mathrm{ft}}(h \omega) d \omega \\
= & B_{1 n}(t)+B_{2 n}(t) .
\end{aligned}
$$

For the term $B_{1 n}(t)$, using (B.5), we have

$$
\left|B_{1 n}(t)\right| \leq \frac{1}{\pi} \int_{0}^{\omega_{0}}\left|\frac{\operatorname{Im}\left\{e^{-i \omega t} \hat{f}_{X}^{\mathrm{ft}}(\omega)\right\}}{-\omega}\right|\left|\frac{\xi(\omega)}{\hat{\xi}(\omega)}\right|^{1 / 2} \frac{|\hat{\xi}(\omega)-\xi(\omega)|}{\xi(\omega)^{3 / 2}} d \omega .
$$

By the fact $\sup _{|\omega| \leq \omega_{0}}|\hat{\xi}-\xi|=O_{p}\left(n^{-1 / 2}\right)$ and (B.3), we obtain

$$
\sup _{t \in \mathcal{T}}\left|B_{1 n}(t)\right|=O_{p}\left(n^{-1 / 2}\right) \sup _{t \in \mathcal{T}} I(t),
$$


where

$$
\begin{aligned}
I(t) & =\int_{0}^{\omega_{0}}\left|\frac{\operatorname{Im}\left\{e^{-\mathrm{i} \omega t} \hat{f}_{X}^{\mathrm{ft}}(\omega)\right\}}{-\omega}\right| d \omega \\
& \leq \int_{0}^{\omega_{0}}\left|\frac{\sin (\omega t)}{\omega} \operatorname{Re}\left\{\hat{f}_{X}^{\mathrm{ft}}(\omega)\right\}\right| d \omega+\int_{0}^{\omega_{0}}\left|\frac{\cos (\omega t)}{\omega} \operatorname{Im}\left\{\hat{f}_{X}^{\mathrm{ft}}(\omega)\right\}\right| d \omega \\
& \leq \int_{0}^{\omega_{0}}\left|\frac{\sin (\omega t)}{\omega}\right| d \omega+\int_{0}^{\omega_{0}}\left|\frac{\operatorname{Im}\left\{\hat{f}_{X}^{\mathrm{ft}}(\omega)\right\}}{\omega}\right| d \omega \\
& =I_{1}(t)+I_{2} .
\end{aligned}
$$

Since $\mathcal{T}$ is a compact set, it holds $\sup _{t \in \mathcal{T}} I_{1}(t)<\infty$. By the definition of $\hat{f}_{X}^{\mathrm{ft}}$, the random variable $I_{2}$ can be bounded as

$$
I_{2} \leq \frac{1}{N} \sum_{i, j} \int_{0}^{\omega_{0}}\left|\frac{\sin \left(\omega X_{i, j}\right)}{\omega}\right| d \omega \equiv \frac{1}{N} \sum_{i, j} T_{i, j} .
$$

Since

$$
E\left[T_{i, j}\right]=E \int_{0}^{\omega_{0}\left|X_{i, j}\right|}\left|\frac{\sin (t)}{t}\right| d t \leq C_{1}+E\left[\log \left|X_{i, j}\right|\right]<\infty
$$

for some $C_{1}>0$, it holds $I_{2}=O_{p}(1)$. Combining these results, we obtain $\sup _{t \in \mathcal{T}}\left|B_{1 n}(t)\right|=$ $O_{p}\left(n^{-1 / 2}\right)$.

For the term $B_{2 n}(t)$, we further expand

$$
\begin{aligned}
B_{2 n}(t)= & -\frac{1}{\pi} \int_{\omega_{0}}^{1 / h} \frac{\operatorname{Im}\left\{e^{-\mathrm{i} \omega t} f_{X}^{\mathrm{ft}}(\omega)\right\}}{-\omega \xi(\omega)^{1 / 2}}\left\{\hat{\xi}(\omega)^{1 / 2}-\xi(\omega)^{1 / 2}\right\} K^{\mathrm{ft}}(h \omega) \frac{\xi(\omega)^{1 / 2}}{\hat{\xi}(\omega)^{1 / 2}} d \omega \\
& +\frac{1}{\pi} \int_{\omega_{0}}^{1 / h} \frac{\operatorname{Im}\left\{e^{-\mathrm{i} \omega t}\left\{\hat{f}_{X}^{\mathrm{ft}}(\omega)-f_{X}^{\mathrm{ft}}(\omega)\right\}\right\}}{-\omega}\left\{\hat{\xi}(\omega)^{-1 / 2}-\xi(\omega)^{-1 / 2}\right\} K^{\mathrm{ft}}(h \omega) d \omega \\
= & B_{21 n}(t)+B_{22 n}(t) .
\end{aligned}
$$

For the term $B_{21 n}(t)$, we have

$$
\begin{aligned}
\sup _{t \in \mathcal{T}}\left|B_{21 n}(t)\right| & \leq \frac{1}{\pi} \int_{\omega_{0}}^{1 / h}\left|\frac{\omega^{\eta} f_{X^{*}}^{\mathrm{ft}}(\omega)}{\xi(\omega)^{1 / 2}}\right|\left|\frac{\hat{\xi}(\omega)-\xi(\omega)}{\omega^{1+\eta} \xi(\omega)^{1 / 2}}\right|\left|\frac{\xi(\omega)}{\hat{\xi}(\omega)}\right|^{1 / 2} d \omega \\
& \leq C_{2}\left(1+o_{p}(1)\right)\left(\int_{\omega_{0}}^{1 / h}\left|\frac{\omega^{\eta} f_{X^{*}}^{\mathrm{ft}}(\omega)}{\xi(\omega)^{1 / 2}}\right|^{2} d \omega\right)^{1 / 2}\left(\int_{\omega_{0}}^{1 / h} \omega^{2(\beta-\eta-1)}|\hat{\xi}(\omega)-\xi(\omega)|^{2} d \omega\right)^{1 / 2} \\
& =O\left(n^{-1 / 2} h^{(\eta-\beta+1 / 2) \wedge 0}\right),
\end{aligned}
$$

for some $C_{2}>0$, where the first inequality follows from the fact $\left|\operatorname{Im}\left\{e^{-\mathrm{i} \omega t} f_{X}^{\mathrm{ft}}(\omega)\right\}\right| \leq\left|f_{X}^{\mathrm{ft}}(\omega)\right|=$ $\left|f_{X^{*}}^{\mathrm{ft}}(\omega)\right| \xi(\omega)^{1 / 2}$ and (B.5), the second inequality follows from (B.3) and Assumption OS (i), and the equality follows from (B.2) and (B.4). 
Now consider the term $B_{22 n}(t)$. Applying (B.5) and Assumption OS (i), we can write

$$
\begin{aligned}
\sup _{t \in \mathcal{T}}\left|B_{22 n}(t)\right| & \leq \frac{1}{\pi} \int_{\omega_{0}}^{1 / h}\left|\hat{f}_{X}^{\mathrm{ft}}(\omega)-f_{X}^{\mathrm{ft}}(\omega)\right||\hat{\xi}(\omega)-\xi(\omega)||\xi(\omega) / \hat{\xi}(\omega)|^{1 / 2} \frac{1}{\omega \xi(\omega)^{3 / 2}} d \omega \\
& \leq \frac{1}{c^{3} \pi} \int_{\omega_{0}}^{1 / h}\left|\hat{f}_{X}^{\mathrm{ft}}(\omega)-f_{X}^{\mathrm{ft}}(\omega)\right||\hat{\xi}(\omega)-\xi(\omega)||\xi(\omega) / \hat{\xi}(\omega)|^{1 / 2} \omega^{3 \beta-1} d \omega
\end{aligned}
$$

for some $c>0$. As in (B.4), it can be shown after expanding the expectation that

$$
E\left[\int_{\omega_{0}}^{1 / h} \omega^{-a}\left|\hat{f}_{X}^{\mathrm{ft}}(\omega)-f_{X}^{\mathrm{ft}}(\omega)\right|^{2} d \omega\right]=O\left(\left(n h^{1-a}\right)^{-1}\right),
$$

for all $a<1$. Thus, by (B.3) and (B.4), it follows

$$
\begin{aligned}
\sup _{t \in \mathcal{T}}\left|B_{22 n}(t)\right| & \leq \frac{1+o_{p}(1)}{\pi} \int_{\omega_{0}}^{1 / h} \omega^{3 \beta-1}\left|\hat{f}_{X}^{\mathrm{ft}}(\omega)-f_{X}^{\mathrm{ft}}(\omega)\right||\hat{\xi}(\omega)-\xi(\omega)| d \omega \\
& =\frac{1+o_{p}(1)}{\pi}\left(\int_{\omega_{0}}^{1 / h} \omega^{3 \beta-1}\left|\hat{f}_{X}^{\mathrm{ft}}(\omega)-f_{X}^{\mathrm{ft}}(\omega)\right|^{2} d \omega\right)^{1 / 2}\left(\int_{\omega_{0}}^{1 / h} \omega^{3 \beta-1}|\hat{\xi}(\omega)-\xi(\omega)|^{2} d \omega\right)^{1 / 2} \\
& =O\left(\left(n h^{3 \beta}\right)^{-1}\right) .
\end{aligned}
$$

Combining these results, we obtain

$$
\sqrt{n} h^{\beta-1 / 2} \sup _{t \in \mathcal{T}}\left|\tilde{F}_{X^{*}}(t)-\hat{F}_{X^{*}}(t)\right|=O_{p}\left(h^{\eta \wedge(\beta-1 / 2)}+\frac{1}{\sqrt{n} h^{2 \beta+1 / 2}}\right)=o_{p}\left(n^{-c}\right)
$$

for some $c>0$, under Assumptions OS (iii), B (ii) and the condition $\eta>1 / 2$.

B.2. Lemma for Theorem 2. An analog of Lemma 2 is obtained as follows.

\section{Lemma 3.}

(i): Suppose that Assumptions C (i)-(ii) C', OS (i), and OS' hold true, and $m n^{\zeta_{0}-1} h^{2 \beta-2 \gamma-1} \rightarrow$ 0 as $n \rightarrow \infty$ for some $0<\zeta_{0}<1$. Then for some $c>0$,

$$
\sqrt{m} h^{\beta-1 / 2} \sup _{t \in \mathcal{T}}\left|\check{F}_{X^{*}}^{A}(t)-\bar{F}_{X^{*}}^{A}(t)\right|=o_{p}\left(n^{-c}\right) .
$$

(ii): Suppose that Assumptions $C$ (i)-(ii) $C^{\prime}, S S$ (i), and $S S^{\prime}$ hold true, and $m n^{\zeta_{1}-1}(\log n)^{2 r+1}(\log \log n)^{2} \rightarrow 0$ as $n \rightarrow \infty$ for some $0<\zeta_{1}<1$. Then for some $c>0$,

$$
\frac{\sqrt{m}}{\varsigma(h)} \sup _{t \in \mathcal{T}}\left|\check{F}_{X^{*}}^{A}(t)-\bar{F}_{X^{*}}^{A}(t)\right|=o_{p}\left(n^{-c}\right) .
$$

Proof. Proof of (i). We prepare the following lemma.

Lemma 4. Suppose that Assumptions C (i)-(ii), C'(i), OS (i), and $O S^{\prime}$ (i) and (iv) hold true.

Then for any $a \leq 2 \gamma+2$,

$$
E\left[\int_{\omega_{0}}^{1 / h} \omega^{-a}\left|\check{f}_{\epsilon}^{\mathrm{ft}}(\omega)-f_{\epsilon}^{\mathrm{ft}}(\omega)\right|^{2} d \omega\right]=O\left(\frac{1}{n h^{2 \gamma+2-a}}\right) .
$$


Proof. [Proof of Lemma 4] From a similar argument in the proof of Comte and Kappus (2015, Theorem 3.2), we have

$$
\begin{aligned}
& \int_{\omega_{0}}^{1 / h} \omega^{-a} E\left[\left|\mathrm{f}_{\epsilon}^{\mathrm{ft}}(\omega)-f_{\epsilon}^{\mathrm{ft}}(\omega)\right|^{2}\right] d \omega \\
\leq & \frac{C_{0}}{n}\left(\int_{\omega_{0}}^{1 / h} \omega^{-a}\left(\int_{0}^{\omega} \frac{1}{\left|f_{X^{*}}^{\mathrm{ft}}(u)\right|^{2}} d u\right) d \omega\right) \\
& +\frac{C_{0}}{n^{p-1}}\left(\int_{\omega_{0}}^{1 / h} \omega^{-a} \frac{1}{\left|f_{X^{*}}^{\mathrm{ft}}(\omega)\right|^{2}}\left(\int_{0}^{\omega} \frac{1}{\left|f_{X^{*}}^{\mathrm{ft}}(u)\right|^{2}} d u\right)\left(\int_{0}^{\omega} \frac{1}{|\psi(0, u)|^{2}} d u\right)^{p-1} d \omega\right) \\
& +\frac{C_{0}}{n^{2}}\left(\int_{\omega_{0}}^{1 / h} \omega^{-a} \frac{1}{\left|f_{X^{*}}^{\mathrm{ft}}(\omega)\right|^{2}}\left(\int_{0}^{\omega} \frac{1}{|\psi(0, u)|^{2}} d u\right) d \omega\right) \\
& +\frac{C_{0}}{n^{p}}\left(\int_{\omega_{0}}^{1 / h} \omega^{-a} \frac{1}{\left|f_{X^{*}}^{\mathrm{ft}}(\omega)\right|^{4}}\left(\int_{0}^{\omega} \frac{1}{|\psi(0, u)|^{2}} d u\right)^{p} d \omega\right)+\frac{C_{0}}{n^{2}} \int_{\omega_{0}}^{1 / h} \omega^{-a} \frac{1}{\left|f_{X^{*}}^{\mathrm{ft}}(\omega)\right|^{4}} d \omega
\end{aligned}
$$

where $C_{0}>0$ is a constant which does not depend on $n$. Thus, Assumptions OS (i) and OS' (i) and (iv) imply the conclusion.

Let $\check{f}_{X}^{\mathrm{ft}}(\omega)=m^{-1} \sum_{i=1}^{m} 2^{-1} \sum_{j=1}^{2} e^{\mathrm{i} \omega X_{i . j}}$ be the empirical characteristic function. We follow the proof of Lemma 2. Decompose

$$
\begin{aligned}
\check{F}_{X^{*}}^{A}(t)-\bar{F}_{X^{*}}^{A}(t)= & \frac{1}{\pi} \int_{0}^{\omega_{0}} \frac{1}{\omega} \operatorname{Im}\left[e^{-\mathrm{i} \omega t} \check{f}_{X}^{\mathrm{ft}}(\omega)\left\{\check{f}_{\epsilon}^{\mathrm{ft}}(\omega)^{-1}-f_{\epsilon}^{\mathrm{ft}}(\omega)^{-1}\right\}\right] K^{\mathrm{ft}}(h \omega) d \omega \\
& +\frac{1}{\pi} \int_{\omega_{0}}^{h^{-1}} \frac{1}{\omega} \operatorname{Im}\left[e^{-\mathrm{i} \omega t} \check{f}_{X}^{\mathrm{ft}}(\omega)\left\{\check{f}_{\epsilon}^{\mathrm{ft}}(\omega)^{-1}-f_{\epsilon}^{\mathrm{ft}}(\omega)^{-1}\right\}\right] K^{\mathrm{ft}}(h \omega) d \omega \\
= & B_{1 n}^{\prime}(t)+B_{2 n}^{\prime}(t) .
\end{aligned}
$$

For the term $B_{1 n}^{\prime}(t)$, we have

$$
\left|B_{1 n}^{\prime}(t)\right| \leq C_{1} \int_{0}^{\omega_{0}}\left|\frac{1}{\omega} \operatorname{Im}\left[e^{-\mathrm{i} \omega t} \check{f}_{X}^{\mathrm{ft}}(\omega)\right]\right|\left|\frac{f_{\epsilon}^{\mathrm{ft}}(\omega)}{\check{f}_{\epsilon}^{\mathrm{ft}}(\omega)}\right|\left|\frac{f_{\epsilon}^{\mathrm{ft}}(\omega)-\check{f}_{\epsilon}^{\mathrm{ft}}(\omega)}{f_{\epsilon}^{\mathrm{ft}}(\omega)^{2}}\right| d \omega
$$

for some $C_{1}>0$. Note that $\sup _{|\omega| \leq \omega_{0}}\left|\check{f}_{\epsilon}^{\mathrm{ft}}(\omega)-f_{\epsilon}^{\mathrm{ft}}(\omega)\right|=O_{p}\left(n^{-1 / 2}\right)$ by Lemma 1 (i). Also by Lemma 1 (i) and Assumption OS' (v),

$$
\begin{aligned}
\sup _{|\omega| \leq h^{-1}}\left|f_{\epsilon}^{\mathrm{ft}}(\omega) / \check{f}_{\epsilon}^{\mathrm{ft}}(\omega)\right| & \leq 1+\frac{\sup _{|\omega| \leq h^{-1}}\left|\check{f}_{\epsilon}^{\mathrm{ft}}(\omega)-f_{\epsilon}^{\mathrm{ft}}(\omega)\right|}{\inf _{|\omega| \leq h^{-1}}\left\{\left|f_{\epsilon}^{\mathrm{ft}}(\omega)\right|-\left|\check{f}_{\epsilon}^{\mathrm{ft}}(\omega)-f_{\epsilon}^{\mathrm{ft}}(\omega)\right|\right\}}=1+O_{p}\left(\frac{\log h^{-1}}{n^{1 / 2} h^{3 \beta+3 \gamma+1}}\right) \\
& =1+o_{p}(1) .
\end{aligned}
$$

Then we obtain $\sup _{t \in \mathcal{T}}\left|B_{1 n}^{\prime}(t)\right|=O_{p}\left(n^{-1 / 2}\right)$ since $\sup _{t \in \mathcal{T}} I(t)=O(1)$. 
For the term $B_{2 n}^{\prime}(t)$, we further decompose

$$
\begin{aligned}
B_{2 n}^{\prime}(t)= & \frac{1}{\pi} \int_{\omega_{0}}^{h^{-1}} \operatorname{Im}\left[\frac{e^{-\mathrm{i} \omega t} f_{X}^{\mathrm{ft}}(\omega)}{\omega f_{\epsilon}^{\mathrm{ft}}(\omega)^{2}} \frac{f_{\epsilon}^{\mathrm{ft}}(\omega)}{\check{f}_{\epsilon}^{\mathrm{ft}}(\omega)}\left\{f_{\epsilon}^{\mathrm{ft}}(\omega)-\check{f}_{\epsilon}^{\mathrm{ft}}(\omega)\right\}\right] K^{\mathrm{ft}}(h \omega) d \omega \\
& +\frac{1}{\pi} \int_{\omega_{0}}^{h^{-1}} \operatorname{Im}\left[\frac{e^{-\mathrm{i} \omega t}}{\omega}\left\{\check{f}_{X}^{\mathrm{ft}}(\omega)-f_{X}^{\mathrm{ft}}(\omega)\right\}\left\{\check{f}_{\epsilon}^{\mathrm{ft}}(\omega)^{-1}-f_{\epsilon}^{\mathrm{ft}}(\omega)^{-1}\right\}\right] K^{\mathrm{ft}}(h \omega) d \omega \\
\equiv & B_{21 n}^{\prime}(t)+B_{22 n}^{\prime}(t) .
\end{aligned}
$$

For the term $B_{21 n}^{\prime}(t)$, the Cauchy-Schwarz inequality and Lemma 4 yield

$$
\begin{aligned}
\sup _{t \in \mathcal{T}}\left|B_{21 n}^{\prime}(t)\right| & \leq \frac{1}{\pi} \int_{\omega_{0}}^{h^{-1}}\left|\frac{\omega^{\eta} f_{X^{*}}^{\mathrm{ft}}(\omega)}{f_{\epsilon}^{\mathrm{ft}}(\omega)}\right|\left|\omega^{-1-\eta}\left\{f_{\epsilon}^{\mathrm{ft}}(\omega)-\check{f}_{\epsilon}^{\mathrm{ft}}(\omega)\right\}\right|\left|\frac{f_{\epsilon}^{\mathrm{ft}}(\omega)}{f_{\epsilon}^{\mathrm{ft}}(\omega)}\right| d \omega \\
& \leq C\left(1+o_{p}(1)\right)\left(\int_{\omega_{0}}^{h^{-1}}\left|\frac{\omega^{\eta} f_{X^{*}}^{\mathrm{ft}}(\omega)}{f_{\epsilon}^{\mathrm{ft}}(\omega)}\right|^{2} d \omega\right)^{1 / 2}\left(\int_{\omega_{0}}^{h^{-1}}\left|\omega^{-1-\eta}\left\{f_{\epsilon}^{\mathrm{ft}}(\omega)-\check{f}_{\epsilon}^{\mathrm{ft}}(\omega)\right\}\right|^{2} d \omega\right)^{1 / 2} \\
& =O_{p}\left(\frac{1}{n^{1 / 2} h^{\gamma-\eta}}\right) .
\end{aligned}
$$

For the term $B_{22 n}^{\prime}(t)$, applying Assumptions OS (i) and OS' (i), we can write

$$
\begin{aligned}
\sup _{t \in \mathcal{T}}\left|B_{22 n}^{\prime}(t)\right| & \leq \frac{1}{\pi} \int_{\omega_{0}}^{h^{-1}}\left|\check{f}_{X}^{\mathrm{ft}}(\omega)-f_{X}^{\mathrm{ft}}(\omega)\right|\left|f_{\epsilon}^{\mathrm{ft}}(\omega)-\check{f}_{\epsilon}^{\mathrm{ft}}(\omega)\right| \frac{f_{\epsilon}^{\mathrm{ft}}(\omega)}{\check{f}_{\epsilon}^{\mathrm{ft}}(\omega)}|| \frac{1}{\omega f_{\epsilon}^{\mathrm{ft}}(\omega)^{2}} \mid d \omega \\
& \leq C\left(1+o_{p}(1)\right)\left(\int_{\omega_{0}}^{h^{-1}} \omega^{2 \beta-1}\left|\breve{f}_{X}^{\mathrm{ft}}(\omega)-f_{X}^{\mathrm{ft}}(\omega)\right|^{2} d \omega\right)^{1 / 2}\left(\int_{\omega_{0}}^{h^{-1}} \omega^{2 \beta-1}\left|f_{\epsilon}^{\mathrm{ft}}(\omega)-\check{f}_{\epsilon}^{\mathrm{ft}}(\omega)\right|^{2} d \omega\right)^{1 / 2} .
\end{aligned}
$$

It can be shown after expanding the expectation that

$$
E\left[\int_{\omega_{0}}^{1 / h} \omega^{-a}\left|\check{f}_{X}^{\mathrm{ft}}(\omega)-f_{X}^{\mathrm{ft}}(\omega)\right|^{2} d \omega\right]=O\left(\left(m h^{1-a}\right)^{-1}\right),
$$

for all $a<1$. Thus, by Lemma 4 , it follows $\sup _{t \in \mathcal{T}}\left|B_{22 n}^{\prime}(t)\right|=O_{p}\left(\frac{1}{\sqrt{m n} h^{2 \beta+\gamma+1 / 2}}\right)$.

Combining these results, we obtain

$$
\sqrt{m} h^{\beta-1 / 2} \sup _{t \in \mathcal{T}}\left|\check{F}_{X^{*}}^{A}(t)-\bar{F}_{X^{*}}^{A}(t)\right|=O_{p}\left(\frac{\sqrt{m}}{\sqrt{n} h^{\gamma-\beta+1 / 2}}+\frac{1}{\sqrt{n} h^{\gamma+\beta+1}}\right)=o_{p}\left(n^{-c}\right),
$$

for some $c>0$.

Proof of (ii). We use the following lemma.

Lemma 5. Suppose that Assumptions $C$ (i)-(ii), $C^{\prime}(i), S S(i), S S^{\prime}(i)$ and (iii) hold true. Then

$$
\begin{aligned}
& E\left[\int_{\omega_{0}}^{1 / h} \omega^{-2 \lambda_{0}-1} \exp \left(\frac{2 \omega^{\lambda}}{\mu}\right)\left|\check{f}_{\epsilon}^{\mathrm{ft}}(\omega)-f_{\epsilon}^{\mathrm{ft}}(\omega)\right|^{2} d \omega\right]=O\left(n^{-1} h^{\left.1+2 \lambda_{0 x}+2 \lambda_{0}-\left[\left(1-\lambda_{x}\right) \vee 0\right]\right)} \exp \left(\frac{4 h^{-\lambda}}{\mu_{x} \wedge \mu}\right)\right), \\
& E\left[\int_{\omega_{0}}^{1 / h} \omega^{2 \lambda_{0 x}-2 \lambda_{0}-1} \exp \left(\frac{2 \omega^{\lambda}}{\mu}-\frac{2 \omega^{\lambda_{x}}}{\mu_{x}}\right)\left|\check{f}_{\epsilon}^{\mathrm{ft}}(\omega)-f_{\epsilon}^{\mathrm{ft}}(\omega)\right|^{2} d \omega\right]=O\left(n^{-1} h^{\left.1+2 \lambda_{0}-\left[\left(1-\lambda_{x}\right) \vee 0\right]\right)} \exp \left(\frac{2 h^{-\lambda}}{\mu}\right)\right) .
\end{aligned}
$$


Lemma 5 can be shown in the same manner as the proof of Lemma 4 . Since the rest of the proof is similar, we omit the proof of the super smooth case.

\section{B.3. Lemmas for Theorem 3 under Assumption OS (i)-(iii).}

Lemma 6. Under Assumptions $C(i)$-(iv) and $O S$ (i)-(iii), there exist sequences $\epsilon_{n}, \delta_{n}=O\left(n^{-c}\right)$ for some $c>0$ such that

$$
P\left\{\sup _{t \in \mathcal{T}}\left|\sqrt{n} h^{\beta-1 / 2}\left\{\tilde{F}_{X^{*}}(t)-F_{X^{*}}(t)\right\}-\mathcal{G}_{n}(t)\right|>\epsilon_{n}\right\}<\delta_{n} .
$$

Proof. By applying the argument in Hall and Lahiri (2008), the bias of the estimator $\tilde{F}_{X^{*}}$ satisfies $\sup _{t \in \mathcal{T}}\left|E\left[\tilde{F}_{X^{*}}(t)\right]-F_{X^{*}}(t)\right|=O\left(h^{\gamma}\right)$. Thus, Assumption OS (iii) guarantees

$$
\sqrt{n} h^{\beta-1 / 2} \sup _{t \in \mathcal{T}}\left|E\left[\tilde{F}_{X^{*}}(t)\right]-F_{X^{*}}(t)\right|=o\left(n^{-\xi}\right) .
$$

So, the bias term is negligible and it is enough to show that

$$
P\left\{\sup _{t \in \mathcal{T}}\left|\sqrt{n} h^{\beta-1 / 2}\left\{\tilde{F}_{X^{*}}(t)-E\left[\tilde{F}_{X^{*}}(t)\right]\right\}-\mathcal{G}_{n}(t)\right|>\epsilon_{n}\right\}<\delta_{n},
$$

for some $\epsilon_{n}, \delta_{n}=O\left(n^{-c}\right)$ with $c>0$. Let $F_{X, n}^{E D F}$ be the empirical distribution function by $\left\{X_{i}\right\}_{i=1}^{n}, \alpha_{n}(x)=\sqrt{n}\left\{F_{X, n}^{E D F}(x)-F_{X}(x)\right\}$ be the empirical process, and

$$
D_{n}(t)=\sqrt{n} h^{\beta-1 / 2}\left\{\tilde{F}_{X^{*}}(t)-E\left[\tilde{F}_{X^{*}}(t)\right]\right\}=h^{\beta-1 / 2} \int \mathbb{L}\left(\frac{t-a}{h}\right) d \alpha_{n}(a) .
$$

Then (B.6) is rewritten as

$$
P\left\{\sup _{t \in \mathcal{T}}\left|D_{n}(t)-\mathcal{G}_{n}(t)\right|>\epsilon_{n}\right\}<\delta_{n},
$$

for some $\epsilon_{n}, \delta_{n}=O\left(n^{-c}\right)$ with $c>0$.

First, we approximate $D_{n}(t)$ by

$$
D_{n, 0}(t)=h^{\beta-1 / 2} \int \overline{\mathbb{L}}\left(\frac{t-a}{h}\right) d \alpha_{n}(a),
$$

Note that both $D_{n}(t)$ and $D_{n, 0}(t)$ are well defined as Lebesgue-Steltjes integrals. ${ }^{9}$ From integration by parts,

$$
\begin{aligned}
D_{n}(t)= & h^{\beta-3 / 2} \int \mathbb{K}\left(\frac{t-a}{h}\right) \alpha_{n}(a) d a \\
& +h^{\beta-1 / 2} \lim _{a \rightarrow \infty}\left\{\mathbb{L}\left(\frac{t-a}{h}\right) \alpha_{n}(a)\right\}-h^{\beta-1 / 2} \lim _{a \rightarrow-\infty}\left\{\mathbb{L}\left(\frac{t-a}{h}\right) \alpha_{n}(a)\right\} \\
= & h^{\beta-3 / 2} \int \mathbb{K}\left(\frac{t-a}{h}\right) \alpha_{n}(a) d a,
\end{aligned}
$$

\footnotetext{
${ }^{9}$ This is verified as follows. By the definition $\mathbb{L}(u)=\int_{0}^{u} \mathbb{K}(v) d v$ and Assumption OS (ii), we have $\sup _{u}|\mathbb{L}(u)|<\infty$. Also, by $\overline{\mathbb{L}}(u)=\int_{0}^{u} \overline{\mathbb{K}}(v) d v$ (follows from Fubini's theorem) and Assumption OS (ii), we have $\sup _{u}|\overline{\mathbb{L}}(u)|<\infty$. Therefore, bounded variation of the empirical process $\alpha_{n}$ guarantees that both $D_{n}(t)$ and $D_{n, 0}(t)$ are well defined.
} 
for all $n \in \mathbb{N}$, where the second equality follows from the facts $\lim _{a \rightarrow \pm \infty} \alpha_{n}(a)=0$ and $\sup _{u}|\mathbb{L}(u)|<\infty$ for each $h$. Since a similar expression applies for $D_{n, 0}(t)$, there exists $C>0$ such that

$$
D_{n}(t)-D_{n, 0}(t)=h^{\beta-1 / 2} \int\{\mathbb{K}(u)-\overline{\mathbb{K}}(u)\} \alpha_{n}(u-t h) d u \leq C h^{s} \sup _{u}\left|\alpha_{n}(u)\right|,
$$

for all $n$ large enough and $t \in \mathcal{T}$, where the inequality follows from Assumption OS (ii). Now by the strong approximation (Komlós, Major and Tusnády, 1975), there exists a tight Brownian bridge $B(t)=W(t)-t W(1)$ and universal constants $C_{1}, C_{2}>0$ such that

$$
P\left\{\sup _{u}\left|\alpha_{n}(u)\right| \leq \sup _{u}\left|B\left(F_{X}(u)\right)\right|+C_{1} \frac{\log n}{\sqrt{n}}\right\} \geq 1-\frac{C_{2}}{n},
$$

for all $n \in \mathbb{N}$. Combining these results and using the properties of $\sup _{u}\left|B\left(F_{X}(u)\right)\right|$ (in particular, $P\left\{\sup _{u}\left|B\left(F_{X}(u)\right)\right| \geq x\right\} \leq 2 \exp \left(-2 x^{2}\right)$ for $\left.x>0\right)$, there exists $C_{3}>0$ such that

$$
P\left\{\sup _{t \in \mathcal{T}}\left|D_{n}(t)-D_{n, 0}(t)\right|>h^{s / 2}\right\} \leq C_{3} \exp \left(-2 h^{-s}\right)+\frac{C_{2}}{n},
$$

for all $n$ large enough. Note that $h^{s / 2}=O\left(n^{-c_{1}}\right)$ for some $c_{1}>0$ due to Assumption OS (iii) $\left(n^{\nu} h \rightarrow 0\right)$. Thus, it is enough for (B.7) to show that

$$
P\left\{\sup _{t \in \mathcal{T}}\left|D_{n, 0}(t)-\mathcal{G}_{n}(t)\right|>\epsilon_{n}\right\}<\delta_{n}
$$

for some $\epsilon_{n}, \delta_{n}=O\left(n^{-c}\right)$ with $c>0$.

Second, we approximate $D_{n, 0}(t)$ by

$$
D_{n, 1}(t)=h^{\beta-1 / 2} \int \overline{\mathbb{L}}\left(\frac{t-a}{h}\right) d B\left(F_{X}(a)\right) .
$$

Since $\overline{\mathbb{L}} \in L_{2}(\mathbb{R})$, this integral exists for all $t \in \mathbb{R}$. Analogous to the integration by parts formula in (B.8), a similar result applies for $D_{n, 1}(t)$ based on stochastic integration by parts using the facts $\lim _{u \rightarrow \pm \infty} \overline{\mathbb{L}}(u)=0$ and $\sup _{a}\left|B\left(F_{X}(a)\right)\right|<\infty$ almost surely. Thus, we have

$$
\begin{aligned}
D_{n, 0}(t)-D_{n, 1}(t) & =h^{\beta-3 / 2} \int \overline{\mathbb{K}}\left(\frac{t-a}{h}\right)\left\{\alpha_{n}(a)-B\left(F_{X}(a)\right)\right\} d a \\
& \leq h^{\beta-1 / 2} \sup _{a}\left|\alpha_{n}(a)-B\left(F_{X}(a)\right)\right| \int|\overline{\mathbb{K}}(u)| d u
\end{aligned}
$$

for all $n \in \mathbb{N}$, almost surely. Now by Komlós, Major and Tusnády (1975), there exist Brownian bridge $B$ with continuous sample paths and universal constants $C_{4}, C_{5}>0$ such that

$$
P\left\{\sup _{a \in \mathbb{R}}\left|\alpha_{n}(a)-B\left(F_{X}(a)\right)\right|>C_{4} \frac{\log n}{\sqrt{n}}\right\} \leq \frac{C_{5}}{n},
$$


for all $n \in \mathbb{N}$. Combining this with Assumption OS (ii) (eq. (2.9)), there exist $c_{2}, C_{6}>0$ such that

$$
P\left\{\sup _{t \in \mathcal{T}}\left|D_{n, 0}(t)-D_{n, 1}(t)\right|>C_{6} n^{-c_{2}}\right\} \leq \frac{C_{5}}{n},
$$

for all $n$ large enough. Thus, it is enough for (B.7) to show that

$$
P\left\{\sup _{t \in \mathcal{T}}\left|D_{n, 1}(t)-\mathcal{G}_{n}(t)\right|>\epsilon_{n}\right\}<\delta_{n}
$$

for some $\epsilon_{n}, \delta_{n}=O\left(n^{-c}\right)$ with $c>0$.

Third, we approximate $D_{n, 1}(t)$ by

$$
D_{n, 2}(t)=h^{\beta-1 / 2} \int \overline{\mathbb{L}}\left(\frac{t-a}{h}\right) d W\left(F_{X}(a)\right) .
$$

By the definition $B(t)=W(t)-t W(1)$, we have

$$
\left|D_{n, 1}(t)-D_{n, 2}(t)\right| \leq h^{\beta-1 / 2}|W(1)|\left|\int \overline{\mathbb{L}}\left(\frac{t-a}{h}\right) f_{X}(a) d a\right|,
$$

for all $n \in \mathbb{N}$. Therefore, for the rate of $\sup _{t \in \mathcal{T}}\left|D_{n, 1}(t)-D_{n, 2}(t)\right|$, we need to characterize the order of $I_{n 1}(t)=\int \overline{\mathbb{L}}\left(\frac{t-a}{h}\right) f_{X}(a) d a$. By the definition of $\overline{\mathbb{L}}$ and

$$
\int_{-\infty}^{\infty} \sin (\omega(t-a)) f_{X}(a) d a=\frac{1}{2 \mathrm{i}}\left\{e^{\mathrm{i} \omega t} f_{X}^{\mathrm{ft}}(-\omega)-e^{-\mathrm{i} \omega t} f_{X}^{\mathrm{ft}}(\omega)\right\}
$$

an application of Fubini's theorem assures

$$
\begin{aligned}
\left|I_{n 1}(t)\right| & =\left|\frac{1}{2 \mathrm{i} \pi} \int_{\omega_{0}}^{1 / h}\left\{e^{\mathrm{i} \omega t} f_{X}^{\mathrm{ft}}(-\omega)-e^{-\mathrm{i} \omega t} f_{X}^{\mathrm{ft}}(\omega)\right\} \frac{K^{\mathrm{ft}}(h \omega)}{\omega f_{\epsilon}^{\mathrm{ft}}(\omega)} d \omega\right| \\
& \leq \frac{1}{\pi} \int_{\omega_{0}}^{1 / h} \omega^{-1} d \omega=O(\log (1 / h)) .
\end{aligned}
$$

where the inequality follows from $\left|f_{X}^{\mathrm{ft}}\right|=\left|f_{X^{*}}^{\mathrm{ft}}\right|\left|f_{\epsilon}^{\mathrm{ft}}\right| \leq\left|f_{\epsilon}^{\mathrm{ft}}\right|$ and $f_{\epsilon}^{\mathrm{ft}}(\omega)=f_{\epsilon}^{\mathrm{ft}}(-\omega)$. Substituting this bound for $I_{n 1}(t)$ into (B.9), we obtain

$$
P\left\{\sup _{t \in \mathcal{T}}\left|D_{n, 1}(t)-D_{n, 2}(t)\right|>M_{n} h^{\beta-1 / 2} \log (1 / h)\right\}=O\left(n^{-c_{3}}\right),
$$

for some $c_{3}>0$ and sequence $M_{n}=\log n$. By Assumption OS (i) $(\beta>1 / 2)$, it holds $M_{n} h^{\beta-1 / 2} \log (1 / h)=O\left(n^{-c_{4}}\right)$ for some $c_{4}>0$. Therefore, it is enough for (B.7) to show that

$$
P\left\{\sup _{t \in \mathcal{T}}\left|D_{n, 2}(t)-\mathcal{G}_{n}(t)\right|>\epsilon_{n}\right\}<\delta_{n}
$$

for some $\epsilon_{n}, \delta_{n}=O\left(n^{-c}\right)$ with $c>0$. But we can see that the process $D_{n, 2}(t)$ has the same finite dimensional distributions as the process $\mathcal{G}_{n}(t)$. Therefore, this trivially holds true and the conclusion is obtained. 
Lemma 7. Under Assumptions $C$ (i)-(iv) and $O S(i)$-(iii), there exist sequences $\epsilon_{1 n}, \delta_{1 n}, \delta_{2 n}=$ $O\left(n^{-c}\right)$ for some $c>0$ such that with probability greater than $1-\delta_{2 n}$,

$$
P^{\#}\left\{\sup _{t \in \mathcal{T}}\left|\sqrt{n} h^{\beta-1 / 2}\left\{\tilde{F}_{X^{*}}^{\#}(t)-\tilde{F}_{X^{*}}(t)\right\}-\tilde{\mathcal{G}}_{n}(t)\right|>\epsilon_{1 n}\right\}<\delta_{1 n},
$$

where $\tilde{\mathcal{G}}_{n}$ is a tight Gaussian process with the same distribution as $\mathcal{G}_{n}$ under $P^{\#}$.

Proof. The proof is essentially a reformulation of that of Bissantz, Dümbgen, Holzmann and Munk (2007, Theorem 2.1). Let $\alpha_{n}^{\#}(t)=\sqrt{n}\left\{F_{X}^{E D F}, n-F_{X, n}^{E D F}(t)\right\}$ denote the bootstrap empirical process. As shown in the proof of Bissantz, Dümbgen, Holzmann and Munk (2007, eq. (21)), based on Shorack (1982), there exist a Brownian bridge $B_{n}^{\#}$ and universal constants $C, C_{1}>0$ such that for all $n \in \mathbb{N}$,

$$
P^{\#}\left\{\sup _{t \in \mathbb{R}}\left|\alpha_{n}^{\#}(t)-B_{n}^{\#}\left(F_{X, n}^{E D F}(t)\right)\right|>C \frac{\log n}{\sqrt{n}}\right\} \leq \frac{C_{1}}{n},
$$

almost surely. Now it is known that the Brownian bridge is Hölder continuous for every exponent $b \in(0,1 / 2)$ almost surely. Furthermore, by Komlós, Major and Tusnády's (1975) coupling, along with the fact $P\left\{\sup _{t}\left|B\left(F_{X}(t)\right)\right| \geq \log n\right\} \leq 2 \exp \left(-2(\log n)^{2}\right)$, there exist universal constants $C_{2}, C_{3}>0$ such that

$$
P\left\{\sup _{t \in \mathbb{R}}\left|F_{X, n}^{E D F}(t)-F_{X}(t)\right|>C_{2} \frac{\log n}{\sqrt{n}}\right\} \leq \frac{C_{3}}{n},
$$

for all $n \in \mathbb{N}$, which consequently implies

$$
P\left\{\sup _{t \in \mathbb{R}}\left|B_{n}^{\#}\left(F_{X, n}^{E D F}(t)\right)-B_{n}^{\#}\left(F_{X}(t)\right)\right|>C_{4} \frac{\log n}{n^{b / 2}}\right\} \leq \frac{C_{5}}{n},
$$

for some universal constants $C_{4}, C_{5}>0$. Combining these results, there exist universal constants $C_{6}, C_{7}, C_{8}>0$ such that with probability greater than $1-C_{6} / n$, it holds

$$
P^{\#}\left\{\sup _{t \in \mathbb{R}}\left|\alpha_{n}^{\#}(t)-B_{n}^{\#}\left(F_{X}(t)\right)\right|>C_{7} \frac{\log n}{n^{b / 2}}\right\} \leq \frac{C_{8}}{n},
$$

for all $n \in \mathbb{N}$. Based on this, the conclusion follows by similar arguments to the proof of Lemma 6.

Lemma 8. Suppose that Assumptions $C$ (i)-(iv) and OS (i)-(iii) hold true. Then for any sequence $\epsilon_{n}=O\left(n^{-c}\right)$ with $c>0$, there exists a constant $M>0$ such that

$$
p_{\epsilon_{n}}\left(\mathcal{G}_{n}\right) \leq M \epsilon_{n} \sqrt{\log (1 / h)}
$$

for all $n$ large enough. 
Proof. Pick any $\varepsilon>0$. By Chernozhukov, Chetverikov and Kato (2015, Theorem 3) and separability of $\mathcal{G}_{n}$, there exists $C>0$ such that

$$
p_{\varepsilon}\left(\mathcal{G}_{n}\right) \leq C \varepsilon\left\{\underline{\sigma}_{n}^{-1} E\left[\sup _{t \in \mathcal{T}}\left|\mathcal{G}_{n}(t)\right|\right]+\sqrt{1 \vee \log \left(\underline{\sigma}_{n} / \varepsilon\right)}\right\},
$$

for all $n \in \mathbb{N}$. Thus, it is enough to show that

$$
E\left[\sup _{t \in \mathcal{T}}\left|\mathcal{G}_{n}(t)\right|\right]=O(\sqrt{\log (1 / h)}) .
$$

Now,

$$
d_{n}^{2}(s, t)=h^{2 \beta} \int\left\{\overline{\mathbb{L}}\left(\frac{s}{h}-a\right)-\overline{\mathbb{L}}\left(\frac{t}{h}-a\right)\right\}^{2} f_{X}(h a) d a
$$

by the Ito isometry. Note that $\overline{\mathbb{L}}$ is Lipschitz continuous because its derivative $\overline{\mathbb{K}}$ is uniformly bounded on $\mathbb{R}$ (because $h^{\beta} \sup _{u}|\overline{\mathbb{K}}(u)| \leq C$ for some $C>0$ by Assumption OS (i)). Thus, it holds

$$
d_{n}(s, t) \leq C_{1} h^{-3 / 2}|s-t|
$$

for some $C_{1}>0$ that is independent of $s$ and $t$.

Let $D\left(\varepsilon, d_{n}\right)$ be the $\varepsilon$-packing number for the set $\mathcal{T}$ under the sub-metric $d_{n}$. By (B.10), it holds $D\left(\varepsilon, d_{n}\right) \leq 2 C_{1} h^{-3 / 2} / \varepsilon$. Pick any $\delta \in(0,1)$. By van der Vaart and Wellner (1996, Corollary 2.2.8), there exist universal constants $C_{2}, C_{3}>0$ such that

$$
\begin{aligned}
& E\left[\sup _{d_{n}(s, t) \leq \delta}\left|\mathcal{G}_{n}(s)-\mathcal{G}_{n}(t)\right|\right] \\
\leq & C_{2} \int_{0}^{\delta} \sqrt{\log D\left(\varepsilon, d_{n}\right)} d \varepsilon \leq C_{2} \delta \sqrt{\log \left(2 C_{1} h^{-3 / 2}\right)}+C_{2} \int_{0}^{\delta} \sqrt{\log (1 / \varepsilon)} d \varepsilon \leq C_{3} \sqrt{\log (1 / h)},
\end{aligned}
$$

for all $n \in \mathbb{N}$. Thus, by the above and (B.10), there exists a collection of Gaussian random variables $\left\{\mathcal{G}_{n}\left(t_{j}\right)\right\}_{i=1}^{p_{n}}$ with $p_{n}=\left\lceil\frac{1}{h^{3 / 2} \delta}\right\rceil$ such that

$$
E\left[\sup _{t \in \mathcal{T}}\left|\mathcal{G}_{n}(t)\right|\right] \leq E\left[\max _{1 \leq j \leq p_{n}}\left|\mathcal{G}_{n}\left(t_{j}\right)\right|\right]+C_{3} \sqrt{\log (1 / h)}
$$

for all $n \in \mathbb{N}$. Now the properties of the maximum of Gaussian random variables yields

$$
E\left[\max _{1 \leq j \leq p_{n}}\left|\mathcal{G}_{n}\left(t_{j}\right)\right|\right] \leq 2 \bar{\sigma}_{n} \sqrt{1+\log p_{n}} .
$$

Combining these results, the conclusion follows.

\section{B.4. Lemmas for Theorem 3 under Assumption SS (i)-(iii).}


Lemma 9. Under Assumptions $C(i)$-(iv) and $S S$ (i)-(iii), there exist sequences $\epsilon_{n}=O(\log n)^{-(1+c)}$ and $\delta_{n}=O\left(n^{-c}\right)$ with $c>0$ such that

$$
P\left\{\sup _{t \in \mathcal{T}}\left|\frac{\sqrt{n}}{\varsigma(h)}\left\{\tilde{F}_{X^{*}}(t)-F_{X^{*}}(t)\right\}-\mathcal{G}_{n}(t)\right|>\epsilon_{n}\right\}<\delta_{n} .
$$

Lemma 10. Under Assumptions $C$ (i)-(iv) and $S S$ (i)-(iii), there exist sequences $\epsilon_{1 n}, \delta_{1 n}, \delta_{2 n}=$ $O\left(n^{-c}\right)$ with $c>0$ such that with probability greater than $1-\delta_{2 n}$,

$$
P^{\#}\left\{\sup _{t \in \mathcal{T}}\left|\frac{\sqrt{n}}{\varsigma(h)}\left\{\tilde{F}_{X}^{\#}(t)-\tilde{F}_{X}(t)\right\}-\tilde{\mathcal{G}}_{n}(t)\right|>\epsilon_{1 n}\right\}<\delta_{1 n},
$$

where $\tilde{\mathcal{G}}_{n}$ is a tight Gaussian process with the same distributions as $\mathcal{G}_{n}$ under $P^{\#}$.

These lemmas can be shown in the same way as Lemmas 6 and 7. The log rate of $\epsilon_{n}$ in Lemma 9 is due to the bias term. Recall that under Assumption C (ii), the bias of the estimator $\tilde{F}_{X^{*}}$ is given by

$$
\sup _{t \in \mathcal{T}}\left|E\left[\tilde{F}_{X^{*}}(t)\right]-F_{X^{*}}(t)\right|=O\left(h^{\gamma}\right) .
$$

Then due to Assumption SS (iii), it holds $\sqrt{n} h^{\gamma} / \varsigma(h)=C(\log n)^{-c}$ for some $c>1$.

Lemma 11. Suppose that Assumptions $C$ (i)-(iv) and SS (i)-(iii) hold true. Then for any sequence $\epsilon_{n}=O(\log n)^{-c}$ with $c>1$ there exists $M>0$ such that

$$
p_{\epsilon_{n}}\left(\mathcal{G}_{n}\right) \leq M \epsilon_{n}(\log n)^{1+r}
$$

for all $n$ large enough and any $r>0$ independent of $n$.

Proof. Pick any $\varepsilon>0$. By Chernozhukov, Chetverikov and Kato (2015, Theorem 3) and separability of the Gaussian process $\mathcal{G}_{n}$, there exists $C>0$ such that

$$
p_{\varepsilon}\left(\mathcal{G}_{n}\right) \leq C \varepsilon\left\{\underline{\sigma}_{n}^{-1} E\left[\sup _{t \in \mathcal{T}}\left|\mathcal{G}_{n}(t)\right|\right]+\sqrt{1 \vee \log \left(\underline{\sigma}_{n} / \varepsilon\right)}\right\},
$$

for all $n \in \mathbb{N}$. By Lemmas 12 and 13 shown below, the following hold true:

there exist $c_{1}>0$ such that $\underline{\sigma}_{n} \geq c_{1} h^{\lambda+\nu}$ for all $\nu>0$ and $n$ large enough,

$$
\text { there exist } C_{1}>0 \text { such that } \bar{\sigma}_{n} \leq C_{1} \text { for all } n \text { large enough. }
$$

Observe that

$$
d_{n}^{2}(s, t)=\frac{h}{\varsigma^{2}(h)} \int\left\{\overline{\mathbb{L}}\left(\frac{s}{h}-a\right)-\overline{\mathbb{L}}\left(\frac{t}{h}-a\right)\right\}^{2} f_{X}(h a) d a
$$

by the Ito isometry. Note that $\overline{\mathbb{L}}$ is Lipschitz continuous because its derivative $\overline{\mathbb{K}}$ is uniformly bounded on $\mathbb{R}$ (because $\sqrt{h} \varsigma^{-1}(h) \sup _{u}|\overline{\mathbb{K}}(u)| \leq C_{2} h^{-c_{2}}$ for some $C_{2}, c_{2}>0$ by Assumption SS (i)). Thus, it holds $d_{n}(s, t) \leq C_{3} h^{-c_{2}-3 / 2}|s-t|$ for some $C_{3}>0$ that is independent 
of $s$ and $t$. Using (B.12), an analogous argument as in the proof of Lemma 8 shows that $E\left[\sup _{t \in \mathcal{T}}\left|\mathcal{G}_{n}(t)\right|\right]=O(\sqrt{\log (1 / h)})$. Combining this with (B.11) and Assumption SS (iii), the conclusion follows.

Lemma 12. Under Assumptions $C(i)$-(iv) and $S S$ (i)-(iii), there exists $c>0$ such that $\underline{\sigma}_{n} \geq$ $c h^{\lambda+\nu}$ for all $\nu>0$ and $n$ large enough.

Proof. We only prove the case of $\lambda_{0} \geq 0$. The proof for the case of $\lambda_{0}<0$ is similar. Pick any $\varepsilon>0$. By Assumption C (i), we provide a lower bound for $\underline{\sigma}_{n}$ via

$$
\underline{\sigma}_{n}=\inf _{t \in \mathcal{T}} \frac{h}{\varsigma^{2}(h)} \int \overline{\mathbb{L}}^{2}(a) f_{X}(t-h a) d a \geq \frac{c_{1} h}{\varsigma^{2}(h)} \int_{|a| \leq h^{\varepsilon}} \overline{\mathbb{L}}^{2}(a) d a,
$$

for some $c_{1}>0$. Let

$$
\Phi_{\epsilon}(\omega)=f_{\epsilon}^{\mathrm{ft}}(\omega)^{-1} \mathbb{I}\left\{|\omega| \geq \omega_{0}\right\} .
$$

Using the fact $\sin (x)=x+R(x)$ with $|R(x)| \leq c_{2}|x|^{2}$ for some $c_{2}>0$, it follows that for all $|a| \leq h^{\varepsilon}$,

$$
|\overline{\mathbb{L}}(a)| \geq \frac{1}{\pi}\left|a \int_{0}^{1} K^{\mathrm{ft}}(\omega) \Phi_{\epsilon}\left(\frac{\omega}{h}\right) d \omega\right|-\frac{c_{2}}{\pi}\left|a \int_{0}^{1}\right| a \omega\left|K^{\mathrm{ft}}(\omega) \Phi_{\epsilon}\left(\frac{\omega}{h}\right) d \omega\right| \geq C\left\{1-O\left(h^{\varepsilon}\right)\right\}\left|a I_{n}\right|,
$$

where $I_{n}=\int_{0}^{1} K^{\mathrm{ft}}(\omega) \Phi_{\epsilon}\left(\frac{\omega}{h}\right) d \omega$ and the last inequality follows from the fact $\sup \{|a \omega|:|a| \leq$ $\left.h^{\varepsilon}, \omega \in[0,1]\right\}=h^{\varepsilon}$.

We now provide a lower bound for $I_{n}$. Pick any $\delta>0$. Observe that

$$
\begin{aligned}
h^{\frac{1-\lambda}{2}} \varsigma(h)^{-\frac{1}{2}}\left|I_{n}\right| & =\frac{\exp \left(-1 / \mu h^{\lambda}\right)}{h^{\lambda(r+1)+\lambda_{0}}} \int_{h \omega_{0}}^{1} K^{\mathrm{ft}}(\omega) \Phi_{\epsilon}\left(\frac{\omega}{h}\right) d \omega \\
& \geq c_{3} \frac{\exp \left(-1 / \mu h^{\lambda}\right)}{h^{\lambda(r+1)}} \int_{h \omega_{0}}^{1} K^{\mathrm{ft}}(\omega) \omega^{-\lambda_{0}} \exp \left(\frac{|\omega|^{\lambda}}{h^{\lambda} \mu}\right) d \omega \\
& \geq c_{3} \frac{\exp \left(-1 / \mu h^{\lambda}\right)}{h^{\lambda(r+1)}} \int_{\delta}^{1} K^{\mathrm{ft}}(\omega) \exp \left(\frac{|\omega|^{\lambda}}{h^{\lambda} \mu}\right) d \omega \\
& =c_{3} \int_{0}^{(1-\delta) h^{-\lambda}} \frac{K^{\mathrm{ft}}\left(1-h^{\lambda} v\right)}{\left(h^{\lambda} v\right)^{r}} v^{r} \exp \left(\frac{\left|1-h^{\lambda} v\right|^{\lambda}-1}{h^{\lambda} \mu}\right) d v \\
& \rightarrow c_{3} q^{r} \int v^{r} \exp (-\lambda v / \mu) d v>0
\end{aligned}
$$

for some $c_{3}>0$, where the first inequality follows from the fact $\Phi_{\epsilon}(\omega) \geq c_{3}|\omega|^{-\lambda_{0}} \exp \left(|\omega|^{\lambda} / \mu\right)$, the second inequality holds since all the terms inside the integral are positive and $\omega^{-\lambda_{0}} \mathbb{I}\left\{h \omega_{0} \leq \omega \leq\right.$ $1\} \geq 1$ for $\lambda_{0} \geq 0$, the second equality follows from a change of variables, and the convergence 
follows from the dominated convergence theorem after noting

$$
\begin{aligned}
& \frac{K^{\mathrm{ft}}\left(1-h^{\lambda} v\right)}{\left(h^{\lambda} v\right)^{r}} v^{r} \exp \left(\frac{\left|1-h^{\lambda} v\right|^{\lambda}-1}{h^{\lambda} \mu}\right) \mathbb{I}\left\{0 \leq v \leq(1-\delta) h^{-\lambda}\right\} \\
\leq & \begin{cases}\sup _{0 \leq t \leq 1}\left\{t^{-r} K^{\mathrm{ft}}(1-t)\right\} v^{r} \exp (-v / \mu) & \text { if } \lambda \geq 1, \\
\sup _{0 \leq t \leq 1}\left\{t^{-r} K^{\mathrm{ft}}(1-t)\right\} v^{r} \exp (-\lambda v / \mu) & \text { if } 0<\lambda<1 .\end{cases}
\end{aligned}
$$

Thus, it holds $h^{1 / 2} \varsigma(h)^{-1 / 2}\left|I_{n}\right|>c_{3} h^{\lambda / 2}$ for all $n$ large enough.

Combining these results, there exists $c>0$ such that

$$
\underline{\sigma}_{n} \geq c h^{\lambda} \int_{|a| \leq h^{\varepsilon}}|a|^{2} d a \geq c h^{\lambda+3 \varepsilon},
$$

for all $n$ large enough, and the conclusion follows.

Lemma 13. Under Assumptions $C\left(\right.$ (i)-(iv) and $S S$ (i)-(iii), there exists $C>0$ such that $\bar{\sigma}_{n} \leq C$ for all $n$ large enough.

Proof. We only prove the case of $\lambda_{0} \geq 0$. The proof for the case of $\lambda_{0}<0$ is similar. Pick any $\varepsilon \in\left(0,2^{-1 / \lambda}\right)$. Since $f_{X}$ is bounded (Assumption C (ii)), there exists $C_{1}, C_{2}>0$ such that

$$
\begin{aligned}
\bar{\sigma}_{n} \leq & C_{1} \frac{\exp \left(-2 / \mu h^{\lambda}\right)}{h^{\lambda(2 r+1)+2 \lambda_{0}}} \int \overline{\mathbb{L}}^{2}(a) d a=C_{2} \frac{\exp \left(-2 / \mu h^{\lambda}\right)}{h^{\lambda(2 r+1)+2 \lambda_{0}}} \int_{h \omega_{0}}^{1}\left|\frac{K^{\mathrm{ft}}(\omega)}{\omega} \Phi_{\epsilon}\left(\frac{\omega}{h}\right)\right|^{2} d \omega \\
\leq & C_{2} \omega_{0}^{-2} \frac{\exp \left(-2 / \mu h^{\lambda}\right)}{h^{\lambda(2 r+1)+2\left(1+\lambda_{0}\right)}} \int_{h \omega_{0}}^{1}\left|K^{\mathrm{ft}}(\omega) \Phi_{\epsilon}\left(\frac{\omega}{h}\right)\right|^{2} d \omega \\
\leq & C_{2} \omega_{0}^{-2} \frac{\exp \left(-2 / \mu h^{\lambda}\right)}{h^{\lambda(2 r+1)+2\left(1+\lambda_{0}\right)}} \int_{h \omega_{0}}^{\varepsilon}\left|K^{\mathrm{ft}}(\omega)\left(\frac{\omega}{h}\right)^{-\left(1+\lambda_{0}\right)} \exp \left(\frac{|\omega|^{\lambda}}{h^{\lambda} \mu}\right)\right|^{2} d \omega \\
& +C_{2} \omega_{0}^{-2} \frac{\exp \left(-2 / \mu h^{\lambda}\right)}{h^{\lambda(2 r+1)}} \int_{|\omega|>\varepsilon}\left|K^{\mathrm{ft}}(\omega) \omega^{-\left(1+\lambda_{0}\right)} \exp \left(\frac{|\omega|^{\lambda}}{h^{\lambda} \mu}\right)\right|^{2} d \omega \\
= & T_{1 n}+T_{2 n},
\end{aligned}
$$

for all $n$ large enough, where the first equality follows from Plancherel's isometry, ${ }^{10}$ and the second inequality follows from $\Phi_{\epsilon}(\omega) \leq C|\omega|^{-\lambda_{0}} \exp \left(|\omega|^{\lambda} / \mu\right)$. For $T_{1 n}$, Assumption SS (iii) and the restriction $\varepsilon \in\left(0,2^{-1 / \lambda}\right)$ guarantee

$$
\begin{aligned}
T_{1 n} & \leq C_{3} \omega_{0}^{-\left(1+\lambda_{0}\right)} \frac{\exp \left(-2 / \mu h^{\lambda}\right)}{h^{\lambda(2 r+1)+2\left(1+\lambda_{0}\right)}} \int_{h \omega_{0}}^{\varepsilon}\left|K^{\mathrm{ft}}(\omega) \exp \left(\frac{|\omega|^{\lambda}}{h^{\lambda} \mu}\right)\right|^{2} d \omega \\
& \leq C_{4} \frac{\exp \left(-1 / \mu h^{\lambda}\right)}{h^{\lambda(2 r+1)+2\left(1+\lambda_{0}\right)}}=O\left(n^{-c_{1}}\right),
\end{aligned}
$$

\footnotetext{
${ }^{10}$ Note that $\overline{\mathbb{L}}$ is written as $\overline{\mathbb{L}}(u)=\frac{1}{2 \pi} \int_{-1}^{1} \frac{e^{-\mathrm{i} \omega u}}{\omega} \frac{K^{\mathrm{ft}}(\omega)}{f_{\epsilon}^{\mathrm{ft}}(\omega / h)} \mathbb{I}\left\{|\omega| \geq \omega_{0}\right\} d \omega$. This integral exists due to the truncation.
} 
for some $C_{3}, C_{4}, c_{1}>0$. For $T_{2 n}$, note that

$$
T_{2 n} \leq C_{5} \varepsilon^{-\left(1+\lambda_{0}\right)} \frac{\exp \left(-2 / \mu h^{\lambda}\right)}{h^{\lambda(2 r+1)}} \int_{|\omega|>\varepsilon}\left|K^{\mathrm{ft}}(\omega) \exp \left(\frac{|\omega|^{\lambda}}{h^{\lambda} \mu}\right)\right|^{2} d \omega,
$$

for some $C_{5}>0$. By an analogous dominated convergence argument used in the proof of Lemma 12, we can show $T_{2 n}$ converges to some finite constant. Combining these results, the conclusion follows.

\section{Appendix C. Assumptions and proof for Theorem 4}

In this appendix we prove Theorem 4, the asymptotic distribution of $s_{n}$ in (4.3). Basic steps of our proof follow the recipe laid down by Bissantz, Dümbgen, Holzmann and Munk (2007). Importantly, we impose tail conditions on $f_{\epsilon}^{\mathrm{ft}}$ of the form $f_{\epsilon}^{\mathrm{ft}}(\omega)|\omega|^{\beta} \rightarrow C_{\epsilon}$ as $|\omega| \rightarrow \infty$. Based on this, we define

$$
\begin{aligned}
\mathcal{K}(u) & =\frac{1}{2 \pi C_{\epsilon}} \int_{0}^{\infty} e^{-\mathrm{i} \omega u} \omega^{\beta} K^{\mathrm{ft}}(\omega) d \omega+\frac{1}{2 \pi C_{\epsilon}} \int_{-\infty}^{0} e^{-\mathrm{i} \omega u}|\omega|^{\beta} K^{\mathrm{ft}}(\omega) d \omega, \\
\mathcal{L}(u) & =\frac{1}{2 \pi C_{\epsilon}} \int_{0}^{\infty} \sin (\omega u) \omega^{\beta-1} K^{\mathrm{ft}}(\omega) d \omega+\frac{1}{2 \pi C_{\epsilon}} \int_{-\infty}^{0} \sin (\omega u)|\omega|^{\beta} \omega^{-1} K^{\mathrm{ft}}(\omega) d \omega .
\end{aligned}
$$

These are the pointwise limits of $h^{\beta} \mathbb{K}(u)$ and $h^{\beta} \mathbb{L}(u)$ as $h \rightarrow 0$ under some assumptions on $f_{\epsilon}^{\mathrm{ft}}$. In addition to Assumptions OS (i)-(iii), we impose the following conditions.

\section{Assumption G.}

(i): $f_{\epsilon}^{\mathrm{ft}}(\omega)|\omega|^{\beta} \rightarrow C_{\epsilon}$ as $|\omega| \rightarrow \infty$ for some $\beta>1 / 2$.

(ii): $h^{\beta} \int|\mathbb{K}(u)| d u<M$ for some $M>0$ independent of $h . \int|u|^{3 / 2} \sqrt{\log \left(\log ^{+}|u|\right)}|\mathcal{K}(u)| d u<$ $\infty$. For some $\delta>0, \int\left|h^{\beta} \overline{\mathbb{K}}(u)-\mathcal{K}(u)\right| d u=O\left(h^{1 / 2+\delta}\right)$.

(iii): $\lim _{u \rightarrow \pm \infty} \mid \mathcal{L}(u) \sqrt{|u| \log \left(\log ^{+}|u|\right) \mid}=0$. For some $\delta_{1} \in(0,1), \int|\mathcal{L}(u)|^{2-\delta_{1}} d u<\infty$. For some $\delta>0, \sup _{u}\left|h^{\beta} \overline{\mathbb{L}}(u / h)-\mathcal{L}(u / h)\right|=O\left(h^{1 / 2+\delta}\right)$.

(iv): $f_{X}$ and its derivative $f_{X}^{\prime}$ are bounded and continuous on $\mathbb{R}$ such that $\lim _{x \rightarrow \pm \infty}\left|x f_{X}(x) \log \left(\log ^{+}|x|\right)\right|=0$. Also, $\sup _{x}\left|f_{X}^{\prime}(x) f_{X}(x)^{-1 / 2} \sqrt{|x| \log \left(\log ^{+}|x|\right)}\right|<\infty$. Furthermore it holds

$$
\int\left|f_{X}^{\prime}(x) f_{X}(x)^{-1 / 2} \sqrt{|x| \log \left(\log ^{+}|x|\right)}\right| d x<\infty .
$$

These conditions are generalizations and simplifications of the ones in Bissantz, Dümbgen, Holzmann and Munk (2007). Assumption G (i) is stronger than the usual assumption $f_{\epsilon}^{\mathrm{ft}}(\omega)|\omega|^{\beta}<C_{\epsilon}$ as $|\omega| \rightarrow \infty$ but is required for explicit derivation of the limiting distribution.

Assumption G (ii) contains conditions for the deconvolution kernel $\mathbb{K}$. The first condition ensures that $\mathbb{K}$ is $L_{1}$-integrable. A sufficient condition for this is that $1 / f_{\epsilon}^{\mathrm{ft}}(\omega)$ is a polynomial function in $\omega$. Indeed in this case it can be shown from the properties of the Fourier transform 
that $|\mathbb{K}(u)| \sim|u|^{-q}$ as $|u| \rightarrow \infty$ under some conditions on $f_{\epsilon}^{\mathrm{ft}}$. For instance, the choice $r>2$ for $K$ assures $|\mathbb{K}(u)| \sim|u|^{-2}$ under the assumption

$$
\int\left|\left\{\frac{K^{\mathrm{ft}}(\omega)}{f_{\epsilon}^{\mathrm{ft}}(\omega / h)}\right\}^{\prime \prime}\right| d \omega=O\left(h^{-\beta}\right)
$$

A similar condition is given in, for example, Bissantz, Dümbgen, Holzmann and Munk (2007, eq. (13)). $\mathcal{K}$ in (C.1) is the limit of $\overline{\mathbb{K}}$ as $h \rightarrow \infty$ obtained by Assumption G (i). Recall that by Assumption OS (ii), $h^{\beta-\frac{1}{2}} \int|\mathbb{K}(u)-\overline{\mathbb{K}}(u)| d u=O\left(h^{s}\right)$. Additionally, it can be shown from the previous assumptions and properties of the Fourier transform of $\omega^{\beta} K^{\mathrm{ft}}(\omega)$ that $\int \mid h^{\beta} \mathbb{K}(u)-$ $\mathcal{K}(u) \mid d u<\infty$. To obtain the rate $h^{1 / 2+\delta}$ for the latter, we need some additional conditions on the decay of $f_{\epsilon}^{\mathrm{ft}}$. Denote $R(\omega)=f_{\epsilon}^{\mathrm{ft}}(\omega) \omega^{\beta}-C_{\epsilon}$. Then a sufficient condition for the third condition in Assumption G (ii) is that $R(\omega) \sim \omega^{-1 / 2-\delta}$ as $|\omega| \rightarrow \infty$.

Assumption G (iii) contains conditions on the integrated kernel function $\mathbb{L}$. On the first two conditions in Assumption G (iii), we can in fact show the stronger statement that for all the commonly used kernel functions, $\mathcal{L}(u) \sim|u|^{-\beta \wedge 1}$ as $u \rightarrow \pm \infty$. Regarding the third condition in Assumption G (iii), note that we can expand

$$
h^{\beta} \overline{\mathbb{L}}\left(\frac{u}{h}\right)-L\left(\frac{u}{h}\right)=\frac{h^{\beta}}{\pi C_{\epsilon}} \int_{\omega_{1}}^{1 / h} \frac{\sin (\omega u)}{\omega} K^{\mathrm{ft}}(h \omega) \frac{R(\omega)}{\psi^{\mathrm{ft}}(\omega)} d \omega-\frac{h^{\beta}}{\pi C_{\epsilon}} \int_{0}^{\omega_{1}} \sin (\omega u) \omega^{\beta-1} K^{\mathrm{ft}}(h \omega) d \omega .
$$

Standard arguments show that this is of the order $h^{1 / 2+\delta}$ under the assumption $R(\omega) \sim \omega^{-1 / 2-\delta}$ as $|\omega| \rightarrow \infty$.

Assumption G (iv) provides conditions on the decay rates of the pdf $f_{X}$ and its derivative $f_{X}^{\prime}$. Similar assumptions are adopted in the literature (e.g., Bickel and Rosenblatt, 1973).

Based on these conditions, we obtain Theorem 4 with

$$
B=\int \mathcal{L}(a)^{2} d a, \quad b_{n}=(-2 \log h)^{1 / 2}+(-2 \log h)^{-1 / 2} \log \left(\frac{\int\left\{\mathcal{L}^{\prime}(a)\right\}^{2} d a}{4 \pi B}\right)
$$

Furthermore, if we consider the simple hypothesis

$$
H_{0}: F_{X^{*}}(t)=F_{0}(t) \quad \text { for } t \in \mathcal{T}
$$

for some $F_{0}$, a test statistic for $H_{0}$ is $t_{n}^{0}=\sup _{t \in \mathcal{T}}\left|f_{X}(t)^{-1 / 2}\left\{\hat{F}_{X^{*}}(t)-F_{0}(t)\right\}\right|$. Consider the sequence of local alternatives

$$
H_{1 n}: F_{X^{*}}(t)=F_{0}(t)+\gamma_{n} \eta(t) \quad \text { for } t \in \mathcal{T}
$$

where $\eta(t)$ is a continuous function and $\gamma_{n}=\sqrt{n} h^{\beta-1 / 2}(2 \log (1 / h))^{1 / 2}$. By an analogous argument, we can obtain 


$$
P\left\{(-2 \log h)^{1 / 2}\left(B^{-1 / 2} t_{n}^{0}-b_{n}\right) \leq c\right\} \rightarrow \exp (-s(\eta) \exp (-c)),
$$

for all $c \in \mathbb{R}$, where $s(\eta)=\int_{0}^{1} \exp \left(\left(B f_{X^{*}}(a)\right)^{-1 / 2} \eta(a)\right)+\exp \left(-\left(B f_{X^{*}}(a)\right)^{-1 / 2} \eta(a)\right) d a$.

C.1. Proof of Theorem 4. We show that

$$
\sup _{t \in \mathcal{T}}\left|\sqrt{n h^{2 \beta-1} f_{X}(t)^{-1}}\left\{\tilde{F}_{X^{*}}(t)-F_{X^{*}}(t)\right\}-\mathcal{Y}_{n}(t)\right|=o_{p}\left((-\log (h))^{-1 / 2}\right),
$$

where $\mathcal{Y}_{n}=h^{-1 / 2} \int \mathcal{L}\left(\frac{t-a}{h}\right) d W(a)$ is a Gaussian process. Once we obtain (C.3), the conclusion follows by applying the arguments of Bickel and Rosenblatt (1973, Theorem A1). The rate $o_{p}\left((-\log (h))^{-1 / 2}\right)$ is required because later we scale by $(-\log (h))^{1 / 2}$ to obtain the limiting distribution as in Bickel and Rosenblatt (1973).

First, as in the proof of Lemma 6 , the bias term in $Q_{n}(t)$ is negligible and we can restrict attention to the mean zero process

$$
D_{n}(t)=Q_{n}(t)-E\left[Q_{n}(t)\right]=h^{\beta-1 / 2} \int \mathbb{L}\left(\frac{t-a}{h}\right) d \alpha_{n}(a),
$$

where $\alpha_{n}(a)=\sqrt{n}\left\{F_{X, n}^{E D F}(a)-F_{X}(a)\right\}$ is the empirical process, and $F_{X, n}^{E D F}$ is the empirical distribution function by $\left\{X_{i}\right\}_{i=1}^{n}$. We approximate $D_{n}(t)$ by

$$
D_{n, 1}(t)=h^{\beta-1 / 2} \int \overline{\mathbb{L}}\left(\frac{t-a}{h}\right) d W\left(F_{X}(a)\right) .
$$

Indeed the arguments in the proof of Lemma 6 allow us to show

$$
\left.\sup _{t \in \mathcal{T}}\left|D_{n}(t)-D_{n, 1}(t)\right|=O_{p}\left((n h)^{-1 / 2} \log n\right)\right) .
$$

Also, $D_{n, 1}(t)$ has the same finite dimensional distribution as

$$
D_{n, 2}(t)=h^{\beta-1 / 2} \int \overline{\mathbb{L}}\left(\frac{t-a}{h}\right) f_{X}(a)^{1 / 2} d W(a) .
$$

Next, we approximate $D_{n, 2}(t)$ by

$$
D_{n, 3}(t)=h^{-3 / 2} \int \mathcal{K}\left(\frac{t-a}{h}\right) f_{X}(a)^{1 / 2} W(a) d a .
$$

To this end, note that for any $h>0$,

$$
\lim _{a \rightarrow \pm \infty} \mathcal{K}\left(\frac{t-a}{h}\right) f_{X}(a)^{1 / 2} W(a) \leq \sup _{u}|\mathcal{K}(u)| \lim _{a \rightarrow \pm \infty}\left|a f_{X}(a) \log \left(\log ^{+}|a|\right)\right|^{1 / 2}=0,
$$

where the inequality follows from the law of the iterated logarithm for the Wiener process and the equality follows from the facts $\sup _{u}|\mathcal{K}(u)|=O\left(h^{-\beta-1}\right)$ and Assumption G (iv). Thus, using 
stochastic integration by parts, we can write

$$
D_{n, 2}(t)=h^{\beta-1 / 2} \int\left\{f_{X}(t-h u)^{1 / 2} \overline{\mathbb{K}}(u)+h f_{X}^{\prime}(t-h u) f_{X}(t-h u)^{-1 / 2} \overline{\mathbb{L}}(u)\right\} W(t-h u) d u .
$$

and obtain

$$
\begin{aligned}
\left|D_{n, 2}(t)-D_{n, 3}(t)\right| \leq & h^{-1 / 2} \int\left\{h^{\beta} \overline{\mathbb{K}}(u)-\mathcal{K}(u)\right\} f_{X}(t-h u)^{1 / 2} W(t-h u) d u \\
& +h^{1 / 2} \int h^{\beta} \overline{\mathbb{L}}(u) f_{X}^{\prime}(t-h u) f_{X}(t-h u)^{-1 / 2} W(t-h u) d u \\
= & T_{n, 4}(t)+T_{n, 5}(t)
\end{aligned}
$$

Now by the law of the iterated logarithm and Assumption G (ii) and (iv), it follows $\sup _{t \in \mathcal{T}}\left|T_{n, 4}(t)\right|=$ $O_{p}\left(h^{\delta}\right)$. For the term $T_{n, 5}(t)$,

$$
\begin{aligned}
\left|T_{n, 5}(t)\right| \leq & h^{-1 / 2} \sup _{u}\left|h^{\beta} \overline{\mathbb{L}}(u / h)-\mathcal{L}(u / h)\right| \int\left|f_{X}^{\prime}(t-z) f_{X}(t-z)^{-1 / 2} W(t-z)\right| d z \\
& +h^{1 / 2}\left|\int \mathcal{L}(u) f_{X}^{\prime}(t-h u) f_{X}(t-h u)^{-1 / 2} W(t-h u) d u\right| \\
= & T_{n, 51}(t)+T_{n, 52}(t) .
\end{aligned}
$$

Using Assumption G (iii)-(iv), an application of the law of the iterated logarithm proves $\sup _{t \in \mathcal{T}} T_{n, 51}(t)=$ $O\left(h^{\beta}\right)$. Next, for the term $T_{n, 52}(t)$, Hölder's inequality and the law of the iterated logarithm imply

$$
T_{n, 52}(t) \leq h^{\delta_{1} /\left(4-2 \delta_{1}\right)}\|\mathcal{L}(u)\|_{2-\delta_{1}}\left\|f_{X}^{\prime}(u) f_{X}(u)^{-1 / 2} \sqrt{|u| \log \left(\log ^{+}|u|\right)}\right\|_{2+\delta_{1} /\left(1-\delta_{1}\right)} .
$$

By this expression and Assumption G (iii)-(iv), we are able to show $\sup _{t \in \mathcal{T}}\left|T_{n, 52}(t)\right|=o_{p}\left((-\log (h))^{-1 / 2}\right)$. Combining these results, the claim $\sup _{t \in \mathcal{T}}\left|D_{n, 2}(t)-D_{n, 3}(t)\right|=o_{p}\left((-\log (h))^{-1 / 2}\right)$ follows.

Third, we approximate the process $f_{X}(t)^{-1 / 2} D_{n, 3}(t)$ with the process

$$
D_{n, 4}(t)=h^{-3 / 2} \int \mathcal{K}\left(\frac{t-a}{h}\right) W(a) d a .
$$

Note that

$$
f_{X}(t)^{-1 / 2} D_{n, 3}(t)-D_{n, 4}(t)=h^{-1 / 2} \int\left\{f_{X}(t)^{-1 / 2} f_{X}(t-h u)^{1 / 2}-1\right\} \mathcal{K}(u) W(t-h u) d u .
$$

By the law of the iterated logarithm and Assumption G (ii) and (iv), it follows

$$
\sup _{t \in \mathcal{T}}\left|f_{X}(t)^{-1 / 2} D_{n, 3}(t)-D_{n, 4}(t)\right|=O_{p}\left(h^{1 / 2}\right) .
$$

Fourth, let

$$
D_{n, 5}(t)=h^{-1 / 2} \int \mathcal{L}\left(\frac{t-a}{h}\right) d W(a)
$$


By stochastic integration by parts formula and Assumption G (ii),

$$
D_{n, 4}(t)-D_{n, 5}(t)=\left\{\lim _{a \rightarrow \infty} L\left(\frac{t-a}{h}\right) W(a)\right\}-\left\{\lim _{a \rightarrow-\infty} L\left(\frac{t-a}{h}\right) W(a)\right\}=0,
$$

for each $h$, which implies that $D_{n, 4}(t)=D_{n, 5}(t)$ for all $t \in \mathcal{T}$. Since $D_{n, 5}(t)$ has the same finite dimensional distributions as the process $\mathcal{Y}_{n}$, the claim in (C.3) follows. 


\section{REFERENCES}

[1] Barrett, G. F. and S. G. Donald (2003) Consistent tests for stochastic dominance, Econometrica, 71, 71-104.

[2] Bassett, G. W. and R. Koenker (1986) Strong consistency of regression quantiles and related empirical processes, Econometric Theory, 2, 191-201.

[3] Bickel, P. J. and M. Rosenblatt (1973) On some global measures of the deviations of density function estimates, Annals of Statistics, 1, 1071-1095.

[4] Billingsley, P. (1995) Probability and Measure, 3rd edition, Wiley-Blackwell.

[5] Birke, M., Bissantz, N. and H. Holzmann (2010) Confidence bands for inverse regression models, Inverse Problems, 26, 115020.

[6] Bissantz, N., Dümbgen, L., Holzmann, H. and A. Munk (2007) Non-parametric confidence bands in deconvolution density estimation, Journal of the Royal Statistical Society, B 69, 483-506.

[7] Bonhomme, S. and J.-M. Robin (2010) Generalized non-parametric deconvolution with an application to earnings dynamics, Review of Economic Studies, 77, 491-533.

[8] Bound, J., Brown, C. and N. Mathiowetz (2000) Measurement error in survey data, Handbook of Econometrics, vol. 5, chapter 59, Elsevier.

[9] Bound, J. and A. B. Krueger (1991) The extent of measurement error in longitudinal earnings data: Do two wrongs make a right?, Journal of Labor Economics, 9, 1-24.

[10] Chernozhukov, V., Chetverikov, D. and K. Kato (2014) Anti-concentration and honest, adaptive confidence bands, Annals of Statistics, 42, 1787-1818.

[11] Chernozhukov, V., Chetverikov, D. and K. Kato (2015) Comparison and anti-concentration bounds for maxima of Gaussian random vectors, Probability Theory and Related Fields, 162, 47-70.

[12] Comte, F. and J. Kappus (2015) Density deconvolution from repeated measurement without symmetry assumption on the errors, Journal of Multivariate Analysis, 140, 31-46.

[13] Dattner, I., Goldenshluger, A. and A. Juditsky (2011) On deconvolution of distribution functions, Annals of Statistics, 39, 2477-2501.

[14] Dattner, I., Reiß, M. and M. Trabs (2016) Adaptive quantile estimation in deconvolution with unknown error distribution, Bernoulli, 22, 143-192.

[15] Deaton, A. (1997) The Analysis of Household Surveys: A Microeconometric Approach to Development Policy, Baltimore: Johns Hopkins University Press for the World Bank.

[16] Delaigle, A. and I. Gijbels (2004) Bootstrap bandwidth selection in kernel density estimation from a contaminated sample, Annals of the Institute of Statistical Mathematics, 56, 19-47.

[17] Delaigle, A. and P. Hall (2006) On optimal kernel choice for deconvolution, Statistics 8 Probability Letters, $76,1594-1602$.

[18] Delaigle, A., Hall, P. and F. Jamshidi (2015) Confidence bands in nonparametric errors-in- variables regression, Journal of the Royal Statistical Society, B 77, 149-169.

[19] Delaigle, A., Hall, P. and A. Meister (2008) On deconvolution with repeated measurements, Annals of Statistics, 36, 665-685.

[20] Efromovich, S. (1997) Density estimation for the case of supersmooth measurement error, Journal of the American Statistical Association, 92, 526-535.

[21] Fan, J. (1991) On the optimal rates of convergence for nonparametric deconvolution problems, Annals of Statistics, 19, 1257-1272. 
[22] Giné, E. and R. Nickl (2016) Mathematical Foundations of Infinite-Dimensional Statistical Models, Cambridge University Press.

[23] Hall, P. and S. N. Lahiri (2008) Estimation of distributions, moments and quantiles in deconvolution problems, Annals of Statistics, 36, 2110-2134.

[24] Hall, P. and A. Meister (2007) A ridge-parameter approach to deconvolution, Annals of Statistics, 35, 15351558.

[25] Hu and Sasaki (2015) Closed-form estimation of nonparametric models with non-classical measurement errors, Journal of Econometrics, 185, 392-408

[26] Kato, K. and Y. Sasaki (2018) Uniform confidence bands in deconvolution with unknown error distribution, Journal of Econometrics, 207, 129-161.

[27] Kato, K. and Y. Sasaki (2017) Uniform confidence bands for nonparametric errors-in-variables regression, Working paper.

[28] Kato, K., Sasaki, Y. and T. Ura (2019) Inference based on Kotlarski's identity, Working paper.

[29] Komlós, J., Major, P. and G. Tusnády (1975) An approximation of partial sums of independent RV'-s and the sample DF. I, Zeitschrift fur Wahrscheinlichkeitstheorie und verwandte Gebiete, 32, 111-131.

[30] Kurisu, D. and T. Otsu (2019) On the uniform convergence of deconvolution estimators from repeated measurements, Working paper.

[31] Lepski, O. V. (1990) A problem of adaptive estimation in Gaussian white noise, Teor. Veroyatnost. $i$ Primenen., 35, 459-470.

[32] Levy, H. (2016) Stochastic Dominance, Springer.

[33] Li, T. and Q. Vuong (1998) Nonparametric estimation of the measurement error model using multiple indicators, Journal of Multivariate Analysis, 65, 139-165.

[34] Meister, A. (2009) Deconvolution Problems in Nonparametric Statistics, Springer.

[35] Neumann, M.H (1997) On the effect of estimating the error density in nonparametric deconvolution, Journal of Nonparametric Statistics, 7, 307-330.

[36] OECD (2008) Growing unequal? Income distribution and poverty in OECD countries, Paris: OECD.

[37] Proksh, K., Bissantz, N. and H. Dette (2015) Confidence bands for multivariate and time dependent inverse regression models, Bernoulli, 21, 144-175.

[38] Schennach, S. M. (2004) Nonparametric regression in the presence of measurement error, Econometric Theory, 10, 1046-1093

[39] Schennach, S. M. (2013) Convolution without independence, Working paper.

[40] Shorack, G. R. (1982) Bootstrapping robust regression, Communications in Statistics: Theory and Methods, $11,961-972$.

[41] Söhl, J. and M. Trabs (2012) A uniform central limit theorem and efficiency for deconvolution estimators, Electronic Journal of Statistics, 6, 2486-2518.

[42] Stefanski, L. and Carroll, R. J. (1990) Deconvoluting kernel density estimators, Statistics, 21, 169-184.

[43] van der Vaart, A. W. and J. Wellner (1996) Weak Convergence and Empirical Processes, Springer.

[44] van Es, A. J. and H.-W. Uh (2005) Asymptotic normality for kernel type deconvolution estimators, Scandinavian Journal of Statistics, 32, 467-483.

[45] Whang, Y.-J. (2018) Econometric Analysis of Stochastic Dominance, Cambridge University Press.

[46] Yukich, J. E. (1987) Some limit theorems for the empirical process indexed by functions, Probability Theory and Related Fields, 74, 71-90. 
Department of Economics, University of Pennsylvania, 133 South 36th Street, Philadelphia, PA 19104, USA.

E-mail address: k.adusumilli@lse.ac.uk

Department of Industrial Engineering and Economics, School of Engineering, Tokyo Institute of Technology, 2-12-1 Ookayama, Meguro-ku, Tokyo 152-8552, Japan.

E-mail address: kurisu.d.aa@m.titech.ac.jp

Department of Economics, London School of Economics, Houghton Street, London, WC2A 2AE, UK.

E-mail address: t.otsu@lse.ac.uk

Department of Economics, Seoul National University, 1 Gwankro Gwanakgu, Seoul, 08826, KoREA.

E-mail address: whang@snu.ac.kr 